\title{
WHITE DWARF-RED DWARF SYSTEMS RESOLVED WITH THE HUBBLE SPACE TELESCOPE. II. FULL SNAPSHOT SURVEY RESULTS
}

\author{
J. FARIHI ${ }^{1}$, D. W. HOARD ${ }^{2}$, AND S. WACHTER ${ }^{2}$ \\ ${ }^{1}$ Department of Physics \& Astronomy, University of Leicester, Leicester LE1 7RH, UK; jf123@star.le.ac.uk \\ ${ }^{2}$ Spitzer Science Center, California Institute of Technology, MS 220-6, Pasadena, CA 91125, USA \\ Received 2010 June 8; accepted 2010 August 12; published 2010 September 17
}

\begin{abstract}
Results are presented for a Hubble Space Telescope Advanced Camera for Surveys high-resolution imaging campaign of 90 white dwarfs with known or suspected low-mass stellar and substellar companions. Of the 72 targets that remain candidate and confirmed white dwarfs with near-infrared excess, 43 are spatially resolved into two or more components, and a total of 12 systems are potentially triples. For 68 systems where a comparison is possible, 50\% have significant photometric distance mismatches between their white dwarf and $\mathrm{M}$ dwarf components, suggesting that white dwarf parameters derived spectroscopically are often biased due to the cool companion. Interestingly, 9 of the 30 binaries known to have emission lines are found to be visual pairs and hence widely separated, indicating an intrinsically active cool star and not irradiation from the white dwarf. There is a possible, slight deficit of earlier spectral types (bluer colors) among the spatially unresolved companions, exactly the opposite of expectations if significant mass is transferred to the companion during the common envelope phase. Using the best available distance estimates, the low-mass companions to white dwarfs exhibit a bimodal distribution in projected separation. This result supports the hypothesis that during the giant phases of the white dwarf progenitor, any unevolved companions either migrate inward to short periods of hours to days, or outward to periods of hundreds to thousands of years. No intermediate projected separations of a few to several $\mathrm{AU}$ are found among these pairs. However, a few double M dwarfs (within triples) are spatially resolved in this range, empirically demonstrating that such separations were readily detectable among the binaries with white dwarfs. A straightforward and testable prediction emerges: all spatially unresolved, low-mass stellar and substellar companions to white dwarfs should be in short-period orbits. This result has implications for substellar companion and planetary orbital evolution during the post-main-sequence lifetime of their stellar hosts.
\end{abstract}

Key words: binaries: general - brown dwarfs - stars: evolution - stars: formation - stars: low-mass - stars: luminosity function, mass function - white dwarfs

\section{INTRODUCTION}

Unlike main-sequence stars, white dwarfs allow one to see more deeply into their local spatial environments due to low (and blue-peaked) luminosities; quite possibly providing an excellent window to directly detect extrasolar planets (Hogan et al. 2009; Burleigh et al. 2002). While surviving planets orbiting white dwarfs probably exist in modest abundance (Farihi et al. 2009), and their post-main-sequence evolution is of obvious interest, they are currently difficult to detect. Substellar companions to white dwarfs are the best proxy for any giant planets that survive beyond the main sequence, but white dwarf-brown dwarf binaries are rare (Farihi et al. 2008, 2005a; Mullally et al. 2007; Hoard et al. 2007). Low-mass stellar companions to white dwarfs, however, are relatively ubiquitous and produce a composite color signature that is readily detectable (Hoard et al. 2007; Silvestri et al. 2006; Probst 1983). These binary systems can provide basic information on the long-term evolution of lowmass objects in the presence of host giant stellar envelopes, and the initial mass function near the bottom of the main sequence (Schultz et al. 1996; Zuckerman \& Becklin 1992).

Understanding common envelope evolution is critical for additional astrophysical phenomena such as planetary nebulae, cataclysmic variables, $\mathrm{X}$-ray binaries, and compact stellar mergers. The latter are perhaps the best candidates for Type Ia supernovae and short $\gamma$-ray bursts (De Marco 2009; Tappert et al. 2009; Nelemans et al. 2005). Here, low-mass stellar companions to white dwarfs are used to constrain the effects of common envelope evolution by directly measuring the distribution of their projected separations and secondary star colors; these quantities are excellent indicators of orbital semimajor axes and masses. This is a fundamental astrophysical question: how does postmain-sequence evolution influence the distribution of binary parameters?

This paper presents astrometric and photometric data for 90 white dwarfs with suspected near-infrared excess owing to a low-mass stellar or substellar companion. The program, data acquisition, and analysis are described in Section 2, and the results are discussed in Section 3. The first half of this data set was presented in Farihi et al. (2006, Paper I), but the results given here, which were produced using a uniform application of a more recent processing pipeline, supersede those in Paper I.

\section{THE PROGRAM AND OBSERVATIONAL DATA}

The observational goal of the program was to image white dwarfs with low-mass, cool companions at sufficient spatial resolution to directly detect any secondaries in the few to several AU range; i.e., orbital periods of one to tens of years which are difficult to probe from the ground. The targets were chosen from ground-based data sets as confirmed or candidate, spatially unresolved binaries consisting of a known or suspected white dwarf with near-infrared excess emission (Hoard et al. 2007; Farihi et al. 2005a; Wachter et al. 2003; McCook \& Sion 1999).

In Cycle 13, Hubble Space Telescope (HST) Snapshot Program 10255 was awarded 100 targets for single bandpass imaging with the Advanced Camera for Surveys (ACS; Ford et al. 1998) High-Resolution Camera (HRC). The F814W filter was 
chosen as the most likely wavelength range in which both the white dwarf and low-mass components would be relatively bright, and would still be sensitive to the latest $\mathrm{M}$ dwarf companions. The images were acquired in a four-point dither pattern with individual $10 \mathrm{~s}$ exposures for all but two stars that had suspected or known brown dwarf companions; for those targets the exposures were $270 \mathrm{~s}$ each. The data for each target were processed into a single, multi-drizzled image with the ACS calibration software and pipeline CALACS 4.5.6 and OPUS 15.7; this represents a more recent pipeline than that used for the subset of observations analyzed for Paper I and may account for some of the minor numerical differences (see descriptions of individual targets in the Appendix).

Figures 1(a)-(c) display the ACS images of 17 newly detected multiple systems with spatially resolved components. Images of the remaining 28 systems with components that were spatially resolved in the ACS program are shown in Paper I. All other science target images were found to be consistent with point sources.

\subsection{Photometry and Astrometry}

All flux measurements were carried out using an aperture radius of $r=0$ '.20 (8 pixels; includes first Airy maximum and $85 \%$ of energy), with standard sky annulus, aperture corrections, photometric transformations following Sirianni et al. (2005), and Vega zero points from Bohlin (2007). Photometry was performed with the IRAF $^{3}$ task apphot for all point sources isolated by a radius of at least 0 '. $^{\prime}$. For pairs more closely separated than this, daophot was used to simultaneously photometrically deconvolve the sources. A total of 12 point spread function (PSF) stars were chosen among the highest signal-to-noise ratio $(\mathrm{S} / \mathrm{N})$, isolated point sources; four of these were white dwarfs, four were $\mathrm{M}$ dwarfs, and four were spatially unresolved white dwarf-M dwarf pairs. For the 22 close visual doubles and triples, the components were fitted with each of the 12 PSF stars, and photometry was determined by averaging across values within the appropriate subtype. In a few cases, only one or two PSF stars produced consistent results with acceptable errors, and other fits were rejected. Photometric errors are the quadrature sum of the measurement errors via apphot or daophot and a $2 \%$ calibration error; the Cousins $I$-band magnitude errors include an additional uncertainty from the transformation equations (Sirianni et al. 2005).

Astrometry was performed with IDP $3^{4}$ radial profile fitting at $r=3$ pixels. This radius is an ideal choice for image centroiding and PSF characterization as it occurs near the first Airy pattern minimum, and interior to this radius the PSF is well reproduced by a Gaussian radial profile. For isolated sources, Gaussian fit centroid errors were typically less than 0.03 pixels along both the $x$ - and $y$-axes. Close visual pairs had centroid errors somewhat larger and determined by the scatter in positions from multiple PSF fits and/or images in which the neighboring source was fitted and subtracted; where multiple methods were used, a weighted average was taken. The errors in binary angular separation and position angle were calculated by direct propagation of measured $x$ - and $y$-axis positional errors through the formulae. Full widths at half-maximum (FWHMs) were measured using Gaussian fits of the radial profile out

\footnotetext{
3 IRAF is distributed by the National Optical Astronomy Observatory, which is operated by the Association of Universities for Research in Astronomy, Inc., under cooperative agreement with the National Science Foundation.

4 http://mips.as.arizona.edu/MIPS/IDP3
}

to $r=3$ pixels, corresponding to a diameter of two typical FWHMs.

The size of the F814W aperture correction and subsequent photometric transformation to Cousins $I$ band are both color dependent, and were determined using Tables 7, 8, and 23 of Sirianni et al. (2005). First, the published or best estimate of the $V-I$ (for white dwarfs) or $I-K$ (for $\mathrm{M}$ dwarfs) color of a given star was used to look up a color-dependent aperture correction, utilizing any available colors or spectral type estimate. Second, a Cousins I magnitude was calculated on the basis of this estimated color and the aperture-corrected F814W magnitude. Third, this $I$-band magnitude was used to re-determine $V-I$ or $I-K$, and the two previous steps were repeated until consistent results were achieved. This process required three or fewer iterations, and a typical error is 0.02 mag or less. For spatially unresolved binaries, the above process was repeated for each component until the flux ratio and the composite color were consistent (see Section 2.2).

All astrometric and photometric data are listed in Table 1. The first and second columns list the white dwarf designations (McCook \& Sion 1999) and common names of all 90 targets. The third column indicates if the target is spatially resolved into multiple components. The fourth and fifth columns list the FWHM of the stellar PSF and the ratio of the semimajor to semiminor axis $(a / b)$ of the radial profile fit. For close visual pairs, the sixth and seventh columns list the angular separation and position angle of the secondary star. The eighth and ninth columns give the F814W and Cousins $I$-band photometry for all single and composite point sources. The final column contains information on the number and nature of the imaged sources. Table 2 lists the coordinates of all primary (white dwarf) targets within the ACS images, some of which, as noted in the table, have erroneous or inaccurate values in SIMBAD and the literature.

\subsection{Binary and Multiple Component Analysis}

Component identification for spatially resolved multiples imaged in a single filter is difficult, but fortunately the ACS F814W PSF contains color information (Sirianni et al. 2005). Figure 2 plots the applied, color-dependent, aperture correction (i.e., the expected $V-I$ color based on the best spectral type or temperature estimate) versus the measured FWHM. The isolated white dwarfs occupy the region blueward of $0.28 \mathrm{mag}$, and the isolated low-mass stars all sit redward of 0.30 mag, while the region between represents composite systems. While the FWHMs for different stellar types somewhat overlap, there is a clear trend as well as a number of outliers, some of which are certain or suspected binaries.

Combining the well-understood ACS PSF color trend (Sirianni et al. 2005) with the expected $I$-band brightness for the components (based on photometric catalogs and the literature) yields an unambiguous identification of the white dwarf and cool companion within all the ACS imaged, spatially resolved binaries. First, published ultraviolet-blue and near-infrared data for each pair provide a broad but good indication of the expected brightness for both the relatively hot and cool stars in the F814W filter. Only in a single case were the brightnesses sufficiently close for identification to remain uncertain. Second, for all spatially well-resolved binaries where the recognition of the white dwarf and M dwarf components is solid, the FWHM of the M dwarf is larger than that of the white dwarf by an average of 0.1 pixel (a single exception is a suspected unresolved binary). 


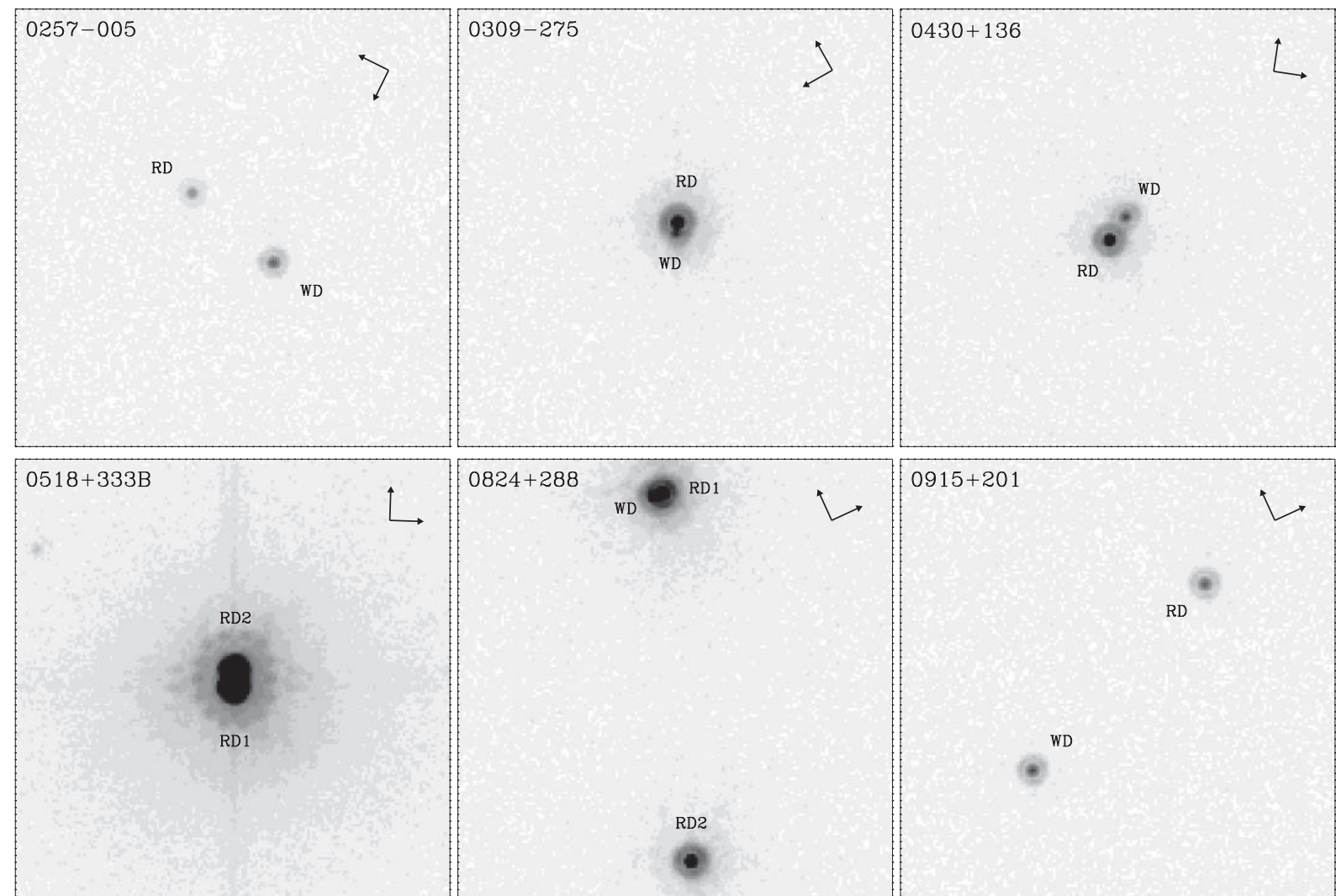

(a)

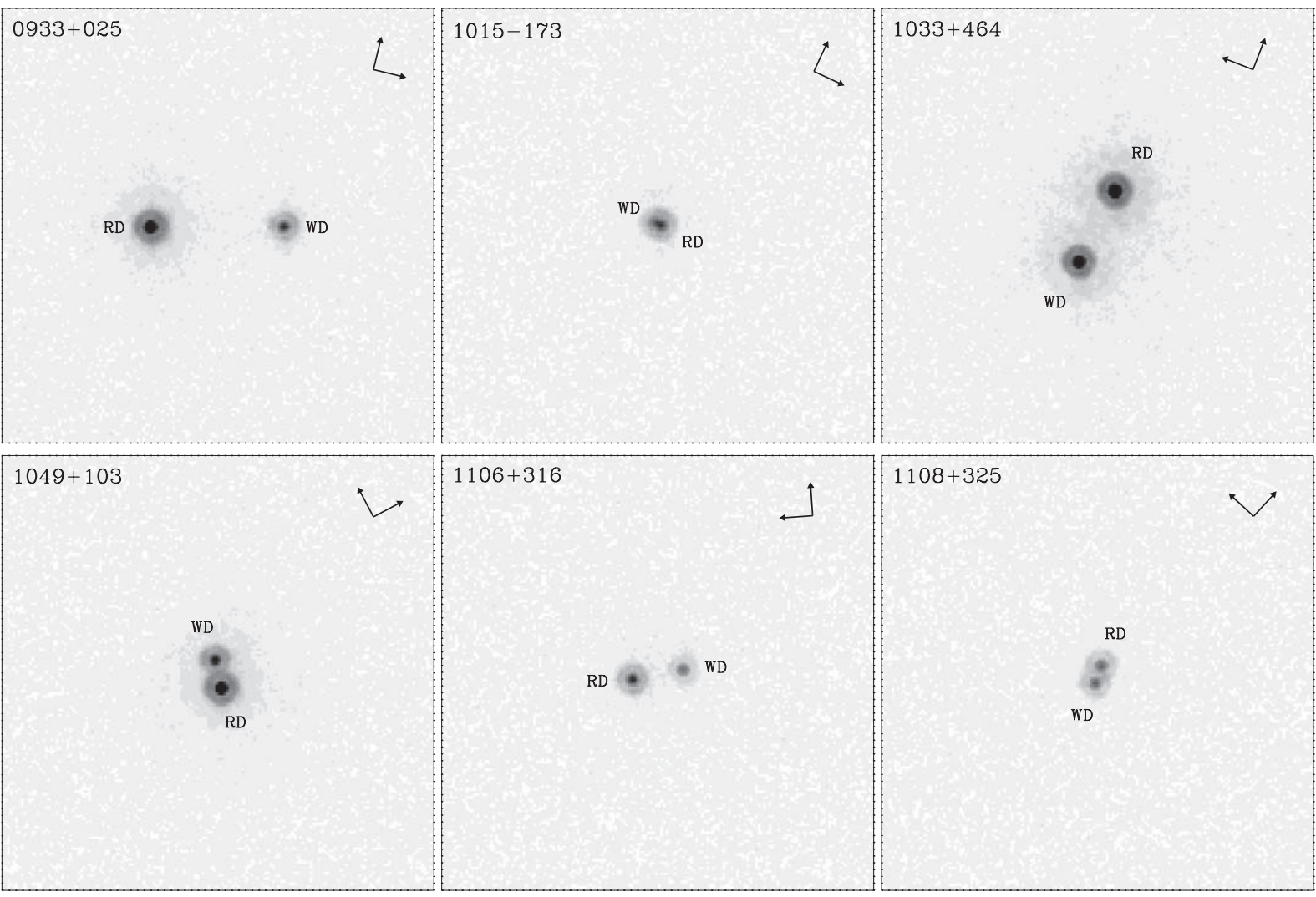

(b)

Figure 1. Multidrizzled, ACS HRC images of all 17 newly detected, totally or partially resolved, multiple systems. All data were taken in the F814W filter and are displayed at a single color scale and stretch. The panels are $4.0 \times 44^{\prime \prime} 0$ and displayed in the native spacecraft orientation with arrows indicating the directions of north and east. 


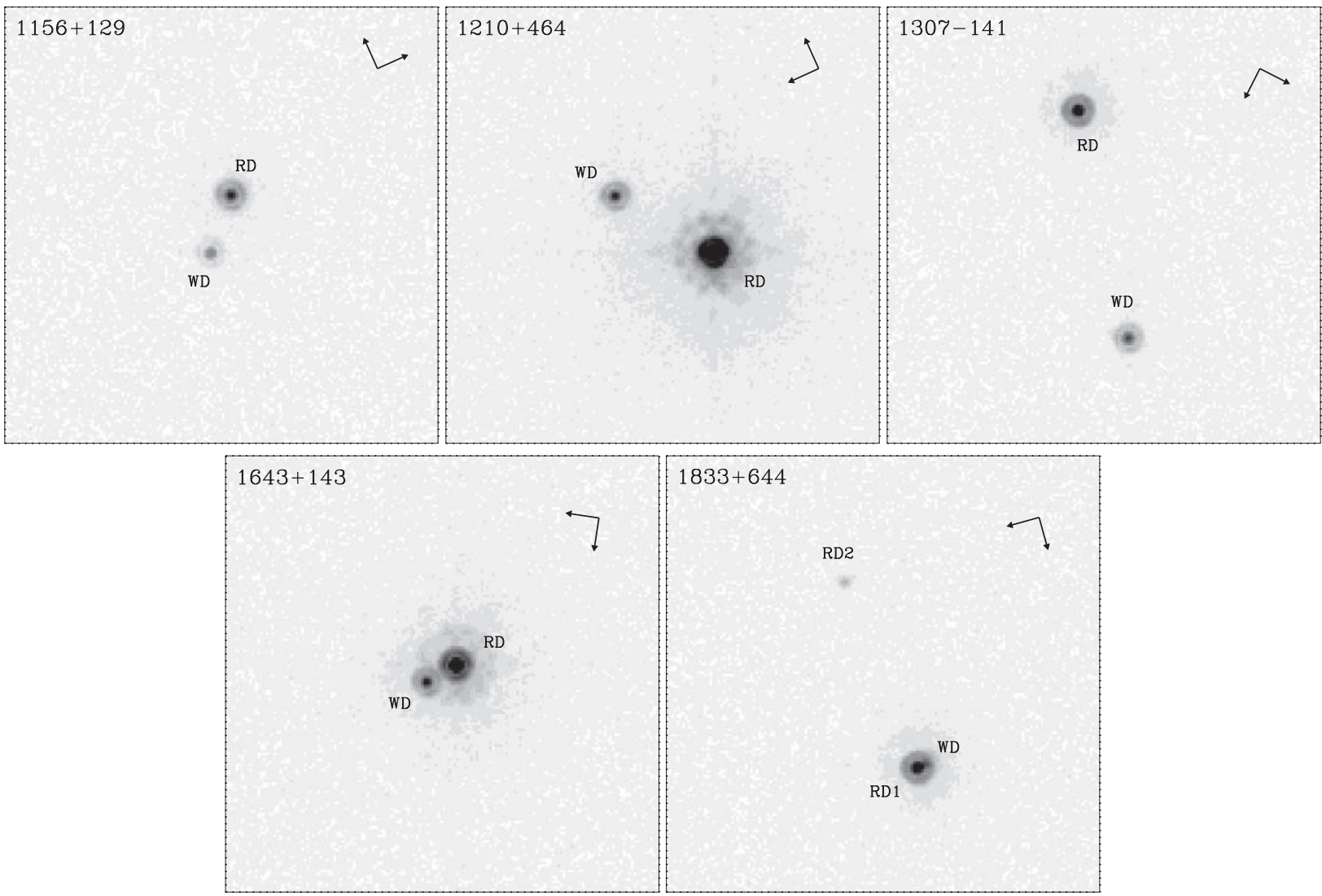

(c)

Figure 1. (Continued)

For each white dwarf, the literature was scoured for available optical photometry (Abazajian et al. 2009; Copenhagen University Observatory 2006; DENIS Consortium 2005), effective temperature, and surface gravity determinations (McCook \& Sion 2008, 1999). Near-infrared photometry was taken from the Two Micron All Sky Survey (2MASS; Skrutskie et al. 2006), while far- and near-ultraviolet fluxes were taken from archival Galaxy Evolution Explorer (GALEX; Martin et al. 2005) data where available. The GALEX fluxes were used to constrain the effective temperature in systems lacking a reliable estimate, via comparison with ultraviolet-optical flux ratios for stars of known temperature. In ideal cases, Sloan Digital Sky Survey (SDSS) $u g$-band or Johnson $U B$-band photometry could be combined with models to predict the white dwarf flux at longer wavelengths. Generally, any photometric data unlikely to be contaminated by the cool companion were utilized to predict the white dwarf brightness at VIJHK. For those systems in which the white dwarf was spatially resolved from its secondary, the model and measured $I$-band magnitude could be directly compared.

Table 3 lists the adopted white dwarf parameters. All model effective temperatures, surface gravities, masses, absolute magnitudes, and colors were taken from Holberg \& Bergeron (2006) and Fontaine et al. (2001). Where a reliable surface gravity determination is lacking, $0.6 M_{\odot}$ was assumed, equivalent to $\log \left[g\left(\mathrm{~cm} \mathrm{~s}^{-2}\right)\right] \approx 8$ and represented by single decimal place precision in the third column. The $V$-band magnitudes in the fourth column are values predicted for the white dwarf alone, based on model colors and photometry in bandpasses that are certainly or unlikely to be contaminated by the $\mathrm{M}$ dwarf. The photometric distances to the white dwarfs were derived from $V-M_{V}$.

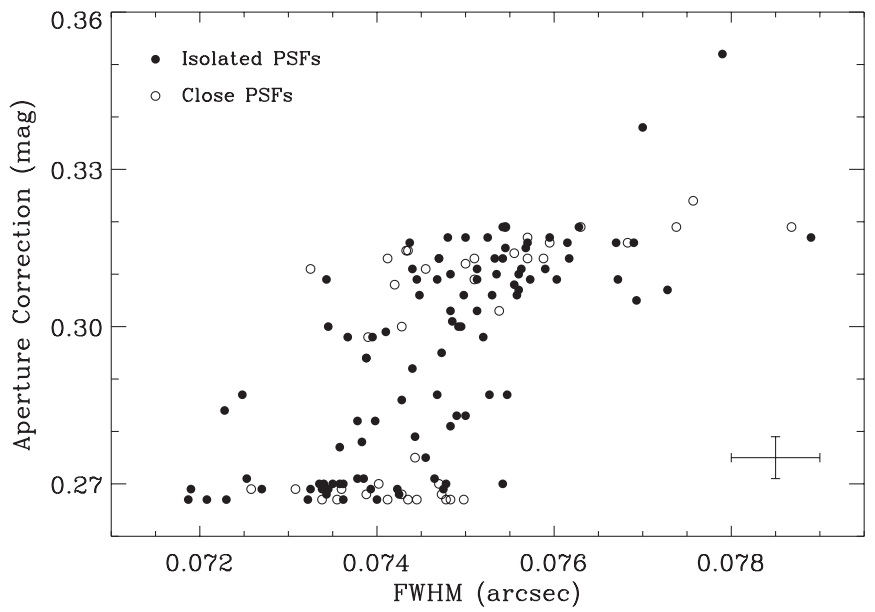

Figure 2. The adopted, color-dependent aperture corrections in F814W Vega magnitudes for all 141 directly imaged white dwarf and companion point sources, plotted vs. the measured FWHM. This correction is a reflection of the best available estimate of the color (spectral type) of each star, and is sometimes uncertain where little independent data exist. The error bars at the lower right display a \pm 0.02 pixel uncertainty in FWHM measurements, and an uncertainty in aperture correction corresponding to \pm 1 spectral type at M4, with bluer objects having smaller errors than this benchmark. The filled circles represent stellar images that are well isolated from any companions, while the open circles are plotted for sources with companions within 0!'4 (i.e., the two photometric apertures overlap).

For spatially unresolved white dwarf-M dwarf pairs, the IJHK magnitudes of the $\mathrm{M}$ dwarf component were calculated by subtracting the expected white dwarf flux in those bandpasses from the composite photometry. If the $\mathrm{M}$ dwarf was spatially resolved from the white dwarf, its $I$-band magnitude was 
Table 1

Target ACS Photometric and Astrometric Data

\begin{tabular}{|c|c|c|c|c|c|c|c|c|c|}
\hline WD & Name & $\begin{array}{c}\text { Spatially } \\
\text { Resolved? }\end{array}$ & $\begin{array}{l}\text { FWHM } \\
(\operatorname{arcsec})\end{array}$ & $a / b$ & $\begin{array}{c}\alpha \\
(\operatorname{arcsec})\end{array}$ & $\begin{array}{l}\text { P.A. } \\
\text { (deg) }\end{array}$ & $\begin{array}{c}\mathrm{F}^{8} 14 \mathrm{~W}^{\mathrm{a}} \\
(\mathrm{mag})\end{array}$ & $\begin{array}{c}I_{c} \\
(\mathrm{mag})\end{array}$ & $\begin{array}{c}\text { Source } \\
\text { Notes } \\
\end{array}$ \\
\hline $0023+388$ & G171-B10A & No & 0.07367 & 1.0294 & $\ldots$ & $\ldots$ & $15.22 \pm 0.02$ & $15.22 \pm 0.03$ & 1 \\
\hline \multirow[t]{2}{*}{$0034-211$} & LTT 0329 & Yes & 0.07470 & 1.0350 & $\ldots$ & $\ldots$ & $15.11 \pm 0.02$ & $15.07 \pm 0.03$ & 2,5 \\
\hline & & & 0.07412 & 1.0222 & $0.327 \pm 0.001$ & $105.25 \pm 0.09$ & $12.76 \pm 0.02$ & $12.81 \pm 0.03$ & 5 \\
\hline \multirow[t]{2}{*}{$0116-231$} & GD 695 & Yes & 0.07393 & 1.0071 & $\ldots$ & $\ldots$ & $16.59 \pm 0.02$ & $16.54 \pm 0.03$ & 2 \\
\hline & & & 0.07570 & 1.0211 & $1.106 \pm 0.001$ & $9.95 \pm 0.05$ & $16.15 \pm 0.02$ & $16.23 \pm 0.03$ & \\
\hline \multirow[t]{2}{*}{$0131-163$} & GD 984 & Yes & 0.07412 & 1.0147 & $\ldots$ & $\ldots$ & $14.29 \pm 0.02$ & $14.23 \pm 0.03$ & 2,5 \\
\hline & & & 0.07435 & 1.0186 & $0.190 \pm 0.001$ & $145.34 \pm 0.17$ & $14.50 \pm 0.02$ & $14.57 \pm 0.03$ & 5 \\
\hline $0145-221$ & GD 1400 & No & 0.07358 & 1.0025 & $\ldots$ & $\ldots$ & $15.16 \pm 0.02$ & $15.13 \pm 0.03$ & 1 \\
\hline \multirow[t]{2}{*}{$0145-257$} & GD 1401 & Yes & 0.07338 & 1.0169 & $\ldots$ & $\ldots$ & $14.97 \pm 0.02$ & $14.93 \pm 0.03$ & 2 \\
\hline & & & 0.07568 & 1.0103 & $2.294 \pm 0.001$ & $154.06 \pm 0.01$ & $13.83 \pm 0.02$ & $13.90 \pm 0.03$ & \\
\hline \multirow[t]{2}{*}{$0205+133$} & PG & Yes & 0.07362 & 1.0433 & $\ldots$ & $\ldots$ & $15.45 \pm 0.02$ & $15.40 \pm 0.03$ & 2 \\
\hline & & & 0.07573 & 1.0360 & $1.254 \pm 0.001$ & $10.60 \pm 0.02$ & $13.94 \pm 0.02$ & $13.97 \pm 0.03$ & \\
\hline \multirow[t]{2}{*}{$0208-153$} & МСТ $0208-1520$ & Yes & 0.07362 & 1.0211 & $\ldots$ & $\ldots$ & $15.94 \pm 0.02$ & $15.90 \pm 0.03$ & 2 \\
\hline & & & 0.07563 & 1.0194 & $2.648 \pm 0.001$ & $217.43 \pm 0.02$ & $13.80 \pm 0.02$ & $13.85 \pm 0.03$ & \\
\hline \multirow[t]{2}{*}{$0219+282$} & KUV 02196+2816 & Yes & 0.07473 & 1.0089 & $\ldots$ & $\ldots$ & $17.33 \pm 0.03$ & $17.28 \pm 0.04$ & 2,5 \\
\hline & & & 0.07868 & 1.0310 & $0.116 \pm 0.002$ & $193.37 \pm 0.19$ & $18.14 \pm 0.04$ & $18.26 \pm 0.05$ & 5 \\
\hline \multirow[t]{2}{*}{$0237+115$} & PG & Yes & 0.07338 & 1.0240 & $\ldots$ & $\ldots$ & $16.38 \pm 0.02$ & $16.32 \pm 0.03$ & 2,5 \\
\hline & & & 0.07455 & 1.0098 & $0.124 \pm 0.002$ & $268.52 \pm 0.21$ & $15.00 \pm 0.02$ & $15.05 \pm 0.03$ & 5 \\
\hline \multirow[t]{2}{*}{$0257-005$} & KUV 02579-0036 & Yes & 0.07230 & 1.0233 & $\ldots$ & $\ldots$ & $17.37 \pm 0.03$ & $17.32 \pm 0.04$ & 2 \\
\hline & & & 0.07525 & 1.0501 & $0.978 \pm 0.002$ & $112.99 \pm 0.15$ & $18.61 \pm 0.05$ & $18.70 \pm 0.06$ & \\
\hline $0303-007$ & KUV 03036-0043 & No & 0.07535 & 1.0232 & $\ldots$ & $\ldots$ & $14.54 \pm 0.02$ & $14.59 \pm 0.03$ & 1 \\
\hline \multirow[t]{2}{*}{$0309-275$} & & Yes & 0.07498 & 1.0359 & $\ldots$ & $\ldots$ & $16.84 \pm 0.04$ & $16.78 \pm 0.05$ & 2,5 \\
\hline & & & 0.07538 & 1.0076 & $0.099 \pm 0.002$ & $18.32 \pm 0.43$ & $14.59 \pm 0.02$ & $14.61 \pm 0.03$ & 5 \\
\hline \multirow[t]{3}{*}{$0324+738$} & $\mathrm{G} 221-10$ & No & 0.07398 & 1.0435 & $\ldots$ & $\ldots$ & $16.55 \pm 0.02$ & $16.53 \pm 0.03$ & 1,7 \\
\hline & G221-11 & Yes & 0.07545 & 1.0322 & $\ldots$ & $\ldots$ & $13.69 \pm 0.02$ & $13.82 \pm 0.03$ & $2,5,6$ \\
\hline & & & 0.07757 & 1.0322 & $0.297 \pm 0.001$ & $31.84 \pm 0.05$ & $15.11 \pm 0.02$ & $15.30 \pm 0.03$ & 5 \\
\hline $0331-356$ & HE $0331-3541$ & No & 0.07590 & 1.0271 & $\ldots$ & $\ldots$ & $12.72 \pm 0.02$ & $12.77 \pm 0.03$ & 1 \\
\hline \multirow[t]{2}{*}{$0347-137$} & GD 51 & Yes & 0.07378 & 1.0168 & $\ldots$ & $\ldots$ & $15.50 \pm 0.02$ & $15.46 \pm 0.03$ & 2 \\
\hline & & & 0.07690 & 1.0247 & $1.052 \pm 0.001$ & $265.10 \pm 0.03$ & $13.57 \pm 0.02$ & $13.65 \pm 0.03$ & \\
\hline $0354+463$ & Rubin 80 & No & 0.07495 & 1.0181 & $\ldots$ & $\ldots$ & $14.89 \pm 0.02$ & $14.90 \pm 0.03$ & 1 \\
\hline $0357-233$ & Ton S 392 & Yes & 0.07322 & 1.0176 & $\ldots$ & $\ldots$ & $16.24 \pm 0.02$ & $16.18 \pm 0.03$ & 2 \\
\hline & & & 0.07533 & 1.0094 & $1.191 \pm 0.001$ & $350.86 \pm 0.05$ & $16.47 \pm 0.02$ & $16.53 \pm 0.03$ & \\
\hline $0430+136$ & KUV 04304+1339 & Yes & 0.07428 & 1.0408 & $\ldots$ & $\ldots$ & $16.75 \pm 0.03$ & $16.76 \pm 0.04$ & 2,5 \\
\hline & & & 0.07683 & 1.0370 & $0.260 \pm 0.001$ & $47.37 \pm 0.04$ & $15.11 \pm 0.02$ & $15.19 \pm 0.03$ & 5 \\
\hline $0458-665$ & RX J0458.9-6628 & No & 0.07470 & 1.0219 & $\ldots$ & $\ldots$ & $14.56 \pm 0.02$ & $14.61 \pm 0.03$ & 1 \\
\hline $0518+333$ & G86-1B & No & 0.07383 & 1.0229 & $\ldots$ & $\ldots$ & $15.85 \pm 0.02$ & $15.83 \pm 0.03$ & 1,7 \\
\hline & G86-1A & Yes & 0.07500 & 1.0381 & $\ldots$ & $\ldots$ & $12.41 \pm 0.02$ & $12.46 \pm 0.03$ & $2,5,6$ \\
\hline & & & 0.07555 & 1.0341 & $0.158 \pm 0.001$ & $266.31 \pm 0.13$ & $12.77 \pm 0.02$ & $12.83 \pm 0.03$ & 5 \\
\hline $0752-146$ & LTT 2980 & No & 0.07378 & 1.0395 & $\ldots$ & $\ldots$ & $13.47 \pm 0.02$ & $13.44 \pm 0.03$ & 1 \\
\hline $0807+190$ & LHS 1986 & No & 0.07595 & 1.0308 & $\ldots$ & $\ldots$ & $16.74 \pm 0.02$ & $16.84 \pm 0.03$ & 1,7 \\
\hline $0812+478$ & PG & No & 0.07490 & 1.0239 & $\ldots$ & $\ldots$ & $15.15 \pm 0.02$ & $15.13 \pm 0.03$ & 1 \\
\hline $0824+288$ & PG & Yes & 0.07445 & 1.0180 & $\ldots$ & $\ldots$ & $15.12 \pm 0.03$ & $15.07 \pm 0.04$ & 3,5 \\
\hline & & & 0.07390 & 1.0324 & $0.077 \pm 0.002$ & $228.28 \pm 0.45$ & $13.92 \pm 0.02$ & $13.92 \pm 0.03$ & 5 \\
\hline & & & 0.07513 & 1.0418 & $3.330 \pm 0.001$ & $120.56 \pm 0.01$ & $14.47 \pm 0.02$ & $14.51 \pm 0.03$ & \\
\hline $0908+226$ & LP $369-15$ & No & 0.07542 & 1.0386 & $\ldots$ & $\ldots$ & $16.46 \pm 0.02$ & $16.52 \pm 0.03$ & 1 \\
\hline $0915+201$ & LB 3016 & Yes & 0.07187 & 1.0380 & $\ldots$ & $\ldots$ & $16.92 \pm 0.02$ & $16.86 \pm 0.03$ & 2 \\
\hline & & & 0.07470 & 1.0224 & $2.312 \pm 0.002$ & $251.74 \pm 0.04$ & $17.11 \pm 0.03$ & $17.16 \pm 0.04$ & \\
\hline $0933+025$ & PG & Yes & 0.07343 & 1.0529 & $\ldots$ & $\ldots$ & $16.52 \pm 0.02$ & $16.48 \pm 0.03$ & 2 \\
\hline & & & 0.07437 & 1.0499 & $1.232 \pm 0.001$ & $346.60 \pm 0.05$ & $14.74 \pm 0.02$ & $14.82 \pm 0.03$ & \\
\hline 0937-095 & LDS 3913A & No & 0.07388 & 1.0455 & $\ldots$ & $\ldots$ & $14.60 \pm 0.02$ & $14.60 \pm 0.03$ & 1,7 \\
\hline & LDS 3913B & No & 0.07410 & 1.0359 & $\ldots$ & $\ldots$ & $15.63 \pm 0.02$ & $15.63 \pm 0.03$ & 1 \\
\hline $0949+451$ & HS $0949+4508$ & Yes & 0.07455 & 1.0177 & $\ldots$ & $\ldots$ & $15.89 \pm 0.02$ & $15.86 \pm 0.03$ & 2 \\
\hline & & & 0.07890 & 1.1256 & $2.892 \pm 0.001$ & $119.63 \pm 0.01$ & $13.50 \pm 0.02$ & $13.60 \pm 0.03$ & 4 \\
\hline $1001+203$ & Ton 1150 & No & 0.07498 & 1.0199 & $\ldots$ & $\ldots$ & $13.75 \pm 0.02$ & $13.78 \pm 0.03$ & 1 \\
\hline $1015-173$ & EC $10150-1722$ & Yes & 0.07258 & 1.0530 & $\ldots$ & $\ldots$ & $17.30 \pm 0.06$ & $17.25 \pm 0.07$ & 2,5 \\
\hline & & & 0.07510 & 1.0049 & $0.060 \pm 0.002$ & $131.26 \pm 0.67$ & $16.69 \pm 0.04$ & $16.74 \pm 0.05$ & 5 \\
\hline $1026+002$ & PG & No & 0.07485 & 1.0520 & $\ldots$ & $\ldots$ & $12.99 \pm 0.02$ & $13.00 \pm 0.03$ & 1 \\
\hline $1033+464$ & GD 123 & Yes & 0.07325 & 1.0083 & $\ldots$ & $\ldots$ & $14.72 \pm 0.02$ & $14.67 \pm 0.03$ & 2 \\
\hline & & & 0.07542 & 1.0048 & $0.731 \pm 0.001$ & $311.77 \pm 0.04$ & $14.34 \pm 0.02$ & $14.47 \pm 0.03$ & \\
\hline $1036-204$ & LHS 2293 & No & 0.07547 & 1.0150 & $\ldots$ & $\ldots$ & $15.32 \pm 0.02$ & $15.31 \pm 0.03$ & 1,7 \\
\hline $1037+512$ & PG & No & 0.07693 & 1.0375 & $\ldots$ & $\ldots$ & $15.03 \pm 0.02$ & $15.06 \pm 0.03$ & 1 \\
\hline $1049+103$ & PG & Yes & 0.07402 & 1.0349 & $\ldots$ & $\ldots$ & $16.05 \pm 0.02$ & $16.01 \pm 0.03$ & 2,5 \\
\hline & & & 0.07570 & 1.0311 & $0.262 \pm 0.001$ & $130.41 \pm 0.04$ & $14.87 \pm 0.02$ & $14.97 \pm 0.03$ & 5 \\
\hline $1051+516$ & SBS & No & 0.07483 & 1.0086 & $\ldots$ & $\ldots$ & $14.63 \pm 0.02$ & $14.68 \pm 0.03$ & 1 \\
\hline
\end{tabular}


Table 1

(Continued)

\begin{tabular}{|c|c|c|c|c|c|c|c|c|c|}
\hline WD & Name & $\begin{array}{c}\text { Spatially } \\
\text { Resolved? }\end{array}$ & $\begin{array}{l}\text { FWHM } \\
(\operatorname{arcsec})\end{array}$ & $a / b$ & $\begin{array}{c}\alpha \\
(\operatorname{arcsec})\end{array}$ & $\begin{array}{l}\text { P.A. } \\
\text { (deg) }\end{array}$ & $\begin{array}{c}\begin{array}{c}\text { F814W } \\
(\mathrm{mag})\end{array} \\
\end{array}$ & $\begin{array}{c}I_{c} \\
(\mathrm{mag})\end{array}$ & $\begin{array}{c}\text { Source } \\
\text { Notes }\end{array}$ \\
\hline \multirow[t]{2}{*}{$1106+316$} & Ton 28 & Yes & 0.07423 & 1.0118 & 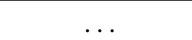 & 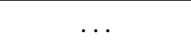 & $17.57 \pm 0.03$ & $17.52 \pm 0.04$ & 2 \\
\hline & & & 0.07560 & 1.0069 & $0.478 \pm 0.002$ & $104.94 \pm 0.20$ & $16.44 \pm 0.02$ & $16.49 \pm 0.03$ & \\
\hline $1106-211$ & & No & 0.07395 & 1.0109 & $\ldots$ & $\ldots$ & $15.60 \pm 0.02$ & $15.60 \pm 0.03$ & 1,7 \\
\hline \multirow{2}{*}{$1108+325^{b}$} & Ton 60 & Yes & 0.07355 & 1.0403 & $\ldots$ & $\ldots$ & $17.23 \pm 0.03$ & $17.17 \pm 0.04$ & 2,5 \\
\hline & & & 0.07325 & 1.0278 & $0.167 \pm 0.001$ & $298.36 \pm 0.12$ & $17.29 \pm 0.03$ & $17.34 \pm 0.04$ & 5 \\
\hline \multirow[t]{2}{*}{$1133+489$} & PG & Yes & 0.07483 & 1.0446 & $\ldots$ & $\ldots$ & $17.51 \pm 0.03$ & $17.45 \pm 0.04$ & 2,5 \\
\hline & & & 0.07738 & 1.0243 & $0.090 \pm 0.002$ & $10.31 \pm 0.32$ & $18.16 \pm 0.04$ & $18.29 \pm 0.05$ & 5 \\
\hline $1133+358$ & G147-65 & No & 0.07545 & 1.0109 & $\ldots$ & $\ldots$ & $13.21 \pm 0.02$ & $13.29 \pm 0.03$ & 1 \\
\hline $1140+004$ & & No & 0.07448 & 1.0070 & $\ldots$ & $\ldots$ & $16.93 \pm 0.02$ & $16.96 \pm 0.03$ & 1 \\
\hline $1156+132$ & LP 494-12 & $\ldots$ & $\ldots$ & $\ldots$ & $\ldots$ & $\ldots$ & $\ldots$ & $\ldots$ & \\
\hline \multirow[t]{2}{*}{$1156+129$} & & Yes & 0.07385 & 1.0305 & $\ldots$ & $\ldots$ & $18.02 \pm 0.04$ & $18.00 \pm 0.05$ & 2 \\
\hline & & & 0.07615 & 1.0346 & $0.564 \pm 0.002$ & $274.78 \pm 0.18$ & $16.20 \pm 0.02$ & $16.28 \pm 0.03$ & \\
\hline \multirow[t]{2}{*}{$1210+464$} & PG & Yes & 0.07270 & 1.0485 & $\ldots$ & $\ldots$ & $16.36 \pm 0.02$ & $16.31 \pm 0.03$ & 2 \\
\hline & & & 0.07343 & 1.0433 & $1.043 \pm 0.001$ & $324.32 \pm 0.05$ & $13.12 \pm 0.02$ & $13.16 \pm 0.03$ & \\
\hline \multirow[t]{2}{*}{$1218+497$} & PG & Yes & 0.07388 & 1.0301 & $\ldots$ & $\ldots$ & $16.73 \pm 0.02$ & $16.68 \pm 0.03$ & 2,5 \\
\hline & & & 0.07595 & 1.0247 & $0.303 \pm 0.001$ & $335.14 \pm 0.06$ & $16.14 \pm 0.02$ & $16.22 \pm 0.03$ & 5 \\
\hline \multirow[t]{2}{*}{$1236-004$} & & Yes & 0.07400 & 1.0155 & $\ldots$ & $\ldots$ & $18.10 \pm 0.04$ & $18.05 \pm 0.05$ & 2 \\
\hline & & & 0.07440 & 1.0312 & $0.663 \pm 0.002$ & $267.12 \pm 0.20$ & $17.83 \pm 0.04$ & $17.89 \pm 0.05$ & \\
\hline $1247+550$ & LHS 342 & No & 0.07520 & 1.0132 & $\ldots$ & $\ldots$ & $16.37 \pm 0.02$ & $16.37 \pm 0.03$ & 1,7 \\
\hline $1247-176$ & EC $12477-1738$ & No & 0.07468 & 1.0336 & $\ldots$ & $\ldots$ & $14.82 \pm 0.02$ & $14.86 \pm 0.03$ & 1 \\
\hline \multirow[t]{2}{*}{$1307-141$} & EC $13077-1411$ & Yes & 0.07350 & 1.0111 & $\ldots$ & $\ldots$ & $16.90 \pm 0.02$ & $16.86 \pm 0.03$ & 2 \\
\hline & & & 0.07670 & 1.0218 & $2.133 \pm 0.001$ & $165.51 \pm 0.03$ & $15.32 \pm 0.02$ & $15.40 \pm 0.03$ & \\
\hline \multirow[t]{2}{*}{$1333+487$} & GD 325 & Yes & 0.07542 & 1.0197 & $\ldots$ & $\ldots$ & $14.19 \pm 0.02$ & $14.17 \pm 0.03$ & 2 \\
\hline & & & 0.07500 & 1.0174 & $2.950 \pm 0.001$ & $72.08 \pm 0.01$ & $13.35 \pm 0.02$ & $13.43 \pm 0.03$ & \\
\hline $1333+005$ & LP 618-14 & No & 0.07728 & 1.0302 & $\ldots$ & $\ldots$ & $15.59 \pm 0.02$ & $15.62 \pm 0.03$ & 1 \\
\hline $1334-326$ & EC $13349-3237$ & No & 0.07345 & 1.0648 & $\ldots$ & $\ldots$ & $15.03 \pm 0.02$ & $15.04 \pm 0.03$ & 1 \\
\hline $1339+606$ & SBS & No & 0.07388 & 1.0424 & $\ldots$ & $\ldots$ & $16.49 \pm 0.02$ & $16.49 \pm 0.03$ & 1 \\
\hline \multirow[t]{2}{*}{$1339+346$} & PG & Yes & 0.07335 & 1.0288 & $\ldots$ & $\ldots$ & $16.06 \pm 0.02$ & $16.02 \pm 0.03$ & 2,7 \\
\hline & & & 0.07527 & 1.0320 & $2.521 \pm 0.001$ & $212.69 \pm 0.02$ & $14.71 \pm 0.02$ & $14.70 \pm 0.03$ & 8 \\
\hline \multirow{2}{*}{$1412-049$} & PG & Yes & 0.07208 & 1.0433 & $\ldots$ & $\ldots$ & $17.12 \pm 0.03$ & $17.06 \pm 0.04$ & 2 \\
\hline & & & 0.07513 & 1.0340 & $3.507 \pm 0.001$ & $255.50 \pm 0.02$ & $14.79 \pm 0.02$ & $14.81 \pm 0.03$ & \\
\hline \multirow{2}{*}{$1419+576$} & SBS & Yes & 0.07475 & 1.0167 & $\ldots$ & $\ldots$ & $17.52 \pm 0.03$ & $17.48 \pm 0.04$ & 2 \\
\hline & & & 0.07672 & 1.1125 & $0.655 \pm 0.002$ & $304.04 \pm 0.12$ & $15.06 \pm 0.02$ & $15.10 \pm 0.03$ & 4 \\
\hline $1433+538$ & GD 337 & No & 0.07440 & 1.0056 & $\ldots$ & $\ldots$ & $15.67 \pm 0.02$ & $15.67 \pm 0.03$ & 1 \\
\hline $1434+289$ & Ton 210 & No & 0.07425 & 1.0381 & $\ldots$ & $\ldots$ & $16.08 \pm 0.02$ & $16.02 \pm 0.03$ & 1,7 \\
\hline $1435+370$ & CBS 194 & Yes & 0.07253 & 1.0629 & $\ldots$ & $\ldots$ & $16.94 \pm 0.02$ & $16.90 \pm 0.03$ & 2 \\
\hline & & & 0.07513 & 1.0268 & $1.253 \pm 0.001$ & $318.95 \pm 0.05$ & $14.81 \pm 0.02$ & $14.86 \pm 0.03$ & \\
\hline $1436-216$ & EC $14363-2137$ & No & 0.07530 & 1.0267 & $\ldots$ & $\ldots$ & $14.48 \pm 0.02$ & $14.51 \pm 0.03$ & 1 \\
\hline $1443+336$ & PG & Yes & 0.07190 & 1.0299 & $\ldots$ & $\ldots$ & $16.91 \pm 0.02$ & $16.86 \pm 0.03$ & 2 \\
\hline & & & 0.07603 & 1.0177 & $0.679 \pm 0.001$ & $286.25 \pm 0.09$ & $15.53 \pm 0.02$ & $15.57 \pm 0.03$ & \\
\hline $1458+171$ & PG & No & 0.07473 & 1.0231 & $\ldots$ & $\ldots$ & $15.79 \pm 0.02$ & $15.79 \pm 0.03$ & 1 \\
\hline $1502+349$ & CBS 223 & Yes & 0.07478 & 1.0238 & $\cdots$ & $\cdots$ & $16.85 \pm 0.02$ & $16.81 \pm 0.03$ & 2 \\
\hline & & & 0.07628 & 1.0310 & $1.912 \pm 0.002$ & $30.13 \pm 0.04$ & $17.04 \pm 0.03$ & $17.16 \pm 0.04$ & \\
\hline $1504+546$ & CBS 301 & No & 0.07555 & 1.0352 & $\ldots$ & $\ldots$ & $15.01 \pm 0.02$ & $15.04 \pm 0.03$ & 1 \\
\hline $1517+502$ & CBS 311 & No & 0.07483 & 1.0398 & $\ldots$ & $\ldots$ & $16.66 \pm 0.02$ & $16.68 \pm 0.03$ & 1 \\
\hline $1522+508$ & CBS 318 & No & 0.07560 & 1.0307 & $\ldots$ & $\ldots$ & $15.96 \pm 0.02$ & $16.00 \pm 0.03$ & 1 \\
\hline $1558+616$ & HS $1558+6140$ & Yes & 0.07465 & 1.0234 & $\ldots$ & $\ldots$ & $17.27 \pm 0.03$ & $17.23 \pm 0.04$ & 2 \\
\hline & & & 0.07480 & 1.0224 & $0.719 \pm 0.001$ & $336.00 \pm 0.10$ & $15.71 \pm 0.02$ & $15.80 \pm 0.03$ & \\
\hline $1603+125$ & & No & 0.07248 & 1.0349 & $\ldots$ & $\ldots$ & $14.18 \pm 0.02$ & $14.17 \pm 0.03$ & 1,7 \\
\hline $1619+525$ & PG & Yes & 0.07340 & 1.0187 & $\ldots$ & $\ldots$ & $15.81 \pm 0.02$ & $15.77 \pm 0.03$ & 3 \\
\hline & & & 0.07445 & 1.0112 & $2.593 \pm 0.001$ & $282.58 \pm 0.02$ & $15.20 \pm 0.02$ & $15.24 \pm 0.03$ & \\
\hline & & & 0.07545 & 1.0378 & $0.465 \pm 0.002$ & $24.02 \pm 0.22$ & $17.84 \pm 0.04$ & $17.96 \pm 0.05$ & \\
\hline $1619+414$ & KUV 16195+4125 & Yes & 0.07443 & 1.0105 & $\ldots$ & $\ldots$ & $17.38 \pm 0.03$ & $17.35 \pm 0.04$ & 2,5 \\
\hline & & & 0.07630 & 1.0074 & $0.232 \pm 0.001$ & $188.48 \pm 0.09$ & $15.68 \pm 0.02$ & $15.80 \pm 0.03$ & 5 \\
\hline $1622+323$ & PG & Yes & 0.07478 & 1.0414 & $\ldots$ & $\ldots$ & $16.92 \pm 0.03$ & $16.86 \pm 0.04$ & 2,5 \\
\hline & & & 0.07420 & 1.0408 & $0.093 \pm 0.002$ & $298.18 \pm 0.37$ & $15.77 \pm 0.02$ & $15.81 \pm 0.03$ & 5 \\
\hline $1631+781$ & RX J1629.0+7804 & Yes & 0.07435 & 1.0082 & $\ldots$ & $\ldots$ & $13.61 \pm 0.02$ & $13.56 \pm 0.03$ & 2,5 \\
\hline & & & 0.07570 & 1.1008 & $0.302 \pm 0.001$ & $175.96 \pm 0.10$ & $12.28 \pm 0.02$ & $12.33 \pm 0.03$ & 4,5 \\
\hline $1643+143$ & PG & Yes & 0.07428 & 1.0093 & $\ldots$ & $\ldots$ & $16.27 \pm 0.02$ & $16.22 \pm 0.03$ & 2,5 \\
\hline & & & 0.07510 & 1.0129 & $0.312 \pm 0.001$ & $20.87 \pm 0.06$ & $13.91 \pm 0.02$ & $13.95 \pm 0.03$ & 5 \\
\hline $1646+062$ & PG & Yes & 0.07308 & 1.0284 & $\ldots$ & $\ldots$ & $16.45 \pm 0.03$ & $16.40 \pm 0.04$ & 2,5 \\
\hline & & & 0.07433 & 1.0327 & $0.161 \pm 0.001$ & $269.03 \pm 0.14$ & $15.42 \pm 0.02$ & $15.49 \pm 0.03$ & 5 \\
\hline $1658+440$ & PG & No & 0.07345 & 1.0213 & $\ldots$ & $\ldots$ & $15.09 \pm 0.02$ & $15.04 \pm 0.03$ & 1 \\
\hline $1717-345$ & & No & 0.07617 & 1.0086 & $\ldots$ & $\ldots$ & $14.17 \pm 0.02$ & $14.22 \pm 0.03$ & 1 \\
\hline
\end{tabular}


Table 1

(Continued)

\begin{tabular}{|c|c|c|c|c|c|c|c|c|c|}
\hline WD & Name & $\begin{array}{c}\text { Spatially } \\
\text { Resolved? }\end{array}$ & $\begin{array}{l}\text { FWHM } \\
(\operatorname{arcsec})\end{array}$ & $a / b$ & $\begin{array}{c}\alpha \\
(\operatorname{arcsec})\end{array}$ & $\begin{array}{l}\text { P.A. } \\
(\mathrm{deg})\end{array}$ & $\begin{array}{c}\mathrm{F}^{8} 14 \mathrm{~W}^{\mathrm{a}} \\
(\mathrm{mag})\end{array}$ & $\begin{array}{c}I_{c} \\
(\mathrm{mag})\end{array}$ & $\begin{array}{c}\text { Source } \\
\text { Notes } \\
\end{array}$ \\
\hline \multirow[t]{3}{*}{$1833+644$} & KUV 18332+6429 & Yes & 0.07360 & 1.0672 & & & $17.31 \pm 0.04$ & $17.26 \pm 0.05$ & 3 \\
\hline & & & 0.07588 & 1.0400 & $0.079 \pm 0.002$ & $221.91 \pm 0.48$ & $15.37 \pm 0.02$ & $15.43 \pm 0.03$ & \\
\hline & & & 0.07700 & 1.0489 & $1.820 \pm 0.002$ & $129.47 \pm 0.03$ & $19.60 \pm 0.08$ & $19.83 \pm 0.09$ & \\
\hline $1845+683$ & KUV 18453+6819 & No & 0.07343 & 1.0098 & $\ldots$ & $\ldots$ & $15.67 \pm 0.02$ & $15.61 \pm 0.03$ & 1,7 \\
\hline $2009+622$ & GD 543 & No & 0.07468 & 1.0291 & $\ldots$ & $\ldots$ & $15.03 \pm 0.02$ & $15.02 \pm 0.03$ & 1 \\
\hline \multirow[t]{2}{*}{$2151-015$} & LTT 8747 & Yes & 0.07443 & 1.0252 & $\ldots$ & $\ldots$ & $14.32 \pm 0.02$ & $14.29 \pm 0.03$ & 2 \\
\hline & & & 0.07790 & 1.0227 & $1.082 \pm 0.001$ & $193.78 \pm 0.03$ & $15.06 \pm 0.02$ & $15.31 \pm 0.03$ & \\
\hline $2211+372$ & LHS 3779 & No & 0.07228 & 1.0119 & $\ldots$ & $\ldots$ & $16.40 \pm 0.02$ & $16.38 \pm 0.03$ & 1,7 \\
\hline \multirow[t]{2}{*}{$2237-365$} & HE $2237-3630$ & $\ldots$ & $\ldots$ & $\ldots$ & $\ldots$ & $\ldots$ & $\ldots$ & $\ldots$ & \\
\hline & LHS 3841 & No & 0.07558 & 1.0107 & $\ldots$ & $\ldots$ & $14.86 \pm 0.02$ & $14.90 \pm 0.03$ & 1 \\
\hline $2257+162$ & PG & No & 0.07500 & 1.0081 & $\ldots$ & $\ldots$ & $16.06 \pm 0.02$ & $16.04 \pm 0.03$ & 1 \\
\hline $2317+268$ & KUV $23176+2650$ & No & 0.07492 & 1.0257 & $\ldots$ & $\ldots$ & $15.64 \pm 0.02$ & $15.65 \pm 0.03$ & 1 \\
\hline $2323+256$ & G128-62 & No & 0.07428 & 1.0224 & $\ldots$ & $\ldots$ & $16.30 \pm 0.02$ & $16.28 \pm 0.03$ & 1,7 \\
\hline $2336-187$ & G273-97 & No & 0.07483 & 1.0049 & $\ldots$ & $\ldots$ & $15.18 \pm 0.02$ & $15.16 \pm 0.03$ & 1,7 \\
\hline $2349-283$ & GD 1617 & No & 0.07358 & 1.0107 & $\ldots$ & $\ldots$ & $15.65 \pm 0.02$ & $15.61 \pm 0.03$ & 1,7 \\
\hline
\end{tabular}

Notes. (1) Single point source; (2) double point source, two Airy disks; (3) triple point source, three Airy disks; (4) single but elongated Airy disk; (5) measurements affected by $\alpha<0$ '.4; (6) common proper motion companion; (7) not a white dwarf binary (the Appendix, Table 8); (8) background star, optical but not physical companion.

a All photometry is in Vega magnitudes.

${ }^{\mathrm{b}}$ The component identification in this system was not unambiguous. See the Appendix for details.

measured directly. Tables 4 and 5 list the parameters derived for all low-mass companions. Spectral type estimates were assigned based on the $I-K$ color, with empirical absolute magnitude, color, and spectral type relations for $\mathrm{M}$ dwarfs taken from Dahn et al. (2002), Kirkpatrick \& McCarthy (1994), and Leggett (1992). The photometric distances for these stars were calculated from the average of $I-M_{I}$ and $K-M_{K}$.

\section{RESULTS}

Of the 72 certain or highly probable white dwarf binary systems, the ACS images spatially resolve 43 systems into two or more components, a fraction of $60 \%$. Given that the spatial resolution of 2MASS is a few arcseconds, and the targets were all selected for near-infrared excess in their 2MASS photometry, the wide binary fraction among white dwarfs with low-mass companions is at least $60 \%$. These results can be compared with the extensive ground-based study of Farihi (2004), where it was found that white dwarf-M dwarf binaries with angular separations smaller than 4 " represent $63 \%$ of all such systems. Because the ACS program targeted stars in this latter group, one can estimate (all else assumed equal) that $75 \%$ $(0.37+(0.60 \times 0.63))$ of all low-mass companions to white dwarfs are widely separated and never experienced a common envelope phase. It then follows that around $25 \%$ of all such binaries are post-common envelope systems, consistent with the findings of Rebassa-Mansergas et al. (2007) based on a similar number of SDSS spectra.

\subsection{Three Very Close Visual Doubles}

Figures 2 and 3 demonstrate there are a few sources whose FWHM or $a / b$ values are clear outliers. The three objects with $a / b>1.1$ in Figure 3 are particularly interesting and are just discernibly (by human eye) elongated with respect to their visual companions. Each of these three sources is a visual $\mathrm{M}$ dwarf companion to a white dwarf, whose PSF is point-like and narrow in comparison. With little difficulty, daophot was able to fit two sources to each of these elongated PSFs, confirming they are all barely resolved binaries at angular separations around 0'03 (the

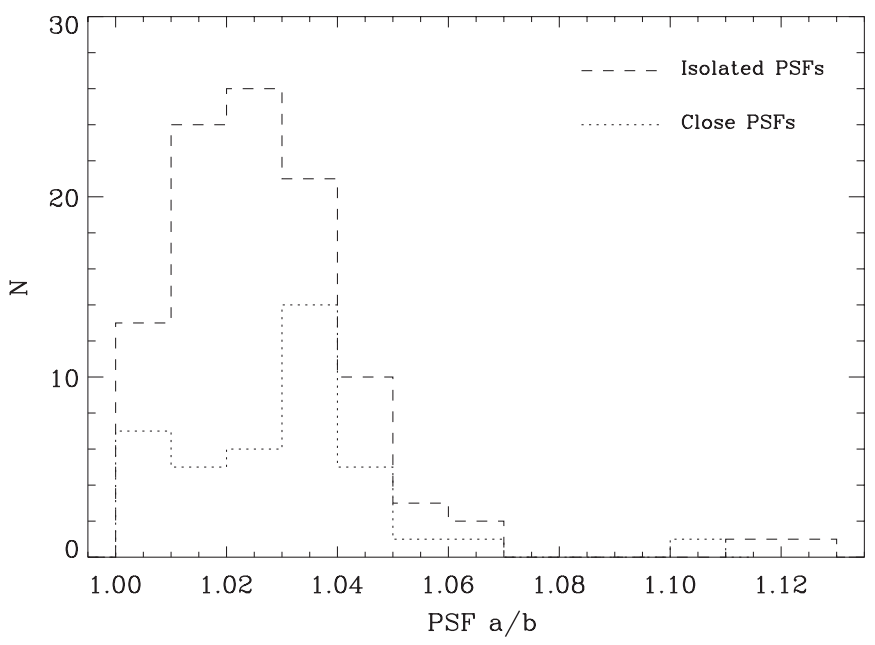

Figure 3. Histogram of the PSF semimajor to semiminor axis. The dashed line is for stellar images that are well isolated from any companions, while the dotted line is for sources with companions within 0."4.

separations of these three close doubles were underestimated in Paper I). Photometry and astrometry for these three binary $\mathrm{M}$ dwarfs is listed in Table 6; these measurements demonstrate that binaries with $\Delta I \lesssim 1 \mathrm{mag}$ and with separations as small as 1 pixel were readily detectable in this survey.

\subsection{Photometric Distances: A Need for Better Agreement}

In order to convert angular separation in arcseconds into a projected orbital separation in $\mathrm{AU}$, a distance estimate is necessary; only four stars in this survey have reliable trigonometric parallaxes (McCook \& Sion 2008). Unfortunately, the white dwarf and $\mathrm{M}$ dwarf components within each binary often give discrepant distance estimates when obtained using other methods. To measure this effect, define the quantity:

$$
\Delta(m-M)=(m-M)_{\mathrm{wd}}-(m-M)_{\mathrm{rd}} .
$$

There is a significant mismatch in photometric distances when $|\Delta(m-M)|>0.5 \mathrm{mag}$, corresponding to over 
Table 2

Primary Target Coordinates

\begin{tabular}{|c|c|c|c|}
\hline WD & $\begin{array}{l}\text { R.A. } \\
(\mathrm{h} \mathrm{m} \mathrm{s})\end{array}$ & $\begin{array}{l}\text { Decl. } \\
\left({ }^{\prime \prime \prime}\right)\end{array}$ & $\begin{array}{c}\text { Epoch } \\
(\mathrm{yr})\end{array}$ \\
\hline $0023+388$ & 002633.2 & +390903 & 2004.56 \\
\hline $0034-211$ & 003724.9 & -205343 & 2004.56 \\
\hline $0116-231$ & 011837.2 & -225459 & 2004.67 \\
\hline $0131-163$ & 013424.1 & -160708 & 2004.67 \\
\hline $0145-221$ & 014721.8 & -215651 & 2005.43 \\
\hline $0145+257$ & 014808.1 & -253244 & 2004.71 \\
\hline $0205+133$ & 020803.5 & +133625 & 2004.66 \\
\hline $0208-153$ & 021043.0 & -150633 & 2004.55 \\
\hline $0219+282$ & 022228.4 & +283008 & 2004.62 \\
\hline $0237+115$ & 024006.7 & +114830 & 2004.60 \\
\hline $0257-005$ & 030024.5 & -002342 & 2005.50 \\
\hline $0303-007$ & 030607.1 & -003114 & 2004.56 \\
\hline $0309-275$ & 031133.1 & -271925 & 2005.43 \\
\hline $0324+738$ & 033013.9 & +740157 & 2004.70 \\
\hline $0331-356$ & 033352.4 & -353118 & 2005.37 \\
\hline $0347-137$ & 035014.6 & -133514 & 2004.57 \\
\hline $0354+463$ & 035817.1 & +462841 & 2004.70 \\
\hline $0357-233$ & 035904.9 & -231225 & 2004.67 \\
\hline $0430+136$ & 043310.3 & +134517 & 2005.16 \\
\hline $0458-665$ & 045854.0 & -662813 & 2004.62 \\
\hline $0518+333$ & 052143.5 & +332159 & 2005.16 \\
\hline $0752-146$ & 075508.9 & -144553 & 2005.22 \\
\hline $0807+190^{\mathrm{a}}$ & 081000.3 & +185147 & 2005.33 \\
\hline $0812+478$ & 081548.9 & +474039 & 2005.18 \\
\hline $0824+288$ & 082704.9 & +284403 & 2005.17 \\
\hline $0908+226$ & 091143.1 & +222749 & 2005.17 \\
\hline $0915+201$ & 091833.0 & +195308 & 2005.18 \\
\hline $0933+025$ & 093540.7 & +022158 & 2005.17 \\
\hline $0937-095^{\mathrm{a}}$ & 093949.8 & -094611 & 2005.20 \\
\hline $0949+451$ & 095221.9 & +445431 & 2004.72 \\
\hline $1001+203$ & 100404.2 & +200923 & 2005.29 \\
\hline $1015-173$ & 101728.8 & -173707 & 2005.23 \\
\hline $1026+002$ & 102834.9 & -000030 & 2005.29 \\
\hline $1033+464$ & 103625.2 & +460831 & 2005.20 \\
\hline $1036-204$ & 103855.3 & -204055 & 2005.25 \\
\hline $1037+512$ & 104016.8 & +515648 & 2005.19 \\
\hline $1049+103$ & 105227.7 & +100338 & 2005.23 \\
\hline $1051+516$ & 105421.9 & +512254 & 2004.73 \\
\hline $1106+316$ & 110843.0 & +312356 & 2005.17 \\
\hline $1106-211^{\mathrm{a}}$ & 110910.9 & -212333 & 2005.24 \\
\hline $1108+325$ & 111045.9 & +321447 & 2005.32 \\
\hline $1133+489$ & 113609.5 & +484319 & 2004.56 \\
\hline $1133+358$ & 113542.7 & +353424 & 2005.22 \\
\hline $1140+004$ & 114312.5 & +000926 & 2005.33 \\
\hline \multirow[t]{2}{*}{$1156+132^{b}$} & 115932.6 & +130013 & 2005.19 \\
\hline & 115933.1 & +130032 & \\
\hline $1156+129$ & 115915.6 & +123929 & 2005.41 \\
\hline $1210+464$ & 121259.8 & +460947 & 2005.18 \\
\hline $1218+497$ & 122105.3 & +492720 & 2004.56 \\
\hline $1236-004$ & 123836.4 & -004043 & 2004.55 \\
\hline $1247+550$ & 125007.5 & +544700 & 2004.66 \\
\hline $1247-176$ & 125022.1 & -175448 & 2005.18 \\
\hline $1307-141$ & 131022.5 & -142708 & 2005.26 \\
\hline $1333+487$ & 133601.7 & +482845 & 2004.64 \\
\hline $1333+005$ & 133616.0 & +001731 & 2004.56 \\
\hline $1334-326$ & 133750.6 & -325222 & 2005.17 \\
\hline $1339+606$ & 134100.0 & +602610 & 2004.58 \\
\hline $1339+346$ & 134118.1 & +342155 & 2005.24 \\
\hline $1412-049$ & 141502.5 & -051103 & 2004.56 \\
\hline $1419+576$ & 142105.2 & +572456 & 2004.70 \\
\hline $1433+538$ & 143443.1 & +533525 & 2004.56 \\
\hline $1434+289$ & 143641.6 & +284452 & 2005.25 \\
\hline $1435+370$ & 143736.7 & +365135 & 2004.59 \\
\hline $1436-216$ & 143912.8 & -215012 & 2005.18 \\
\hline $1443+336$ & 144600.6 & +332850 & 2004.67 \\
\hline
\end{tabular}

Table 2

(Continued)

\begin{tabular}{|c|c|c|c|}
\hline WD & $\begin{array}{l}\text { R.A. } \\
(\mathrm{h} \mathrm{m} \mathrm{s})\end{array}$ & $\begin{array}{l}\text { Decl. } \\
\left({ }^{\prime \prime \prime \prime}\right)\end{array}$ & $\begin{array}{c}\text { Epoch } \\
(\mathrm{yr})\end{array}$ \\
\hline $1458+171$ & 150019.4 & +165916 & 2004.57 \\
\hline $1502+349$ & 150431.8 & +344701 & 2004.70 \\
\hline $1504+546$ & 150605.3 & +542819 & 2004.56 \\
\hline $1517+502$ & 151905.9 & +500703 & 2004.56 \\
\hline $1522+508$ & 152425.2 & +504011 & 2005.22 \\
\hline $1558+616$ & 155855.4 & +613203 & 2004.66 \\
\hline $1603+125^{\mathrm{a}}$ & 160532.1 & +122542 & 2004.61 \\
\hline $1619+525$ & 162024.5 & +522322 & 2004.62 \\
\hline $1619+414$ & 162112.6 & +411810 & 2004.64 \\
\hline $1622+323$ & 162449.0 & +321702 & 2004.62 \\
\hline $1631+781$ & 162909.9 & +780440 & 2004.64 \\
\hline $1643+143$ & 164539.2 & +141745 & 2005.23 \\
\hline $1646+062$ & 164907.8 & +060846 & 2004.64 \\
\hline $1658+440$ & 165948.4 & +440105 & 2005.26 \\
\hline $1717-345$ & 172110.3 & -343328 & 2005.20 \\
\hline $1833+644$ & 183329.2 & +643152 & 2005.19 \\
\hline $1845+683$ & 184509.2 & +682234 & 2004.66 \\
\hline $2009+622$ & 201042.6 & +622531 & 2004.73 \\
\hline $2151-015$ & 215406.5 & -011711 & 2004.59 \\
\hline $2211+372$ & 221408.9 & +37 2711 & 2004.63 \\
\hline \multirow[t]{2}{*}{$2237-365^{b}$} & 223959.4 & -361601 & 2004.64 \\
\hline & 224005.3 & -361519 & $\ldots$ \\
\hline $2257+162$ & 225946.9 & +162917 & 2005.45 \\
\hline $2317+268$ & 232004.1 & +260623 & 2004.58 \\
\hline $2323+256$ & 232557.9 & +255221 & 2004.66 \\
\hline $2336-187$ & 233852.8 & -182614 & 2005.32 \\
\hline $2349-283$ & 235223.2 & -280316 & 2004.65 \\
\hline
\end{tabular}

Notes.

a Not a white dwarf (Appendix, Table 8).

b These white dwarfs were not imaged by ACS; the first row of coordinates gives the position of the imaged (incorrect) star, while the second set of coordinates gives the correct position for the white dwarf.

$25 \%$ disagreement between distance estimates. In this view, exactly half of the binaries without parallaxes have significant discrepancies in the estimates obtained from each component.

Figure 4 plots a histogram of this distance modulus discrepancy, demonstrating that the most common type of mismatch is for $\Delta(m-M)>+0.5$ mag; i.e., the white dwarf appears to be further from the Sun and overluminous relative to the $\mathrm{M}$ dwarf. In comparing parameters derived from simultaneous spectral fitting of both white dwarf and $\mathrm{M}$ dwarf components in a composite spectrum versus fitting only the white dwarf (as generally done in the literature for the systems in this study), Tappert et al. (2009) find this same bias. They conclude that when Balmer lines are uncorrected for the $\mathrm{M}$ dwarf contribution, the resulting equivalent widths are underestimated, leading to an overestimated temperature and luminosity. There is little doubt this effect is present here, and it underscores the need for reliable and robust white dwarf parameter determinations for these systems.

Another source of error in the photometric distances lies in the fact that up to one dozen white dwarfs in this study have little or no observational constraints on their effective temperatures or surface gravities (McCook \& Sion 2008). While there is intrinsic scatter in the absolute magnitudes of $\mathrm{M}$ dwarfs (Kirkpatrick \& McCarthy 1994; Leggett 1992) that depends primarily on metallicity (difficult to measure for cool stars), it is likely that many of the distances discrepancies would be improved with a standard optical spectral analysis of the white dwarfs. 
Table 3

White Dwarf Parameters

\begin{tabular}{|c|c|c|c|c|c|}
\hline WD & $\begin{array}{l}T_{\text {eff }} \\
(\mathrm{K})\end{array}$ & $\begin{array}{c}\log g \\
\left(\mathrm{~cm} \mathrm{~s}^{-2}\right)\end{array}$ & $\begin{array}{c}V \\
(\mathrm{mag})\end{array}$ & $\begin{array}{l}d_{\mathrm{wd}} \\
(\mathrm{pc})\end{array}$ & References \\
\hline $0023+388$ & 10800 & 8.14 & 15.97 & 59 & 1,2 \\
\hline $0034-211$ & 17200 & 8.04 & 14.89 & 61 & 1,2 \\
\hline $0116-231$ & 25000 & 7.9 & 16.29 & 164 & 3 \\
\hline $0131-163$ & 49000 & 7.81 & 13.94 & 105 & 2 \\
\hline $0145-221$ & 11600 & 8.10 & 14.85 & 39 & 4,5 \\
\hline $0145-257$ & 26200 & 7.93 & 14.66 & 80 & 6,7 \\
\hline $0205+133$ & 57400 & 7.63 & 15.06 & 223 & 8 \\
\hline $0208-153$ & 20000 & 7.9 & 15.69 & 101 & 1 \\
\hline \multirow[t]{2}{*}{$0219+282$} & 36300 & 8.09 & 16.97 & 310 & 1,9 \\
\hline & 27200 & 8.09 & $\ldots$ & $\ldots$ & 9 \\
\hline $0237+115$ & 70000 & 8.0 & 15.97 & 273 & 1,10 \\
\hline $0257-005$ & 80900 & 7.13 & 16.97 & 1040 & 1,11 \\
\hline $0303-007$ & 18700 & 7.97 & 16.59 & 144 & 2 \\
\hline $0309-275$ & 40000 & 7.8 & 16.46 & 292 & 1 \\
\hline $0324+738$ & 8500 & 8.65 & 16.85 & 40 & 1 \\
\hline $0331-356$ & 31400 & 7.70 & 15.11 & 141 & 4 \\
\hline $0347-137$ & 15500 & 8.0 & 15.32 & 66 & 12 \\
\hline $0354+463$ & 8000 & 8.0 & 15.55 & 32 & 1,12 \\
\hline $0357-233$ & 35000 & 7.9 & 15.87 & 199 & 1,13 \\
\hline $0430+136$ & 36000 & 7.90 & 17.33 & 376 & 1,2 \\
\hline $0458-665$ & 20000 & 7.9 & 17.72 & 258 & 14 \\
\hline $0518+333$ & 9500 & 7.77 & 16.10 & 65 & 1,11 \\
\hline $0752-146$ & 18500 & 8.0 & 13.53 & 35 & 1,15 \\
\hline $0812+478$ & 60900 & 7.58 & 15.22 & 259 & 8 \\
\hline $0824+288$ & 50700 & 7.74 & 14.80 & 168 & 1,8 \\
\hline $0908+226$ & 15000 & 8.0 & 19.22 & 402 & 1 \\
\hline $0915+201$ & 70000 & 7.33 & 16.52 & 643 & 8 \\
\hline $0933+025$ & 22400 & 8.04 & 16.24 & 135 & 8 \\
\hline $0949+451$ & 11000 & 8.0 & 15.89 & 65 & 1 \\
\hline $1001+203$ & 21500 & 7.97 & 15.91 & 117 & 8 \\
\hline $1015-173$ & 25000 & 7.9 & 16.99 & 226 & 1 \\
\hline $1026+002$ & 17200 & 7.97 & 13.95 & 40 & 8 \\
\hline $1033+464$ & 29400 & 7.88 & 14.37 & 83 & 8 \\
\hline $1036-204$ & 7500 & 8.0 & 16.28 & 29 & 16,11 \\
\hline $1037+512$ & 20100 & 8.03 & 16.38 & 133 & 8 \\
\hline $1049+103$ & 20600 & 7.91 & 15.79 & 112 & 1,8 \\
\hline $1051+516$ & 20000 & 7.9 & 17.42 & 225 & 1,17 \\
\hline $1106+316$ & 25000 & 7.9 & 17.26 & 257 & 1,18 \\
\hline $1108+325$ & 63000 & 7.59 & 16.83 & 550 & 1,8 \\
\hline $1133+358$ & 6500 & 7.67 & 16.87 & 50 & $1,19,20$ \\
\hline $1133+489$ & 48000 & 8.00 & 17.12 & 384 & 1,21 \\
\hline $1140+004$ & 16000 & 8.0 & 18.30 & 278 & 1,17 \\
\hline $1156+129$ & 15000 & 8.0 & 17.85 & 214 & 1 \\
\hline $1210+464$ & 27700 & 7.85 & 16.03 & 171 & 8 \\
\hline $1218+497$ & 35700 & 7.87 & 16.36 & 250 & 1,8 \\
\hline $1236-004$ & 44700 & 7.60 & 17.72 & 673 & 17 \\
\hline $1247-176$ & 20900 & 8.06 & 16.50 & 141 & 22 \\
\hline $1307-141$ & 20000 & 7.9 & 16.65 & 158 & 1 \\
\hline $1333+487$ & 16000 & 8.20 & 14.15 & 35 & 23,24 \\
\hline $1333+005$ & 7500 & 8.0 & 17.47 & 69 & 1,25 \\
\hline $1334-326$ & 35000 & 7.52 & 17.29 & 619 & 4,26 \\
\hline $1339+606$ & 46300 & 7.80 & 17.10 & 439 & 17 \\
\hline $1339+346$ & 16000 & 7.82 & 15.93 & 103 & 8 \\
\hline $1412-049$ & 50000 & 7.8 & 16.73 & 398 & 1 \\
\hline $1419+576$ & 25000 & 7.9 & 17.27 & 258 & 1 \\
\hline $1433+358$ & 22400 & 7.80 & 16.13 & 151 & 8 \\
\hline $1434+289$ & 32800 & 8.00 & 15.71 & 156 & 8 \\
\hline $1435+370$ & 15300 & 7.99 & 16.75 & 129 & 2 \\
\hline $1436-216$ & 25000 & 7.9 & 16.64 & 193 & 1 \\
\hline $1443+336$ & 29800 & 7.83 & 16.56 & 239 & 8 \\
\hline $1458+171$ & 22000 & 7.43 & 16.39 & 226 & 8 \\
\hline $1502+349$ & 21300 & 7.96 & 16.58 & 161 & 2 \\
\hline $1504+546$ & 24700 & 7.86 & 17.07 & 243 & 2 \\
\hline $1517+502$ & 31100 & 7.84 & 17.95 & 406 & 27 \\
\hline $1522+508$ & 21600 & 8.10 & 17.65 & 238 & 17 \\
\hline
\end{tabular}

Table 3

(Continued)

\begin{tabular}{ccccrc}
\hline \hline WD & $\begin{array}{c}T_{\text {eff }} \\
(\mathrm{K})\end{array}$ & $\begin{array}{c}\log g \\
\left(\mathrm{~cm} \mathrm{~s}^{-2}\right)\end{array}$ & $\begin{array}{c}V \\
(\mathrm{mag})\end{array}$ & $\begin{array}{c}d_{\text {wd }} \\
(\mathrm{pc})\end{array}$ & References \\
\hline $1558+616$ & 15000 & 8.0 & 16.95 & 141 & 1 \\
$1619+525$ & 18000 & 7.90 & 15.62 & 94 & 1,8 \\
$1619+414$ & 14100 & 7.93 & 17.22 & 156 & 1,2 \\
$1622+323$ & 68300 & 7.56 & 16.52 & 513 & 1,8 \\
$1631+781$ & 44900 & 7.76 & 13.23 & 75 & 1,2 \\
$1643+143$ & 26800 & 7.91 & 15.95 & 153 & 1,8 \\
$1646+062$ & 29900 & 7.98 & 16.10 & 174 & 1,8 \\
$1717-345$ & 12700 & 8.0 & 17.20 & 137 & 1,28 \\
$1833+644$ & 25000 & 7.9 & 16.99 & 227 & 1 \\
$2009+622$ & 25900 & 7.70 & 15.29 & 125 & 1,29 \\
$2151-015$ & 9100 & 8.21 & 14.54 & 22 & 1,2 \\
$2257+162$ & 24600 & 7.49 & 16.14 & 213 & 8 \\
$2317+268$ & 31500 & 7.70 & 16.63 & 284 & $1,2,30$ \\
$2336-187$ & 8100 & 8.05 & 15.51 & 31 & 4 \\
\hline
\end{tabular}

Notes. A single digit following the decimal place for $\log g$ indicates an assumption of $M=0.60 M_{\odot}$. White dwarf $V$-band magnitudes are disentangled from the light of their companions, by virtue of being derived via: (1) spatially resolved photometry; (2) effective temperature, model colors, and uncontaminated photometry in other bandpasses (e.g., F814W, I); or (3) best estimates based on photographic photometry when no other data are available. Distances were calculated from absolute magnitudes using white dwarf models (Bergeron et al. 1995a, 1995b) and specified references.

References. (1) This work and Paper I; (2) Trembley \& Bergeron 2007; (3) Wolff et al. 1996; (4) Koester et al. 2001; (5) Fontaine et al. 2003; (6) Finley et al. 1997; (7) Vennes et al. 1997; (8) Liebert et al. 2005a; (9) Limoges et al. 2009; (10) Dreizler \& Werner 1996; (11) Farihi 2009; (12) Zuckerman et al. 2003; (13) Farihi et al. 2005a; (14) Hutchings et al. 1996; (15) Eggen \& Greenstein 1965; (16) Liebert et al. 2003; (17) Silvestri et al. 2006; (18) Wagner et al. 1986; (19) Dahn et al. 1988; (20) Greenstein 1976; (21) Hügelmeyer et al. 2006; (22) Kilkenny et al. 1997; (23) Castanheira et al. 2006; (24) Dahn et al. 1982; (25) Kilic et al. 2006; (26) Tappert et al. 2009; (27) Liebert et al. 1994; (28) Kawka et al. 2004; (29) Bergeron et al. 1992; (30) Oswalt et al. 1984.

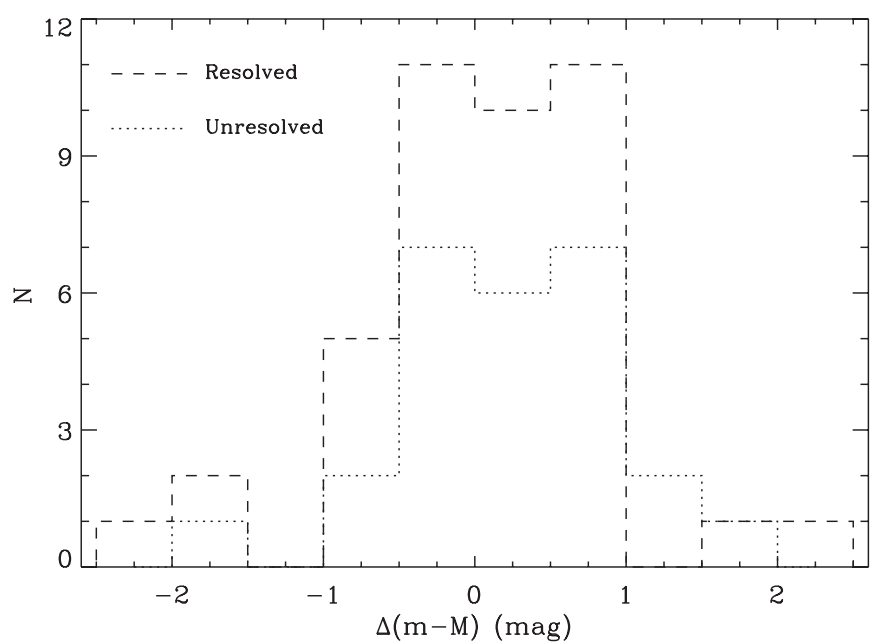

Figure 4. Histogram of the difference in distance moduli between the white dwarf and $\mathrm{M}$ dwarf components of all such binaries.

There are four distinct types of photometric distance mismatches and each is worth brief mention.

1. $\Delta(m-M)>+0.5$ mag visual binaries: 14 systems. These may be explained if the widely separated $\mathrm{M}$ dwarf is itself double or the white dwarf luminosity is overestimated (higher mass or lower temperature); the latter is plausible given the optical spectroscopic contamination by the companion. 
Table 4

Parameters of Resolved Secondary and Tertiary Stars

\begin{tabular}{|c|c|c|c|c|c|}
\hline Primary & Companion & SpT & $I-K$ & $\begin{array}{l}d_{\mathrm{rd}} \\
(\mathrm{pc})\end{array}$ & References \\
\hline $0034-211$ & LTT 0329B & dM3 & 2.15 & 75 & 1,2 \\
\hline $0116-231$ & GD 695B & $\mathrm{dM} 4$ & 2.35 & 166 & $1,3,4$ \\
\hline $0131-163$ & GD 984B & $\mathrm{dM} 3.5$ & 2.26 & 109 & 1,5 \\
\hline $0145-257$ & GD 1401B & $\mathrm{dM} 3.5$ & 2.28 & 80 & $1,6,7$ \\
\hline $0205+133$ & PG $0205+133 B$ & $\mathrm{dM} 2$ & 1.99 & 157 & $1,3,8$ \\
\hline $0208-153$ & МСТ 0208-1520B & $\mathrm{dM} 2.5$ & 2.06 & 121 & 1,3 \\
\hline $0219+282$ & KUV 02196+2816B & dM5 & 2.85 & 324 & 1,3 \\
\hline $0237+115$ & PG $0237+115 B$ & $\mathrm{dM} 2.5$ & 2.13 & 207 & $1,8,9$ \\
\hline $0257-005$ & KUV 02579-0036B & $\mathrm{dM} 4.5$ & 2.56 & 494 & $1,3,10$ \\
\hline $0309-275$ & WD 0309-275B & $\mathrm{dM} 0$ & 1.87 & 339 & 1,3 \\
\hline \multirow[t]{2}{*}{$0324+738^{a}$} & G221-11A & dM5 & 2.68 & 40 & 1 \\
\hline & G221-10B & dM6 & 3.12 & 40 & 1 \\
\hline $0347-137$ & GD 51B & $\mathrm{dM} 4$ & 2.33 & 51 & $1,5,11$ \\
\hline $0357-233$ & Ton S 392B & dM3 & 2.17 & 414 & $1,3,11$ \\
\hline $0430+136$ & KUV 04304+1339B & $\mathrm{dM} 4$ & 2.35 & 103 & 1,3 \\
\hline \multirow{2}{*}{$0518+333^{\mathrm{a}}$} & G86-B1A & dM3 & 2.14 & 65 & 1 \\
\hline & G86-B1C & dM3 & 2.17 & 65 & 1 \\
\hline \multirow[t]{2}{*}{$0824+288$} & PG 0824+288B & $\mathrm{dC}$ & 2.05 & & 1,12 \\
\hline & PG $0824+288 \mathrm{C}$ & $\mathrm{dM} 2$ & 2.06 & 195 & 1,11 \\
\hline $0915+201$ & LB 3016B & dM3 & 2.20 & 550 & 1,3 \\
\hline $0933+025$ & PG $0933+025 B$ & $\mathrm{dM} 4$ & 2.32 & 87 & $1,5,11$ \\
\hline \multirow[t]{2}{*}{$0949+451$} & HS 0949+4508B & $\mathrm{dM} 4.5$ & 2.46 & 58 & 1,9 \\
\hline & HS $0949+4508 \mathrm{C}$ & dM5 & 2.85 & 72 & 1 \\
\hline $1015-173$ & EC 10150-1722B & dM3 & 2.14 & 459 & 1,3 \\
\hline $1033+464$ & GD 123B & dM5 & 2.68 & 59 & $1,5,11$ \\
\hline $1049+103$ & PG 1049+103B & $\mathrm{dM} 4.5$ & 2.46 & 91 & 1,11 \\
\hline $1106+316$ & Ton 58B & $\mathrm{dM} 2.5$ & 2.12 & 404 & 1,3 \\
\hline $1108+325$ & Ton $60 \mathrm{~B}$ & $\mathrm{dM} 2.5$ & 2.12 & 597 & 1,3 \\
\hline $1133+489$ & PG $1133+489 B$ & dM5 & 2.64 & 344 & 1 \\
\hline $1156+129$ & WD $1156+129 B$ & $\mathrm{dM} 4$ & 2.36 & 169 & 1,3 \\
\hline $1210+464$ & PG 1210+464B & $\mathrm{dM} 2$ & 1.99 & 108 & $1,5,11$ \\
\hline $1218+497$ & PG 1218+497B & dM4 & 2.34 & 165 & 1,3 \\
\hline $1236-004$ & WD 1236-004B & $\mathrm{dM} 2.5$ & 2.13 & 773 & 1,3 \\
\hline $1307-141$ & EC 13077-1411B & $\mathrm{dM} 4$ & 2.37 & 112 & 1,3 \\
\hline $1333+487$ & GD 325B & $\mathrm{dM} 4$ & 2.30 & 34 & $1,2,13$ \\
\hline $1412-049$ & PG 1412-049B & dM0 & 1.81 & 377 & 1,3 \\
\hline \multirow[t]{2}{*}{$1419+576$} & SBS $1419+576 B$ & $\mathrm{dM} 2$ & 2.00 & 374 & 1,14 \\
\hline & SBS $1419+576 \mathrm{C}$ & $\mathrm{dM} 2$ & 2.02 & 378 & 1 \\
\hline $1435+370$ & CBS 194B & $\mathrm{dM} 2.5$ & 2.09 & 192 & 1,3 \\
\hline $1443+346$ & PG $1443+346 B$ & $\mathrm{dM} 2$ & 2.02 & 326 & 1,3 \\
\hline $1502+349$ & CBS 223B & dM5 & 2.78 & 198 & 1,3 \\
\hline $1558+616$ & HS $1558+6140 \mathrm{~B}$ & $\mathrm{dM} 4.5$ & 2.40 & 135 & 1,3 \\
\hline \multirow[t]{2}{*}{$1619+525$} & PG 1619+525B & $\mathrm{dM} 2$ & 1.85 & 291 & 1,3 \\
\hline & PG $1619+525 \mathrm{C}$ & dM5 & 2.62 & 297 & 1,3 \\
\hline $1619+414$ & KUV 16195+4125B & dM5 & 2.76 & 106 & 1,3 \\
\hline $1622+323$ & PG $1622+323 B$ & $\mathrm{dM} 2$ & 2.01 & 365 & 1,3 \\
\hline \multirow[t]{2}{*}{$1631+781$} & RX J1629.0+7804B & dM3 & 2.14 & 73 & $1,5,15$ \\
\hline & RX J1629.0+7804C & $\mathrm{dM} 3.5$ & 2.27 & 72 & 1 \\
\hline $1643+143$ & PG $1643+143 B$ & $\mathrm{dM} 2$ & 1.98 & 156 & $1,5,11$ \\
\hline $1646+062$ & PG 1646+062B & $\mathrm{dM} 3.5$ & 2.22 & 168 & 1 \\
\hline \multirow[t]{2}{*}{$1833+644$} & KUV $18332+6429 B$ & $\mathrm{dM} 3$ & 2.17 & 249 & 1,9 \\
\hline & KUV $18332+6429 \mathrm{C}$ & dM6.5 & 3.2 & 249 & 1 \\
\hline $2151-015$ & LTT 8747B & $\mathrm{dM} 7.5$ & 3.82 & 22 & $1,5,11$ \\
\hline
\end{tabular}

Notes.

a These stars are common proper motion companions which were serendipitously resolved into two components, the entries are secondary and tertiary companions to their respective white dwarfs.

References. (1) This work and Paper I; (2) Probst 1983; (3) Wachter et al. 2003; (4) Lamontagne et al. 2000; (5) Schultz et al. 1996; (6) Green et al. 2000; (7) Finley et al. 1997; (8) Green et al. 1986; (9) Hoard et al. 2007; (10) Farihi 2009; (11) Farihi et al. 2005a; (12) Heber et al. 1993; (13) Greenstein 1974; (14) Stepanian et al. 2001; (15) Cooke et al. 1992.
Table 5

Parameters of Unresolved Secondary Stars

\begin{tabular}{|c|c|c|c|c|c|}
\hline Primary & Companion & SpT & $I-K$ & $\begin{array}{c}d_{\mathrm{rd}} \\
(\mathrm{pc})\end{array}$ & References \\
\hline $0023+388$ & G171-B10C & dM5.5 & 3.02 & 70 & $1,2,3$ \\
\hline $0145-221$ & GD 1400B & dL6.5 & 5.8 & 35 & $1,2,4,5,6$ \\
\hline $0303-007$ & KUV 03036-0043B & dM4 & 2.32 & 84 & $1,2,7$ \\
\hline $0331-356$ & HE $0331-3541 B$ & $\mathrm{dM} 2.5$ & 2.08 & 77 & 1,8 \\
\hline $0354+463$ & Rubin $80 \mathrm{~B}$ & $\mathrm{dM} 7$ & 3.63 & 44 & $1,9,10$ \\
\hline $0430+136^{\mathrm{a}}$ & KUV 04304+1339C & dM5.5 & 2.82 & 151 & 1 \\
\hline $0458-665$ & RX J0458.9-6628B & dM3 & 2.15 & 176 & $1,2,11$ \\
\hline $0752-146$ & LTT 2980B & dM6 & 3.07 & 33 & $1,9,10$ \\
\hline $0812+478$ & PG 0812+478B & $\mathrm{dM} 4$ & 2.33 & 174 & 1,2 \\
\hline $0908+226$ & LP 369-15B & dM3 & 2.20 & 425 & 1,2 \\
\hline $1001+203$ & Ton $1150 \mathrm{~B}$ & $\mathrm{dM} 2.5$ & 2.13 & 123 & $1,9,10$ \\
\hline $1026+002$ & PG 1026+002B & $\mathrm{dM} 4.5$ & 2.50 & 45 & $1,9,10,12$ \\
\hline $1037+512$ & PG $1037+512 B$ & $\mathrm{dM} 4$ & 2.37 & 110 & 1,2 \\
\hline $1051+516$ & SBS $1051+516 B$ & dM3 & 2.16 & 183 & $1,13,14$ \\
\hline $1133+358$ & G147-65B & $\mathrm{dM} 4.5$ & 2.55 & 50 & $1,15,16$ \\
\hline $1140+004$ & WD $1140+004 B$ & $\mathrm{dM} 4.5$ & 2.42 & 265 & 1,17 \\
\hline $1247-176$ & EC 12477-1738B & $\mathrm{dM} 4.5$ & 2.46 & 95 & $1,2,8$ \\
\hline $1333+487^{\mathrm{a}}$ & GD $325 \mathrm{C}$ & dM6.5 & 3.28 & 34 & 1 \\
\hline $1333+005$ & LP 618-14B & $\mathrm{dM} 4.5$ & 2.39 & 148 & 1,9 \\
\hline $1334-326$ & EC 13349-3237B & dM0 & 1.88 & 432 & 1,18 \\
\hline $1339+606$ & SBS 1339+606B & $\mathrm{dM} 3.5$ & 2.25 & 347 & $1,19,20$ \\
\hline $1433+358$ & GD 337B & $\mathrm{dM} 4.5$ & 2.49 & 181 & 1,15 \\
\hline $1436-216$ & EC 14363-2137B & $\mathrm{dM} 2.5$ & 2.11 & 173 & $1,2,21$ \\
\hline $1458+171$ & PG $1458+171 B$ & $\mathrm{dM} 4.5$ & 2.57 & 177 & 1,2 \\
\hline $1504+546$ & CBS 301B & dM3 & 2.16 & 223 & $1,2,13$ \\
\hline $1517+502$ & CBS 311B & $\mathrm{dC}$ & 2.80 & $\ldots$ & $1,2,22$ \\
\hline $1522+508$ & CBS 318B & $\mathrm{dM} 3.5$ & 2.23 & 294 & $1,2,14$ \\
\hline $1717-345$ & WD 1717-345B & $\mathrm{dM} 4$ & 2.34 & 68 & 1,23 \\
\hline $2009+622$ & GD 543B & $\mathrm{dM} 4$ & 2.34 & 151 & 1,10 \\
\hline $2257+162$ & PG 2257+162B & $\mathrm{dM} 4.5$ & 2.52 & 275 & 1,2 \\
\hline $2317+268$ & KUV 23176+2650B & $\mathrm{dM} 3.5$ & 2.24 & 216 & 1,2 \\
\hline
\end{tabular}

Notes. All of the binary systems represented by the table entries should be radial velocity variables, some of which have been confirmed since the ACS targets were selected. Additionally, the white dwarf primaries may be polluted by wind from the companions, as in 0354+463 and 1026+002.

a These tentatively identified tertiary companions were singled out on the basis of clear photometric excess in the F814W bandpass, and overly wide PSFs, but require spectroscopic confirmation.

References. (1) This work and Paper I; (2) Wachter et al. 2003; (3) Reid 1996; (4) Farihi \& Christopher 2004; (5) Farihi et al. 2005b; (6) Dobbie et al. 2005; (7) Wegner et al. 1987; (8) Koester et al. 2001; (9) Farihi et al. 2005a; (10) Schultz et al. 1996; (11) Hutchings et al. 1996; (12) Saffer et al. 1993; (13) Stepanian et al. 2001; (14) Silvestri et al. 2006; (15) Probst 1983; (16) Greenstein 1976; (17) Raymond et al. 2003; (18) Kilkenny et al. 1997; (19) Fleming et al. 1996; (20) Hoard et al. 2007; (21) Zuckerman et al. 2003; (22) Liebert et al. 1994; (23) Reid et al. 1988.

Table 6

Three Very Close (Visual) Double M Dwarfs

\begin{tabular}{lcrrr}
\hline \hline Triple System & $\begin{array}{c}\alpha \\
(\operatorname{arcsec})\end{array}$ & \multicolumn{1}{c}{$\begin{array}{c}\text { P.A. } \\
(\mathrm{deg})\end{array}$} & \multicolumn{1}{c}{$\begin{array}{c}I_{B} \\
(\mathrm{mag})\end{array}$} & \multicolumn{1}{c}{$\begin{array}{c}I_{C} \\
(\mathrm{mag})\end{array}$} \\
\hline $0949+451$ & $0.038 \pm 0.004$ & $170.1 \pm 1.4$ & $14.00 \pm 0.08$ & $14.99 \pm 0.19$ \\
$1419+576$ & $0.030 \pm 0.005$ & $22.0 \pm 3.1$ & $15.86 \pm 0.13$ & $15.89 \pm 0.13$ \\
$1631+781$ & $0.029 \pm 0.005$ & $15.4 \pm 3.3$ & $12.76 \pm 0.09$ & $13.67 \pm 0.13$ \\
\hline
\end{tabular}

2. $\Delta(m-M)>+0.5$ mag unresolved doubles: 12 systems. Similar to type (1), but binarity in the M dwarf component itself (i.e., a spatially unresolved triple system) is unlikely.

3. $\Delta(m-M)<-0.5$ mag visual binaries: six systems. Somewhat puzzling, these cases suggest the white dwarf has an underestimated luminosity and may be binary or 


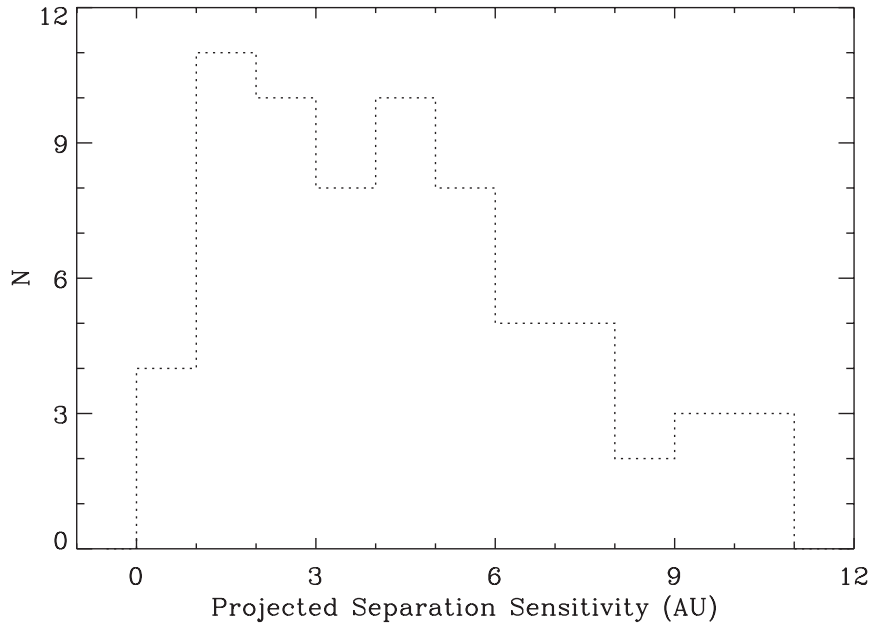

Figure 5. Histogram of minimum projected separations to which this study was sensitive for all white dwarf-M dwarf binaries, assuming an angular separation of a single ACS HRC pixel of $0{ }^{\prime} .025$.

hotter than current estimates. A somewhat underluminous $\mathrm{M}$ dwarf is also possible.

4. $\Delta(m-M)<-0.5$ mag unresolved doubles: three systems. Similar to (3), a relatively low mass white dwarf can account for this type of mismatch, which is a realistic possibility for these post-common envelope systems.

\subsection{Distribution of Projected Orbital Separations}

All double star parameters are listed in Table 7, and triples are listed once for each unique pair. The adopted distance to the system was taken to be the average of the white dwarf and $\mathrm{M}$ dwarf photometric distances. Column 8 lists the projected separation in AU for all visual doubles, which is actually the minimum value for the binary semimajor axis (for zero inclination). Column 9 lists an upper limit to the binary semimajor axis for all spatially unresolved doubles, assuming an angular separation less than $0{ }^{\prime} .025$ or 1 ACS HRC pixel; given the three detections in Table 6 (see also Section 3.1), this is likely to be a safe assumption.

Figure 5 plots a histogram of this limiting 1 pixel resolution multiplied with the adopted distance for all 72 white dwarfs with low-mass companions. From this plot and Table 7, it is clear that the observations were sufficiently sensitive to detect binaries with semimajor axes down to 1-2 AU; the Table 6 double $\mathrm{M}$ dwarfs have projected separations ranging from 2 to 9 AU.

Figure 6 plots histograms of the minimum and maximum orbital separations for all double components within these 72 white dwarf-red dwarf systems. This is the main result of this work: there is essentially no overlap between these two populations and the region between a few to one dozen AU is empty for low-mass companions to white dwarfs. While the displayed histograms overlap slightly due to the plot resolution, they represent minimum and maximum values for orbital separations, and hence any overlap does not indicate that the two populations occupy the same region. The actual values, for which the unresolved systems would shift to smaller separations and the resolved systems would shift to larger separations, almost certainly do not overlap by any significant amount.

To further test this conclusion, Monte Carlo simulations were performed. A sample of 10,000 binaries were randomly selected from a population with the following characteristics: (1) a

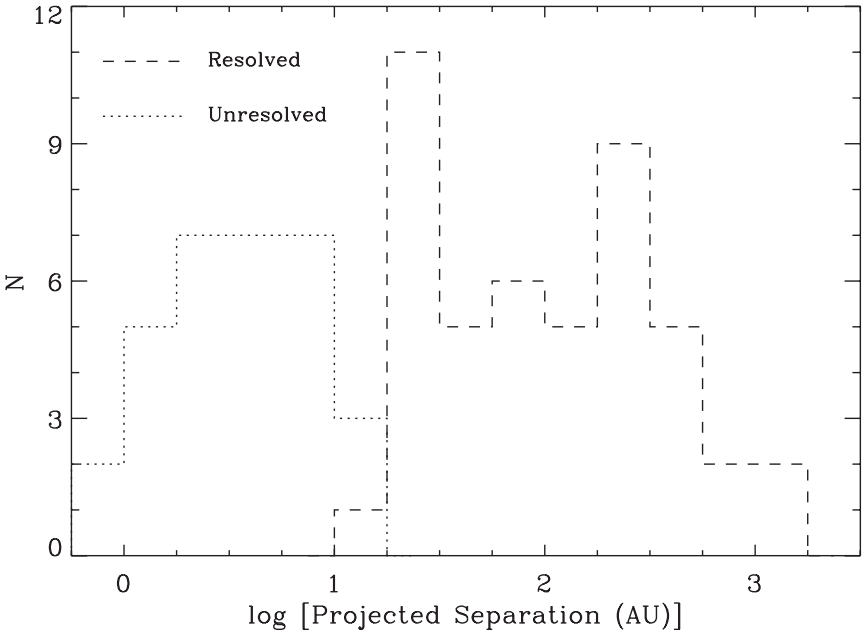

Figure 6. Histogram of spatially resolved (minimum, dashed line) and spatially unresolved (maximum, dotted line) orbital separations for all 72 white dwarf-M dwarf binary and triple systems in the study. The spatially unresolved pairs were assumed to have separations less than a single ACS HRC pixel of 0.'025.

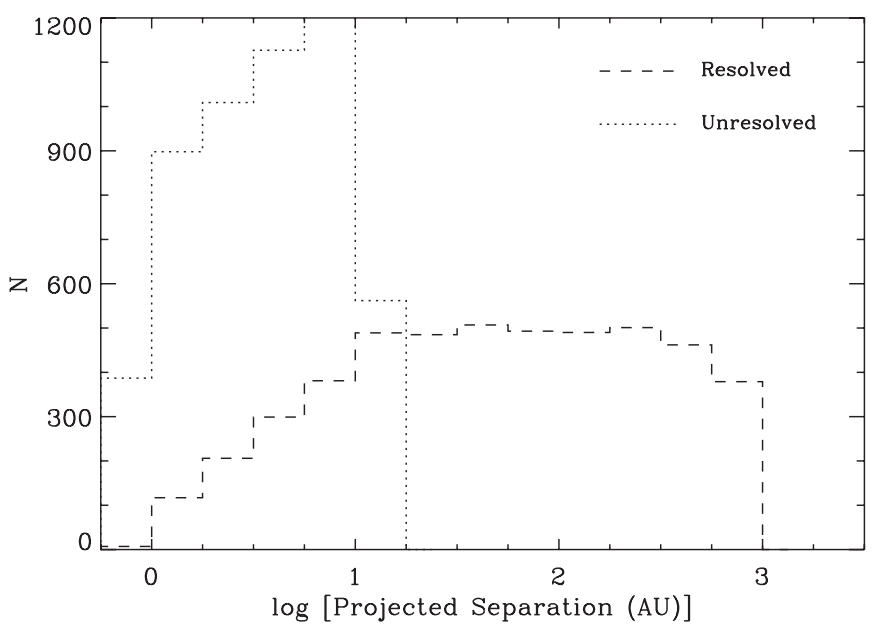

Figure 7. Same as Figure 6 but for a Monte Carlo simulated population of 10,000 binaries with a uniform distribution of orbital separations (see Section 3.7 for details). For each simulated binary, if the projected angle on the sky is less than a single ACS HRC pixel, then the system is counted as spatially unresolved, and plotted with an upper limit to its actual separation. If the angular separation is greater than or equal to 0.025 , the binary is counted as spatially resolved and plotted with this lower limit to its actual semimajor axis. The simulations clearly demonstrate that binaries in the few to several AU range were detectable in the ACS study. A K-S test supports the conclusion that the actual data exhibit a bimodal distribution in semimajor axis at $99.95 \%$ confidence.

logarithmically uniform distribution of semimajor axes between 0.01 and $1000 \mathrm{AU},(2)$ a logarithmically uniform distribution of distances from the Sun between 30 and $500 \mathrm{AU}$, (3) a uniform distribution of orbital inclinations with respect to the plane of the sky, and (4) a uniform distribution of orbital phases within the binary reference frame. For simplicity, the orbits were assumed to be circular. Each randomly selected binary was projected onto the plane of the sky and its angular separation was calculated. If this angle was smaller than 1 ACS HRC pixel, the binary was deemed to be spatially unresolved and an upper limit to the orbital separation was calculated as above. If instead the angle on the sky was greater than or equal to $00^{\prime} 025$, the binary was declared to be spatially resolved and a projected separation was calculated. The results of the simulations are plotted in Figure 7 in precisely the same manner as the actual data in Figure 6. 
Table 7

Parameters for All Double Stars

\begin{tabular}{|c|c|c|c|c|c|c|c|c|c|c|}
\hline $\begin{array}{l}\text { White Dwarf } \\
\text { System }\end{array}$ & $\begin{array}{c}\text { Binary } \\
\text { Components }\end{array}$ & $\begin{array}{c}\alpha^{\mathrm{a}} \\
(\operatorname{arcsec})\end{array}$ & $\begin{array}{l}d_{\mathrm{wd}} \\
(\mathrm{pc})\end{array}$ & $\begin{array}{c}d_{\mathrm{rd}} \\
(\mathrm{pc})\end{array}$ & $\begin{array}{c}\Delta(m-M) \\
(\mathrm{mag})\end{array}$ & $\begin{array}{c}d_{\text {adopt }} \\
(\mathrm{pc})\end{array}$ & $\begin{array}{c}\text { Minimum } \\
\text { Separation } \\
\text { (AU) }\end{array}$ & $\begin{array}{c}\text { Maximum } \\
\text { Separation } \\
(\mathrm{AU})\end{array}$ & $\begin{array}{l}\text { Binary } \\
\text { Type }\end{array}$ & $\begin{array}{c}\text { System } \\
\text { Notes }\end{array}$ \\
\hline \multirow[t]{2}{*}{$0023+388$} & $\mathrm{AB}$ & 24.6 & 59 & 43 & +0.67 & 65 & 1600 & $\ldots$ & $\mathrm{DA}+\mathrm{dM}$ & 1 \\
\hline & $\mathrm{AC}$ & $\ldots$ & 59 & 70 & -0.36 & 65 & $\ldots$ & 1.6 & $\mathrm{DA}+\mathrm{dMe}$ & 6 \\
\hline $0034-211$ & $\mathrm{AB}$ & 0.327 & 61 & 75 & -0.44 & 68 & 22 & $\ldots$ & $\mathrm{DA}+\mathrm{dMe}$ & 5 \\
\hline $0116-231$ & $\mathrm{AB}$ & 1.106 & 164 & 166 & -0.02 & 165 & 182 & $\ldots$ & $\mathrm{DA}+\mathrm{dM}$ & \\
\hline $0131-163$ & $\mathrm{AB}$ & 0.190 & 105 & 109 & -0.09 & 107 & 20 & $\ldots$ & $\mathrm{DA}+\mathrm{dM}$ & \\
\hline $0145-221$ & $\mathrm{AB}$ & $\ldots$ & 43 & $\ldots$ & $\ldots$ & 43 & $\ldots$ & 1.1 & $\mathrm{DA}+\mathrm{dL}$ & 7 \\
\hline $0145+257$ & $\mathrm{AB}$ & 2.294 & 80 & 80 & +0.01 & 80 & 184 & $\ldots$ & $\mathrm{DA}+\mathrm{dM}$ & \\
\hline $0205+133$ & $\mathrm{AB}$ & 1.254 & 223 & 157 & +0.76 & 190 & 238 & $\ldots$ & $\mathrm{DA}+\mathrm{dM}$ & 1 \\
\hline $0208-153$ & $\mathrm{AB}$ & 2.648 & 101 & 121 & -0.39 & 111 & 294 & $\ldots$ & $\mathrm{DA}+\mathrm{dM}$ & \\
\hline \multirow[t]{2}{*}{$0219+282$} & $\mathrm{AB}$ & $\ldots$ & 310 & $\ldots$ & -0.09 & 317 & $\ldots$ & 7.9 & $\mathrm{DA}+\mathrm{DB}$ & 6 \\
\hline & $\mathrm{AC}$ & 0.116 & 310 & 324 & -0.09 & 317 & 37 & $\ldots$ & $\mathrm{DA}+\mathrm{dM}$ & \\
\hline $0237+115$ & $\mathrm{AB}$ & 0.124 & 273 & 207 & +0.59 & 240 & 30 & $\ldots$ & $\mathrm{DO}+\mathrm{dM}$ & 1 \\
\hline $0257-005$ & $\mathrm{AB}$ & 0.978 & 1040 & 490 & +1.61 & 765 & 748 & $\ldots$ & $\mathrm{DAO}+\mathrm{dM}$ & 1 \\
\hline $0303-007$ & $\mathrm{AB}$ & $\ldots$ & 144 & 84 & +1.17 & 114 & $\ldots$ & 2.9 & $\mathrm{DA}+\mathrm{dMe}$ & 2,6 \\
\hline $0309-275$ & $\mathrm{AB}$ & 0.099 & 292 & 339 & -0.32 & 316 & 31 & $\ldots$ & $\mathrm{DA}+\mathrm{dM}$ & \\
\hline \multirow[t]{2}{*}{$0324+738$} & $\mathrm{AB}$ & 12.75 & 40 & 40 & $\ldots$ & 40 & 510 & $\ldots$ & $\mathrm{DC}+\mathrm{dM}$ & 8 \\
\hline & $\mathrm{BC}$ & 0.297 & 40 & 40 & $\ldots$ & 40 & 12 & $\ldots$ & $\mathrm{dM}+\mathrm{dM}$ & 8 \\
\hline $0331-356$ & $\mathrm{AB}$ & $\ldots$ & 141 & 77 & +1.31 & 109 & $\ldots$ & 2.7 & $\mathrm{DA}+\mathrm{dMe}$ & 2,6 \\
\hline $0347-137$ & $\mathrm{AB}$ & 1.052 & 66 & 51 & +0.57 & 59 & 62 & $\ldots$ & $\mathrm{DA}+\mathrm{dMe}$ & 1,5 \\
\hline $0354+463$ & $\mathrm{AB}$ & $\ldots$ & 32 & 44 & -0.67 & 38 & $\ldots$ & 1.0 & $\mathrm{DAZ}+\mathrm{dMe}$ & 4,6 \\
\hline $0357-233$ & $\mathrm{AB}$ & 1.191 & 199 & 414 & -1.60 & 307 & 365 & $\ldots$ & $\mathrm{DA}+\mathrm{dM}$ & 3 \\
\hline \multirow[t]{2}{*}{$0430+136$} & $\mathrm{AB}$ & 0.260 & 376 & 103 & +2.82 & 236 & 61 & $\ldots$ & $\mathrm{DA}+\mathrm{dMe}$ & 1 \\
\hline & AC: & $\ldots$ & 376 & 151 & +1.99 & 236 & $\ldots$ & 5.9 & $\mathrm{DA}+\mathrm{dMe}$ & 2,6 \\
\hline $0458-665$ & $\mathrm{AB}$ & $\ldots$ & 258 & 176 & +0.83 & 217 & $\ldots$ & 5.4 & $\mathrm{DA}+\mathrm{dMe}$ & 2,6 \\
\hline \multirow[t]{2}{*}{$0518+333$} & $\mathrm{AB}$ & 7.475 & 65 & 65 & $\ldots$ & 65 & 486 & $\ldots$ & $\mathrm{DA}+\mathrm{dM}$ & 8 \\
\hline & $\mathrm{BC}$ & 0.158 & 65 & 65 & $\ldots$ & 65 & 10 & $\ldots$ & $\mathrm{dM}+\mathrm{dM}$ & 8 \\
\hline $0752-146$ & $\mathrm{AB}$ & $\ldots$ & 35 & 33 & +0.10 & 34 & $\ldots$ & 0.9 & $\mathrm{DA}+\mathrm{dMe}$ & 6 \\
\hline $0812+478$ & $\mathrm{AB}$ & $\ldots$ & 259 & 174 & +0.86 & 217 & $\ldots$ & 5.4 & $\mathrm{DA}+\mathrm{dM}$ & 2,6 \\
\hline \multirow[t]{2}{*}{$0824+288$} & $\mathrm{AB}$ & 3.330 & 168 & 195 & -0.32 & 182 & 604 & $\ldots$ & $\mathrm{DA}+\mathrm{dM}$ & \\
\hline & $\mathrm{AC}$ & 0.077 & 168 & $\ldots$ & $\ldots$ & 182 & 14 & $\ldots$ & $\mathrm{DA}+\mathrm{dCe}$ & 5 \\
\hline $0908+226$ & $\mathrm{AB}$ & $\ldots$ & 402 & 425 & -0.12 & 414 & $\ldots$ & 10 & $\mathrm{DA}+\mathrm{dMe}$ & 6 \\
\hline $0915+201$ & $\mathrm{AB}$ & 2.312 & 643 & 550 & +0.34 & 597 & 1380 & $\ldots$ & $\mathrm{DA}+\mathrm{dM}$ & \\
\hline $0933+025$ & $\mathrm{AB}$ & 1.232 & 135 & 87 & +0.95 & 111 & 137 & $\ldots$ & $\mathrm{DA}+\mathrm{dMe}$ & 1,5 \\
\hline \multirow[t]{2}{*}{$0949+451$} & $\mathrm{AB}$ & 2.892 & 65 & 65 & +0.01 & 65 & 188 & $\ldots$ & $\mathrm{DA}+\mathrm{dM}$ & \\
\hline & $\mathrm{BC}$ & 0.038 & $\ldots$ & 65 & +0.01 & 65 & 2.5 & $\ldots$ & $\mathrm{dM}+\mathrm{dM}$ & \\
\hline $1001+203$ & $\mathrm{AB}$ & $\ldots$ & 117 & 123 & -0.10 & 120 & $\ldots$ & 3.0 & $\mathrm{DA}+\mathrm{dMe}$ & 6 \\
\hline $1015-173$ & $\mathrm{AB}$ & 0.060 & 226 & 459 & -1.53 & 343 & 21 & $\ldots$ & $\mathrm{DA}+\mathrm{dM}$ & 3 \\
\hline $1026+002$ & $\mathrm{AB}$ & $\ldots$ & 40 & 45 & -0.28 & 42 & $\ldots$ & 1.1 & $\mathrm{DAZ}+\mathrm{dMe}$ & 7 \\
\hline $1033+464$ & $\mathrm{AB}$ & 0.731 & 83 & 59 & +0.75 & 71 & 52 & $\ldots$ & $\mathrm{DA}+\mathrm{dMe}$ & 1,5 \\
\hline $1037+512$ & $\mathrm{AB}$ & $\ldots$ & 133 & 110 & +0.40 & 121 & $\ldots$ & 3.0 & $\mathrm{DA}+\mathrm{dM}$ & 6 \\
\hline $1049+103$ & $\mathrm{AB}$ & 0.262 & 112 & 91 & +0.45 & 102 & 27 & $\ldots$ & $\mathrm{DAZ}+\mathrm{dM}$ & \\
\hline $1051+516$ & $\mathrm{AB}$ & $\ldots$ & 225 & 183 & +0.45 & 204 & $\ldots$ & 5.1 & $\mathrm{DA}+\mathrm{dMe}$ & 6 \\
\hline $1106+316$ & $\mathrm{AB}$ & 0.478 & 257 & 404 & -0.98 & 331 & 158 & $\ldots$ & $\mathrm{DA}+\mathrm{dM}$ & 3 \\
\hline $1108+325$ & $\mathrm{AB}$ & 0.167 & 550 & 597 & -0.18 & 574 & 96 & $\ldots$ & $\mathrm{DA}+\mathrm{dM}$ & \\
\hline $1133+489$ & $\mathrm{AB}$ & 0.090 & 384 & 344 & +0.23 & 364 & 33 & $\ldots$ & $\mathrm{DO}+\mathrm{dM}$ & \\
\hline $1133+358$ & $\mathrm{AB}$ & $\ldots$ & 50 & 50 & $\ldots$ & 50 & $\ldots$ & 1.3 & $\mathrm{DC}+\mathrm{dMe}$ & 6,8 \\
\hline $1140+004$ & $\mathrm{AB}$ & $\ldots$ & 278 & 265 & +0.10 & 272 & $\ldots$ & 6.8 & $\mathrm{DA}+\mathrm{dMe}$ & 6 \\
\hline $1156+129$ & $\mathrm{AB}$ & 0.564 & 214 & 169 & +0.51 & 192 & 108 & $\ldots$ & $\mathrm{DA}+\mathrm{dM}$ & 1 \\
\hline $1210+464$ & $\mathrm{AB}$ & 1.043 & 171 & 108 & +0.99 & 140 & 145 & $\ldots$ & $\mathrm{DA}+\mathrm{dMe}$ & 1,5 \\
\hline $1218+497$ & $\mathrm{AB}$ & 0.303 & 250 & 165 & +0.90 & 208 & 63 & $\ldots$ & $\mathrm{DA}+\mathrm{dM}$ & 1 \\
\hline $1236-004$ & $\mathrm{AB}$ & 0.663 & 575 & 773 & -0.64 & 674 & 447 & $\ldots$ & $\mathrm{DA}+\mathrm{dMe}$ & 3,5 \\
\hline $1247-176$ & $\mathrm{AB}$ & $\ldots$ & 141 & 95 & +0.85 & 118 & $\ldots$ & 3.0 & $\mathrm{DA}+\mathrm{dM}$ & 2,7 \\
\hline $1307-141$ & $\mathrm{AB}$ & 2.133 & 158 & 112 & +0.76 & 135 & 288 & $\ldots$ & $\mathrm{DA}+\mathrm{dM}$ & 1 \\
\hline \multirow[t]{2}{*}{$1333+487$} & $\mathrm{AB}$ & 2.950 & 34 & 34 & $\ldots$ & 34 & 100 & $\ldots$ & $\mathrm{DB}+\mathrm{dM}$ & 8 \\
\hline & AC: & $\ldots$ & 34 & 34 & $\ldots$ & 34 & $\ldots$ & 0.9 & $\mathrm{DB}+\mathrm{dM}$ & 6,8 \\
\hline $1333+005$ & $\mathrm{AB}$ & $\ldots$ & 69 & 148 & -1.66 & 109 & $\ldots$ & 2.7 & $\mathrm{DA}+\mathrm{dM}$ & 4,6 \\
\hline $1334-326$ & $\mathrm{AB}$ & $\ldots$ & 619 & 432 & +0.78 & 526 & $\ldots$ & 13 & $\mathrm{DA}+\mathrm{dMe}$ & 2,7 \\
\hline $1339+606$ & $\mathrm{AB}$ & $\ldots$ & 439 & 347 & +0.51 & 393 & $\ldots$ & 9.8 & $\mathrm{DA}+\mathrm{dMe}$ & 2,6 \\
\hline $1412-049$ & $\mathrm{AB}$ & 3.507 & 398 & 377 & +0.12 & 388 & 1360 & $\ldots$ & $\mathrm{DA}+\mathrm{dM}$ & \\
\hline \multirow[t]{2}{*}{$1419+576$} & $\mathrm{AB}$ & 0.655 & 258 & 376 & -0.82 & 317 & 208 & $\ldots$ & $\mathrm{DB}+\mathrm{dM}$ & 2 \\
\hline & $\mathrm{BC}$ & 0.030 & $\ldots$ & 376 & $\ldots$ & 317 & 9.5 & $\ldots$ & $\mathrm{dM}+\mathrm{dM}$ & \\
\hline $1433+538$ & $\mathrm{AB}$ & $\ldots$ & 151 & 181 & -0.39 & 166 & $\ldots$ & 4.2 & $\mathrm{DA}+\mathrm{dM}$ & 6 \\
\hline $1435+370$ & $\mathrm{AB}$ & 1.253 & 129 & 192 & -0.87 & 161 & 201 & $\ldots$ & $\mathrm{DA}+\mathrm{dM}$ & 3 \\
\hline $1436-216$ & $\mathrm{AB}$ & $\ldots$ & 193 & 173 & +0.24 & 183 & $\ldots$ & 4.6 & $\mathrm{DA}+\mathrm{dMe}$ & 6 \\
\hline
\end{tabular}


Table 7

(Continued)

\begin{tabular}{|c|c|c|c|c|c|c|c|c|c|c|}
\hline $\begin{array}{c}\text { White Dwarf } \\
\text { System }\end{array}$ & $\begin{array}{c}\text { Binary } \\
\text { Components }\end{array}$ & $\begin{array}{c}\alpha^{\mathrm{a}} \\
(\operatorname{arcsec})\end{array}$ & $\begin{array}{l}d_{\mathrm{wd}} \\
(\mathrm{pc})\end{array}$ & $\begin{array}{l}d_{\mathrm{rd}} \\
(\mathrm{pc})\end{array}$ & $\begin{array}{c}\Delta(m-M) \\
\quad(\mathrm{mag})\end{array}$ & $\begin{array}{l}d_{\text {adopt }} \\
(\mathrm{pc})\end{array}$ & $\begin{array}{c}\text { Minimum } \\
\text { Separation } \\
(\mathrm{AU})\end{array}$ & $\begin{array}{c}\text { Maximum } \\
\text { Separation } \\
\text { (AU) }\end{array}$ & $\begin{array}{l}\text { Binary } \\
\text { Type }\end{array}$ & $\begin{array}{l}\text { System } \\
\text { Notes }\end{array}$ \\
\hline $1443+336$ & $\mathrm{AB}$ & 0.679 & 239 & 326 & -0.68 & 283 & 192 & $\ldots$ & $\mathrm{DA}+\mathrm{dM}$ & 3 \\
\hline $1458+171$ & $\mathrm{AB}$ & $\ldots$ & 226 & 177 & +0.53 & 202 & $\ldots$ & 5.0 & $\mathrm{DA}+\mathrm{dMe}$ & 2,6 \\
\hline $1502+349$ & $\mathrm{AB}$ & 1.912 & 161 & 198 & -0.46 & 180 & 343 & $\ldots$ & $\mathrm{DA}+\mathrm{dM}$ & \\
\hline $1504+546$ & $\mathrm{AB}$ & $\ldots$ & 243 & 223 & +0.19 & 233 & $\ldots$ & 5.8 & $\mathrm{DA}+\mathrm{dMe}$ & 6 \\
\hline $1517+502$ & $\mathrm{AB}$ & $\cdots$ & 406 & $\ldots$ & $\ldots$ & 406 & $\ldots$ & 10 & $\mathrm{DA}+\mathrm{dCe}$ & 6 \\
\hline $1522+508$ & $\mathrm{AB}$ & $\ldots$ & 238 & 294 & -0.46 & 266 & $\ldots$ & 6.7 & $\mathrm{DA}+\mathrm{dMe}$ & 6 \\
\hline $1558+616$ & $\mathrm{AB}$ & 0.719 & 141 & 135 & +0.10 & 138 & 99 & $\ldots$ & $\mathrm{DA}+\mathrm{dM}$ & \\
\hline \multirow[t]{2}{*}{$1619+525$} & $\mathrm{AB}$ & 2.593 & 94 & 291 & -2.46 & 194 & 503 & $\ldots$ & $\mathrm{DA}+\mathrm{dM}$ & 1 \\
\hline & $\mathrm{AC}$ & 0.465 & 94 & 297 & -2.51 & 194 & 90 & $\begin{array}{l}\cdots \\
\ldots\end{array}$ & $\mathrm{DA}+\mathrm{dM}$ & 1 \\
\hline $1619+414$ & $\mathrm{AB}$ & 0.232 & 156 & 106 & +0.83 & 131 & 30 & $\ldots$ & $\mathrm{DA}+\mathrm{dM}$ & 1 \\
\hline $1622+323$ & $\mathrm{AB}$ & 0.093 & 513 & 365 & +0.74 & 439 & 41 & $\ldots$ & $\mathrm{DA}+\mathrm{dM}$ & 1 \\
\hline \multirow[t]{2}{*}{$1631+781$} & $\mathrm{AB}$ & 0.302 & 75 & 73 & +0.07 & 74 & 22 & $\ldots$ & $\mathrm{DA}+\mathrm{dMe}$ & 5 \\
\hline & $\mathrm{BC}$ & 0.029 & $\ldots$ & 73 & $\ldots$ & 74 & 2.1 & $\ldots$ & $\mathrm{dM}+\mathrm{dM}$ & \\
\hline $1643+143$ & $\mathrm{AB}$ & 0.312 & 153 & 156 & -0.05 & 155 & 48 & $\ldots$ & $\mathrm{DA}+\mathrm{dM}$ & \\
\hline $1646+062$ & $\mathrm{AB}$ & 0.161 & 174 & 168 & +0.07 & 171 & 28 & $\ldots$ & $\mathrm{DA}+\mathrm{dM}$ & \\
\hline $1717-345$ & $\mathrm{AB}$ & $\ldots$ & 137 & 68 & +1.53 & 103 & $\ldots$ & 2.6 & $\mathrm{DA}+\mathrm{dMe}$ & 2,6 \\
\hline \multirow[t]{2}{*}{$1833+644$} & $\mathrm{AB}$ & 0.079 & 227 & 249 & -0.21 & 238 & 19 & $\ldots$ & $\mathrm{DA}+\mathrm{dM}$ & \\
\hline & $\mathrm{AC}$ & 1.820 & 227 & $\ldots$ & $\ldots$ & 238 & 429 & $\cdots$ & $\mathrm{DA}+\mathrm{dM}$ & \\
\hline $2009+622$ & $\mathrm{AB}$ & $\ldots$ & 125 & 151 & -0.41 & 138 & $\ldots$ & 3.5 & $\mathrm{DA}+\mathrm{dM}$ & 7 \\
\hline $2151-015$ & $\mathrm{AB}$ & 1.082 & 22 & 22 & +0.04 & 22 & 24 & $\ldots$ & $\mathrm{DA}+\mathrm{dMe}$ & 5 \\
\hline $2257+162$ & $\mathrm{AB}$ & $\begin{array}{l}1.002 \\
\ldots\end{array}$ & 213 & 275 & -0.56 & 244 & $\begin{array}{l}24 \\
\ldots\end{array}$ & 6.1 & $\mathrm{DA}+\mathrm{dM}$ & 4,6 \\
\hline $2317+268$ & $\mathrm{AB}$ & $\ldots$ & 284 & 216 & +0.59 & 250 & $\ldots$ & 6.3 & $\mathrm{DA}+\mathrm{dM}$ & 3,6 \\
\hline
\end{tabular}

Notes. (1) Wide binary with $\Delta(m-M)>+0.5 \mathrm{mag}$; (2) close binary with $\Delta(m-M)>+0.5 \mathrm{mag}$; (3) wide binary with $\Delta(m-M)<-0.5 \mathrm{mag}$; (4) close binary with $\Delta(m-M)<-0.5 \mathrm{mag}$; (5) wide binary with active dMe secondary; (6) radial velocity variable candidate; (7) radial velocity variable with known period (Section 3.6); (8) distance from trigonometric parallax.

${ }^{a}$ For spatially unresolved binaries, an upper limit of $0{ }^{\prime \prime} 025$ was assumed based on the separations of the closest spatially resolved pairs in Table 6.

The simulated data describe a population that is clearly distinct from the observed binary population, despite representing the same (potential) range of semimajor axes, distances from the Sun, and angular detection criterion. A Kolmogorov-Smirnov (K-S) test was performed on the observed and simulated samples of spatially resolved binaries, resulting in a probability of $99.95 \%$ that the two populations are not related. These results demonstrate that the actual population of white dwarf plus low-mass stellar companions does not have a uniform distribution of orbital semimajor axes, but is a population with a deficit of systems with semimajor axes below 20 AU. From radial velocity studies sensitive to the other end of the distribution, the observed population also has a distinct lack of binaries with periods longer than 10 days (Rebassa-Mansergas et al. 2008; Morales-Rueda et al. 2005). Therefore, the HST ACS observations complete an empirical picture of the bimodal semimajor axis distribution among white dwarfs with low-mass, unevolved (stellar or substellar) companions.

\subsection{Companion Sensitivity}

Regarding the sensitivity to spatially resolved companions as a function of angular separation and contrast ratio, the study was designed to be intrinsically robust in the following manner. For the 72 systems where a reliable near-infrared excess is associated with the white dwarf, in all but one instance there is a corresponding, measurable $I$-band excess. In each case, this excess was predicted on the basis of the near-infrared photometry, and hence the choice of the F814W filter to exploit the expected favorable contrast. These 71 systems were either (1) spatially resolved into two or more components and the dM companion was directly detected or (2) measured to have a clear $\left(I_{\mathrm{ACS}}-I_{\mathrm{WD}}>0.5 \mathrm{mag}\right)$ photometric excess indicating the presence of the $\mathrm{dM}$ companion. Thus, among these targets, all previously suspected companions have been directly or indirectly detected; none were missed.

The single exception is $0145-221$ where the $\mathrm{L}$ dwarf companion is too cool to reveal itself via excess emission at F814W (Farihi \& Christopher 2004). However, this system has recently been found to be a short-period binary and is therefore not expected to be directly imaged in the ACS observations (see the Appendix for further details). Apart from this one case, the remaining 71 systems all have $\Delta I<3.0$ mag between the white dwarf and $\mathrm{M}$ dwarf components, with all but a few having contrasts milder than 10:1 in flux. One of the companions to $1833+464$ is readily detected at $0{ }^{\prime \prime} 08$ and $\Delta I=1.8 \mathrm{mag}$, while two of the three close $M$ dwarf pairs in Table 6 have $0^{\prime \prime} 03$ and $\Delta I \approx 1 \mathrm{mag}$.

\subsection{Distribution of Companion Spectral Types}

Figures 8 and 9 plot the relative frequency of $M$ dwarf companions as a function of their $I-K$ color and spectral type inferred from this index. The two populations of spatially resolved and unresolved companions appear generally similar, and agree well with the distribution found by Farihi et al. (2005a) with a peak around M3.5. Interestingly, there appears to be a relative dearth of early $\mathrm{M}$ dwarfs among the spatially unresolved companions. The white dwarf primaries in the sample are not sufficiently well characterized to instill high confidence in their predicted $I$-band fluxes necessary to derive the $I-K$ colors of the spatially unresolved $M$ dwarfs. On the other hand, the distribution of spectral types based on these derivations otherwise matches expectations based on the distribution of 


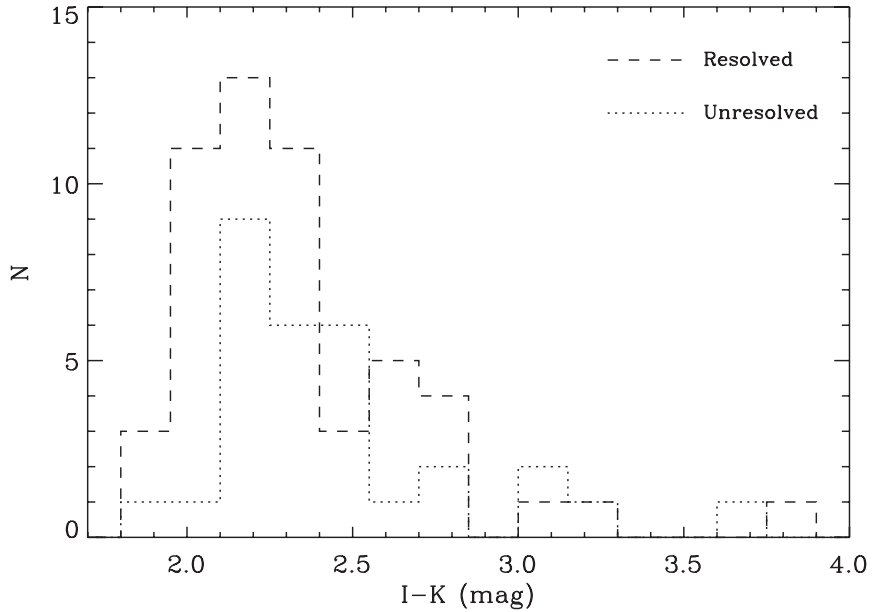

Figure 8. Histogram of the $I-K$ color determinations for all $\mathrm{M}$ dwarf companions observed in the program. The populations are similar except for a relative dearth of the bluest colors among the spatially unresolved companions.

field M dwarfs (Farihi et al. 2005a; Cruz et al. 2003) and that of the spatially resolved companions.

$\mathrm{K}-\mathrm{S}$ tests were employed to compare the cumulative distributions of $I-K$ color and spectral type between the spatially resolved and unresolved systems. The results yielded $P$ values of 0.12 in the $I-K$ color and 0.37 for spectral type, implying the two data sets are most likely distinct. The $P$ value for the spectral type is understandably higher as the raw $I-K$ data have been smoothed during transformation into spectral type. The data are not sufficient to conclude with certainty if the measured difference is the result of small number statistics, errors in deconvolving the $I$-band photometry for spatially unresolved pairs, or real. It is noteworthy that with a similar yet much larger sample of low-mass, main-sequence companions to white dwarfs from the SDSS, Schreiber et al. (2010) find the distribution of spectral types for close (post-common envelope) and wide secondaries to be distinct with high confidence.

Again, the primary deviation between the subsamples here is an apparent deficit of earlier $M$ types among the spatially unresolved secondaries. While some caution is warranted, this putative trend is exactly the opposite of what would be expected if the secondary stars accreted a significant amount of mass during the common envelope phase. This data set is insufficient to rule out or uncover mass transfer at the several to tens of percent level, but it is clear that $0.1-0.2 M_{\odot}$ stars do not emerge from the common envelope with $0.3-0.5 M_{\odot}$. This result emphasizes the fact that mass transfer via Roche lobe overflow from a higher mass to lower mass star is unstable (Paczynski 1976).

\subsection{Confirmed and Candidate Close Binaries}

From these results, and as noted in Table 7, all 29 spatially unresolved binaries are predicted to have short orbital periods; this can be easily tested with radial velocity or photometric monitoring. Some of these systems have additional evidence that favors close binarity, such as variable Balmer line emission, yet none have established periods (Silvestri et al. 2006; Liebert et al. 2005a; Silvestri et al. 2005; Koester et al. 2001; Stepanian et al. 2001; Maxted et al. 2000; Putney 1997; Hutchings et al. 1996; Schultz et al. 1996; Reid 1996). Since 2005 when this study was initiated, 4 of these 29 candidate short-period binaries have been confirmed via independent studies: 0145-221, 1247-176,

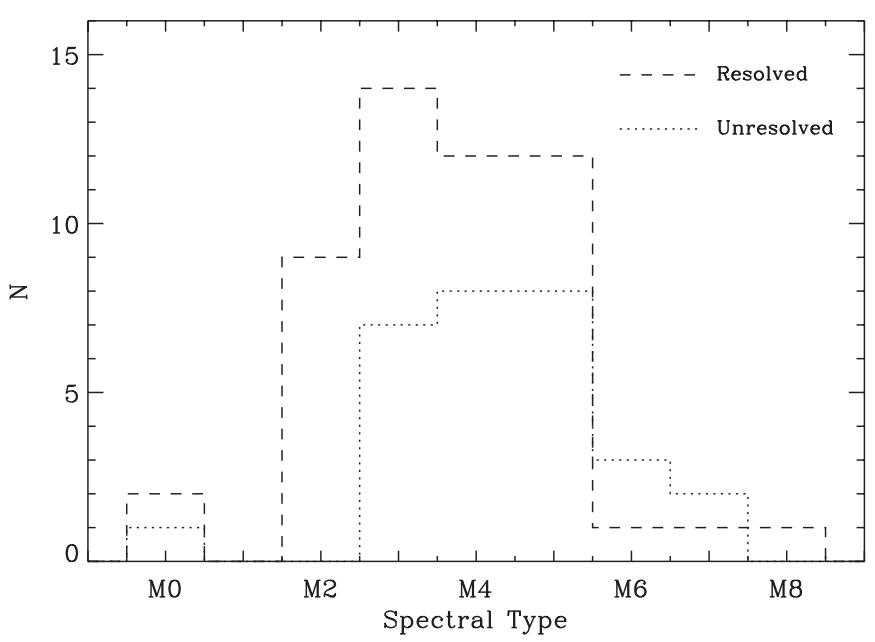

Figure 9. Histogram of $\mathrm{M}$ dwarf companion spectral types inferred from $I-K$ colors. As seen in the previous figure, there are relatively fewer early $M$ dwarfs among the spatially unresolved companions.

1334-326, and 2009+622 (M. R. Burleigh et al. 2010, in preparation; Tappert et al. 2009; Saffer et al. 1993; MoralesRueda et al. 2005). The short-period binary 1026+002 (Saffer et al. 1993) went unrecognized during target selection, and was included in the ACS imaging program. Table 7 lists only those systems with established periods as known close binaries, because variability due to activity is often seen in widely bound systems (nine of these are identified in this study and discussed in the Appendix).

\subsection{Non-binaries and Misidentifications}

Table 8 lists 16 ACS targets that are no longer considered white dwarf-M dwarf binary candidates. These consist of (1) high-risk targets, white dwarfs whose 2MASS data suggested only modest $H$ - or $K_{s}$-band excess (which might have indicated a substellar companion), (2) low-mass stars incorrectly classified as white dwarfs, and (3) white dwarfs co-identified with nearby, bright 2MASS sources (including wide visual companions). Two additional systems $(1156+132,2237-365)$ were not imaged because the white dwarf fell outside of the narrow ACS field of view due to inaccurate coordinates in SIMBAD and the literature. Each of these 18 systems is briefly discussed in Paper I or the Appendix.

\subsection{Two Dwarf Carbon Companions}

Two white dwarfs in this study have low-mass stellar companions which are carbon-enriched counterparts to $\mathrm{M}$ dwarfs: 0824+288 and 1517+502 (Liebert et al. 1994; Heber et al. 1993). The latter binary is spatially unresolved and a strong candidate to have a short orbital period, consistent with the paradigm that dwarf carbon stars are the product of mass transfer from a carbon-rich giant (Dahn et al. 1977). The former, however, is a spatially resolved triple system consisting of a wide $\mathrm{M}$ dwarf secondary and a close visual tertiary. Assuming the tertiary in $0824+288$ is the dwarf carbon star, the lower limit for its semimajor axis is $14 \mathrm{AU}$. Therefore, mass transfer via Roche lobe overflow is effectively ruled out by the ACS observations, presenting something of a challenge to the binary origin of its carbon-polluted exterior. One way to resolve this is to invoke effective wind capture when the white dwarf progenitor star was an asymptotic giant. Prior to the bulk of mass loss during this phase, the binary would have orbited more closely by a factor 
Table 8

Suspected or Confirmed Non-binaries

\begin{tabular}{cccc}
\hline \hline WD & $I-K$ & SpT & References \\
\hline $0324+738$ & +0.25 & DC6 & 1 \\
$0518+333$ & -0.07 & DA5 & 1,2 \\
$0807+190$ & +2.49 & sdK & $1,3,4$ \\
$0937-025$ & +1.57 & sdK5 & 1 \\
$1036-204$ & +1.22 & DQp7 & $1,2,5$ \\
$1106-211$ & +1.78 & dK7 & 1 \\
$1247+550$ & +0.53 & DC12 & 1,6 \\
$1339+346$ & -0.13 & DA3 & 1,2 \\
$1434+289$ & -0.68 & DA2 & 1,2 \\
$1603+125$ & +1.19 & dK2 & 1 \\
$1658+440$ & -0.49 & DA2 & 1,7 \\
$1845+683$ & -0.69 & DA1 & 1,2 \\
$2211+372$ & +0.49 & DC8 & 1,3 \\
$2323+256$ & +0.82 & DA9 & 1,8 \\
$2336-187$ & +0.27 & DA6 & $1,2,9$ \\
$2349-283$ & +0.02 & DA3 & 1,10 \\
\hline
\end{tabular}

Notes. These stars are no longer suspected of having a near-infrared excess consistent with a spatially unresolved low-mass companion within several square arcsec of their position. The stars $0324+738$ and $0518+333$ are part of wide binary systems, but were previously suspected of harboring additional components based on their 2MASS photometry.

References. (1) This work and Paper I; (2) Farihi 2009; (3) Reid \& Gizis 2005; (4) Gizis \& Reid 1997; (5) Liebert et al. 2003; (6) Bergeron et al. 2001; (7) Farihi et al. 2008; (8) Hintzen 1986; (9) Trembley \& Bergeron 2007; (10) Koester et al. 2001.

of a few, putting the dwarf carbon star progenitor as close as 5-7 AU. If the giant envelope extended to a few AU, its wind could potentially pollute a companion orbiting at this semimajor axis.

The dwarf carbon stars were once thought to be a class of halo stars, but these two with white dwarf primaries appear to have disk-like kinematics. Both systems have modest proper motions corresponding to tangential speeds of $40-70 \mathrm{~km} \mathrm{~s}^{-1}$ (Zacharias et al. 2005). The prototype carbon dwarf and halo star G77-61 has very little GALEX near-ultraviolet flux and no detection in the far-ultraviolet, making it unlikely that any putative white dwarf companion (Dearborn et al. 1986) could be warmer than $5000 \mathrm{~K}$.

\subsection{Wind Capture in Close Binaries}

Zuckerman et al. (2003) searched for calcium K absorption lines in the high-resolution spectra of 17 DA white dwarfs with known $\mathrm{M}$ dwarf companions that were spatially unresolved in ground-based observations. Emission from active secondaries prevented a useful search in seven of these cases, and five of the remaining ten white dwarfs were found to be metal polluted (the absorption feature in $1210+464$ is uncertain and this star may not be DAZ; B. Zuckerman 2010, private communication). This result suggests that wind from low-mass stellar companions, when present, can readily pollute the atmosphere of white dwarfs (Debes 2006). Eight of the stars observed by Zuckerman et al. (2003) were imaged with ACS in the current survey.

The binaries $0354+463,0752-146$, and $1026+002$ are all spatially unresolved and therefore likely to be in short-period orbits. In fact, the latter system is a known $14 \mathrm{hr}$ radial velocity variable (Saffer et al. 1993), and contains a DAZ white dwarf. The other two systems should now be considered strong candidates for short-period systems; the first has a DAZ primary

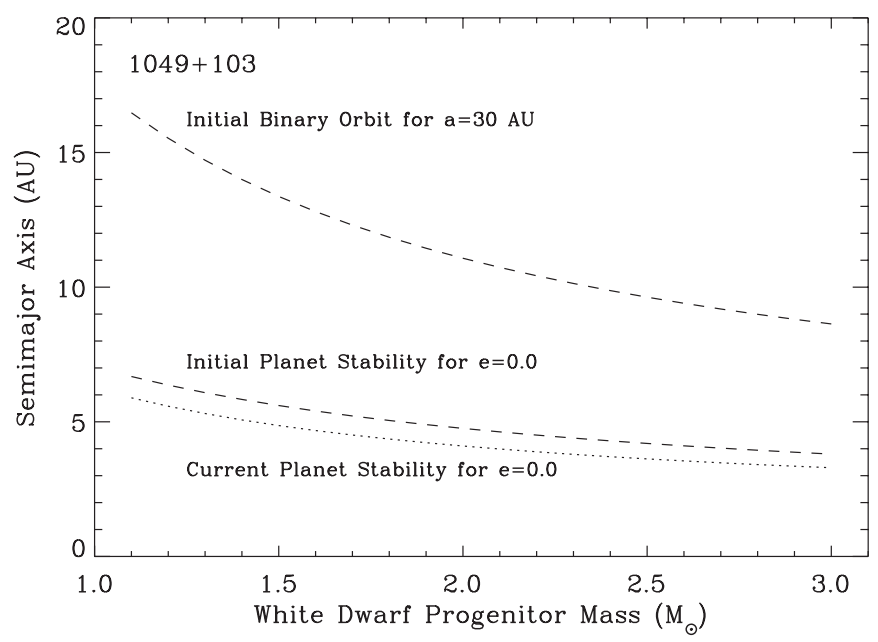

Figure 10. Initial orbit of PG 1049+103 plotted as a function of the white dwarf progenitor mass, using the initial to final mass relationship (Dobbie et al. 2009). Also plotted is the limit for stable planetary orbits (Holman \& Wiegiert 1999) in the initial and current configurations. Because the orbits of any planets initially wider than a few AU would expand by a factor of a few, it is unlikely they would now persist at the white dwarf.

while the second displays prominent calcium emission from the M dwarf and was indeterminate (Zuckerman et al. 2003).

The doubles 0034-211, 0347-137, 0933+025, 1049+103, and $1210+464$ are all spatially resolved and hence have wide orbital separations where wind from the $M$ dwarf cannot effectively pollute the white dwarf. Surprisingly then, 1049+103 is a DAZ white dwarf. There is no evidence of an unseen, closely orbiting third component in this system; this would have to be a very low mass star or brown dwarf but Spitzer studies reveal no evidence for these types of companions at metal-polluted white dwarfs (Farihi et al. 2009). The white dwarf primary is rather warm at $20,600 \mathrm{~K}$ (Liebert et al. 2005a), and is currently accreting metals at $1.1 \times 10^{8} \mathrm{~g} \mathrm{~s}^{-1}$ (Koester 2009), suggesting the likely presence of circumstellar material.

When both components of $1049+103$ were on the main sequence, the orbital separation was smaller by a factor of few, and any surviving planetary system that might pollute the white dwarf would have to be contained within a relatively narrow region (Holman \& Wiegiert 1999). The asymptotic giant predecessor directly engulfed the innermost regions around the white dwarf, while orbits outside its maximum extent (including the orbit of the companion star) expanded by a factor of a few. Also in response to the change in binary mass ratio postmain sequence, the maximum radius for stable planetary orbits decreased. Therefore, the $\mathrm{M}$ dwarf companion currently restricts stable planets at the white dwarf to lie in a region that should be mostly or totally empty. Figure 10 illustrates this; barring changes in eccentricity, there are no stable planetary orbits over the main- and post-main-sequence lifetime of the binary, leaving the nature of the DAZ white dwarf in this system somewhat of a puzzle.

\subsection{Systems with Potential Orbital Motion}

There are 13 systems imaged by ACS that have projected separations within roughly 30 AU. If these separations are not significantly different from the actual semimajor axis, then many of these binaries should execute one-quarter of an orbit in less than $50 \mathrm{yr}$. While this period is too long for short-term monitoring, near zero inclination systems will already exhibit measurable changes in position angle $\left(\Delta \theta>9^{\circ}\right)$ since they 
were imaged with ACS in 2004 and 2005. Full orbital and stellar parameters for these systems are possible within a human lifetime.

\section{IMPLICATIONS AND DISCUSSION}

Under the assumption that all of the spatially unresolved binaries are in short-period orbits, one can make a crude estimate of the change in orbital energy of the system postmain sequence. For the following, assume a secondary mass of $0.2 M_{\odot}$, a white dwarf mass of $0.6 M_{\odot}$, and a main-sequence progenitor mass of $1.8 M_{\odot}$. If the system avoids a common envelope phase, it expands by a factor of 2.5 (Jeans 1924) following the main sequence, implying initial orbits of $8 \mathrm{AU}$ for companions which are now near $20 \mathrm{AU}$, of which there are several in this study. Applying the same logic to the smallest projected separation of $14 \mathrm{AU}$, it may have been initially as close as 5.5 AU yet effectively avoided drag forces due to the giant envelope.

On the other hand, a typical post-common envelope system has a period near 0.5 days (Morales-Rueda et al. 2005). The change in specific orbital energy between a main-sequence binary (as described above) in a one to few AU orbit, and a post-main-sequence binary in a 0.5 days orbit is essentially independent of the initial semimajor axis for $a_{0}>0.5 \mathrm{AU}$, and is around $3 \times 10^{14} \mathrm{erg} \mathrm{g}^{-1}$. If one then deposits all of this energy into $1.2 M_{\odot}$ of slowly expanding hydrogen atoms during the lifetime of the giant envelope, this imparts roughly $250 \mathrm{~km} \mathrm{~s}^{-1}$ of kinetic energy per atom; sufficient to escape $2.0 M_{\odot}$ from an initial distance of $0.1 \mathrm{AU}$. While this calculation is simple, it demonstrates that the deposition of orbital energy into the envelope is commensurate with the short periods found among white dwarfs with low-mass stellar and substellar companions.

Given this likely trade-off, and all else being equal, one would expect the lowest mass companions to end up in the shortest period orbits and higher mass companions at longer periods. There are likely not enough empirical data to test this in real systems with a continuum of initial orbits, primary and secondary masses. For higher mass secondaries (K dwarfs and brighter) in close orbits, the white dwarf would likely go unrecognized, but this is a very interesting phase space. For example, take the Sirius system: with a semimajor axis of $20 \mathrm{AU}$ and a likely white dwarf progenitor mass of $5.0 \mathrm{M}_{\odot}$, the initial semimajor axis was only $4 \mathrm{AU}$ (Liebert et al. 2005b). The Sirius binary has an orbital eccentricity of 0.6 and the white dwarf progenitor should have directly engulfed Sirius A for a significant portion of its giant evolution, yet did not cause the binary orbit to shrink at all.

Lastly, there may be one example of a white dwarf with a low-mass companion in an orbit that could be described as intermediate. G77-61 is the prototype dwarf carbon star, and Dearborn et al. (1986) reported a radial velocity period of $245 \mathrm{~d}$ due to an unseen companion of $M \sin i \approx 0.55 M_{\odot}$. The mass of this carbon-polluted halo star is uncertain, but its effective temperature suggests a possible analogy with a late $\mathrm{K}$ dwarf or early $M$ dwarf. If the unseen companion is indeed a very cool white dwarf as suggested by the data, then G77-61 is either the longest period system known to emerge from a common envelope or the shortest period system known to have avoided one.

In conclusion, the results of an ACS HRC imaging Snapshot survey reveal a bimodal distribution of orbital separations for low-mass, unevolved companions to white dwarfs. These data support the theoretical picture where low-mass stellar or substel- lar companions spiral inward during a common envelope phase, or migrate outward if the initial orbital separation is sufficient to avoid contact with the asymptotic giant envelope (Nordhaus et al. 2010; Schultz et al. 1996; de Kool \& Ritter 1993). All spatially unresolved binaries in this study are predicted to be in short-period orbits amenable to radial velocity and photometric variability studies. The distribution of $\mathrm{M}$ dwarf companion spectral types argues against any significant mass transfer during the common envelope, indicating the current masses of the secondaries are essentially - to within a few tens of percent or better-the same as their masses at formation.

The authors thank the anonymous referee for a valuable and thorough report that improved the quality of the manuscript, and D. Koester for the use of his white dwarf spectral models. This work is based on observations made with the Hubble Space Telescope which is operated by the Association of Universities for Research in Astronomy under NASA contract NAS 526555. Support for Program number 10255 was provided by NASA through grant HST-GO-10255 from the Space Telescope Science Institute. This publication makes use of data products from the Two Micron All Sky Survey, which is a joint project of the University of Massachusetts and the Infrared Processing and Analysis Center/California Institute of Technology, funded by NASA and the National Science Foundation. This work includes data taken with the NASA Galaxy Evolution Explorer, operated for NASA by the California Institute of Technology under NASA contract NAS5-98034. Some data presented herein are part of the Sloan Digital Sky Survey, which is managed by the Astrophysical Research Consortium for the Participating Institutions (http://www.sdss.org/).

Facility: HST (ACS)

\section{APPENDIX \\ NOTES ON INDIVIDUAL OBJECTS}

$0023+388$. The white dwarf parameters derived by Trembley \& Bergeron (2007) imply a distance of $59 \mathrm{pc}$, and are consistent with the GALEX far- and near-ultraviolet fluxes and $V$-band photometry (McCook \& Sion 2008). This is roughly compatible with the estimated $70 \mathrm{pc}$ distance to the spatially unresolved dM5.5 tertiary, but the dM3 (i.e., $V-K \approx 5.0$ ) secondary star has a photometric distance of only 43 pc. If the 24 ". 6 distant, common proper motion companion is itself a near equal luminosity binary, then all three distance estimates would become amenable. While the widely bound secondary star did not fall within the ACS field of view, this system may have a fourth component.

0034-211. Zuckerman et al. (2003) report $\mathrm{H} \beta$ and $\mathrm{Ca} \mathrm{K}$ line emission in the composite spectrum. The ACS images reveal that the binary is widely separated, and hence for a system without additional components, the emission must originate from activity intrinsic to the $\mathrm{M}$ dwarf.

0116-231. There does not exist a reliable effective temperature estimate for the white dwarf, but it is a soft X-ray source (O'Dwyer et al. 2003) and hotter than DA3 as listed in McCook \& Sion (1999). Wolff et al. (1996) give a lower limit of $24,000 \mathrm{~K}$ based on pointed X-ray observations, and it is bright in both GALEX ultraviolet bandpasses.

0145-221. The ACS photometry of GD 1400 is around 0.25 mag fainter than expected based on a previous analysis (Farihi \& Christopher 2004). Figure 11 plots an 11,500 K DA white dwarf model (Koester 2010) together with GALEX far- and 


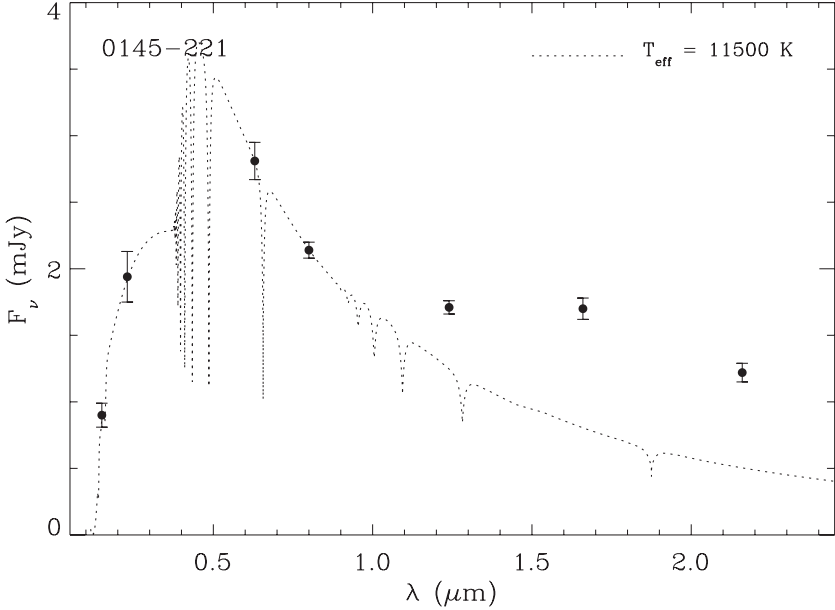

Figure 11. Spectral energy distribution (SED) of GD 1400 overplotted with an $11,500 \mathrm{~K}$ DA model. The photometric data are GALEX far- and near-ultraviolet, optical $r^{\prime}$, ACS (transformed) $I$, and 2MASS $J H K_{s}$. There is no evidence for the L dwarf companion in existing optical data.

near-ultraviolet fluxes, $r^{\prime}$-band and ACS (transformed) $I$-band photometry. Compared to this model and the plotted optical and ultraviolet data, the previously reported $V=14.85 \pm 0.12 \mathrm{mag}$ photometry (Farihi \& Christopher 2004) is too bright. However, GD 1400 is a large-amplitude ZZ Ceti pulsator, varying by up to 0.2 mag with its most significant modes at 462,728 , and $823 \mathrm{~s}$ (Fontaine et al. 2003). The ACS exposures were $1080 \mathrm{~s}$ in total and should represent an average brightness amplitude, while the exposures analyzed by Farihi \& Christopher (2004) were three $60 \mathrm{~s}$ images separated by $90 \mathrm{~s}$. These latter data may have been biased by one or more exposures taken during a bright phase, and combined with their relatively large photometric error, are likely inferior to the ACS photometry. The system is a radial velocity variable with a period of $10 \mathrm{hr}$ (M. R. Burleigh et al. 2010, in preparation).

0208-153. Paper I reported that the PSF width of the M dwarf was not larger than that of the white dwarf, nominally indicating a likely third, cool component broadening the PSF of the hotter star. Re-examination of the older pipeline data (15.4c) confirms that both PSFs have equal widths in those images, while in the subsequent pipeline (15.7) data set the FWHM of the M dwarf is larger by 0.12 pixels, more or less as expected if each star is single.

$0219+282$. This is a triple system; the DAB spectrum of the white dwarf has been shown to be better explained with a composite DA and DB white dwarf (Limoges et al. 2009). The two spatially resolved stars in the ACS image have PSFs indicative of a relatively blue and red object, consistent with a spatially unresolved DA+DB and a visual M dwarf. Radial velocity monitoring of the $\mathrm{DA}$ and $\mathrm{DB}$ components should be able to confirm this scenario.

0257-005. An extreme example of a type 1 (see Section 3.2) photometric distance mismatch, with $\Delta(m-M)=+1.6$ mag. The disparate distance estimates may be reconciled if the $\mathrm{dM}$ is an equal luminosity double and the white dwarf is at the cool, high gravity end allowed by its error bars (Farihi 2009).

$0309-275,0949+451,1001+203,1106+316,1412-049$. These stars have inaccurate coordinates in the literature, SIMBAD, and McCook \& Sion (2008). Accurate ACS positions for the white dwarfs are given in Table 2.

$0324+738$. The white dwarf lies within several arcseconds of two background stars (Paper I) that are brighter at most

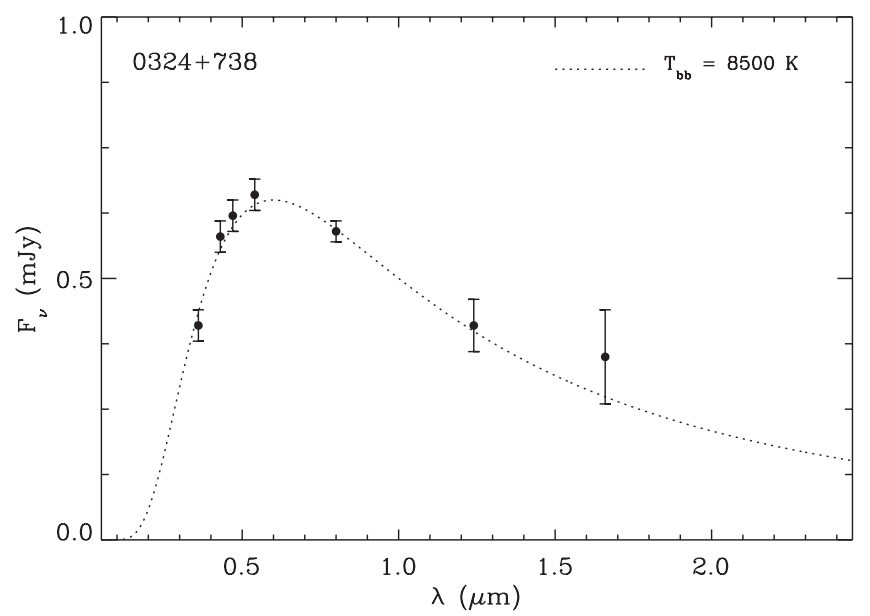

Figure 12. SED of G221-10 overplotted with an $8500 \mathrm{~K}$ blackbody model. The photometric data are short wavelength multichannel measurements from Greenstein (1976), ACS (transformed) $I$, and 2MASS $J H$. The data are consistent with no near-infrared excess.

or all optical wavelengths, resulting in photometric confusion and underestimations of its mass and effective temperature (Bergeron et al. 2001; Greenstein 1984). Perhaps the most reliable archival data are the shorter wavelength multichannel spectrophotometry taken by Greenstein (1976). Combined with the ACS photometry, Figure 12 shows that these data are well fitted by an $8500 \mathrm{~K}$ blackbody model, implying a mass of $0.99 M_{\odot}$ at its $40 \mathrm{pc}$ distance from trigonometric parallax. A near-infrared excess was suspected on the basis of its 2MASS $H=16.18 \pm 0.25 \mathrm{mag}$ but from the plot it is apparent that all the data are consistent with a single star. The common proper motion companion (G221-11) which fell into the ACS field of view by chance was itself spatially resolved into two stars, making the system triple. The two M dwarf components are not spatially resolved in 2MASS, and their photometric deconvolution and subsequent spectral type estimates are somewhat uncertain.

0347-137. This white dwarf has three disparate temperature determinations in the literature (12,600 K, Gianninas et al. 2006; 15,500 K, Zuckerman et al. 2003; 21,300 K, Koester et al. 2001), probably owing to spectroscopic contamination by the $\mathrm{M}$ dwarf secondary. Extrapolating from $U=14.7 \mathrm{mag}$ (Mermilliod 1991) for the white dwarf alone, $\log g=8.0$ models predict $I=15.9 \mathrm{mag}$ for a $21,000 \mathrm{~K}$ star versus $I=15.2 \mathrm{mag}$ for a $12,500 \mathrm{~K}$ star. The ACS photometry yields $I=15.46 \mathrm{mag}$, consistent with $T_{\text {eff }}=15,500 \mathrm{~K}$. The $H S T$ images reveal that the binary is widely separated, and hence for a system without additional components, the Balmer line emission reported by Koester et al. (2001) must originate from activity intrinsic to the $\mathrm{dM}$.

$0354+463$. This DAZ with a late dMe companion is somewhat mysterious as no radial velocity variability searches have been successful yet the composite spectrum has Balmer line emission (Zuckerman et al. 2003; Schultz et al. 1996). The velocities of the $\mathrm{Ca} \mathrm{K}$ absorption in the white dwarf and the Balmer emission lines in the $\mathrm{M}$ dwarf all agree reasonably well $\left(-65 \pm 5 \mathrm{~km} \mathrm{~s}^{-1}\right)$ and collectively represent several measurements over 5 years. Furthermore, the ACS images reveal a single point source, supporting a closely orbiting, post-common envelope binary with $a<1.0 \mathrm{AU}$. Given that the white dwarf atmosphere is polluted with several metals, likely due to a stellar wind from the dM7 companion (Zuckerman et al. 2003), it is virtually certain that the system has a short orbital period. The sum of all the evidence then points to a near pole-on binary with small radial 
velocity changes that may be confirmed through high-resolution spectroscopic monitoring.

0357-233. This system has a severe photometric distance mismatch between its components, $\Delta(m-M)=-1.6$ mag. However, the system is not well studied; Greenstein (1979) remarks that the white dwarf is "very hot" but no spectroscopic temperature estimate exists. The $U B$ photometry reported in Farihi et al. (2005a) is uncontaminated by light from the M dwarf companion, and combined with GALEX fluxes and the ACS I-band photometry for the white dwarf, implies a temperature near 35,000 K. In this particular case, it seems almost certain that the white dwarf luminosity has been significantly underestimated, and must have a large effective radius (a binary or low surface gravity), and probably a higher temperature than estimated here. A good spectroscopic parameter determination for the white dwarf is needed to reconcile the photometric distance discrepancy.

$0430+136$. This system is spatially resolved into two components, both of which are clearly too bright to be a $36,000 \mathrm{~K}$ white dwarf (Trembley \& Bergeron 2007) given the $U B V$ photometry of Wegner et al. (1990). Specifically, such a white dwarf with $U=15.9 \mathrm{mag}$ should have $I=17.6 \mathrm{mag}$ while both ACS imaged components have $I<17.0 \mathrm{mag}$. Assuming the brighter component is an $\mathrm{M}$ dwarf and the dimmer component is an unresolved DA $+\mathrm{dM}$ produces self-consistent results and photometric distances between 100 and $150 \mathrm{pc}$ for the $\mathrm{M}$ dwarf components. There is tentative evidence for a spatially unresolved, tertiary M dwarf; the SPY spectrum of the composite system reveals Balmer emission lines which have shifted between two observations (D. Koester 2010, private communication). While the $\mathrm{M}$ dwarf distance estimates can be brought into agreement if the widely separated component is itself an unresolved double, the photometric distance to the white dwarf is at least $320 \mathrm{pc}$ ( $\log g=8.11$; P. Bergeron 2009, private communication), and is by far the most extreme distance mismatch of the entire sample. The situation can only be amended if the white dwarf has a significantly lower luminosity via high mass, lower temperature, or both.

0458-665. An error in the coordinates and name of RX J0458.9-6628 exists in McCook \& Sion (1999), where it is called 0458-662 based on an incorrect position. Both the position and name have been amended in McCook \& Sion (2008). The ACS image yields precise coordinates for the white dwarf which are listed in Table 2, and nearly identical to the optical discovery coordinates for the binary (Hutchings et al. 1996).

$0518+333$. This star was imaged based on its 2MASS $J H K_{s}$ photometry, which is apparently contaminated by its 7..5 distant $\mathrm{M}$ dwarf secondary. The ACS photometry and the $J H K$ data of Farihi (2009) are consistent with a single white dwarf of $9500 \mathrm{~K}$. However, the proper motion companion was serendipitously resolved into two components, making the system triple.

0752-146. The orbital period of this highly probable close binary has never been established, having fallen just short of strict variability criterion (Maxted et al. 2000). However, three independent radial velocity studies of this system have together seen some variability and large velocity separations between emission and absorption components (Zuckerman et al. 2003; Maxted et al. 2000; Schultz et al. 1996). The ACS image reveals an unresolved point source, making the projected separation of the pair certainly closer than $0.9 \mathrm{AU}$, and the orbit shorter than 1 yr. Further radial velocity monitoring should be able to discern the period and mass ratio of this double-lined spectroscopic binary.
0807+190. LHS 1986 appears to be an erroneous identification by Luyten (1979), as there is no cataloged optical or near-infrared (e.g., USNO, 2MASS) point source located within $10^{\prime \prime}$ of the position $08^{\mathrm{h}} 10^{\mathrm{m}} 02.90^{\mathrm{s}}+18^{\circ} 52^{\prime} 09^{\prime \prime}$. (J2000; Bakos et al. 2002). The only object with significant proper motion within $1^{\prime}$ of these coordinates moves at $0^{\prime \prime} 06 \mathrm{yr}^{-1}$ at position $08^{\mathrm{s}} 10^{\mathrm{s}} 00.29^{\mathrm{s}}+18^{\circ} 51^{\prime} 46^{\prime \prime} 6$ (J2000; Zacharias et al. 2005); this is the target observed by ACS. There is no entry for LHS 1986 in the revised NLTT catalog (Salim \& Gould 2003), nor the LSPM catalog, confirming the likely error (S. Lepine 2009, private communication; Lépine \& Shara 2005). Furthermore, the ACS imaged star is not a white dwarf; there is no detection in GALEX and it is an SDSS and 2MASS stellar source with ugrizJHK photometry consistent with a mid to late M dwarf at $d \gtrsim 200 \mathrm{pc}$ (Bochanski et al. 2007; Kirkpatrick \& McCarthy 1994), consistent with its modest proper motion. The analysis by Gizis \& Reid (1997) and the optical spectrum shown in Reid \& Gizis (2005) - assuming this is the same object as in the ACS images-both indicate a K-type star. Gizis \& Reid (1997) remark the star may have been misidentified, and the original LHS catalog gives a color type of "k" (Luyten 1979). This star is likely a relatively fast moving but a distant, metal-poor sdK star with reddened optical colors, whose proper motion was misjudged.

$0824+288$. This spectroscopic composite $\mathrm{DA}+\mathrm{dC}(\mathrm{e})$ binary has been resolved into two closely separated components at 0'.077, plus the wider tertiary M dwarf companion at 3.'33. While it is not certain if the two closest, spatially resolved components are the $\mathrm{dC}$ and DA stars (each one could be an unresolved binary, and the other a fourth component), this is the most likely explanation and most consistent with all the photometry (Farihi et al. 2005a), and the shape and width of the deconvolved ACS PSFs. At the adopted photometric distance to the system, the DA+dC pair is currently separated by at least $14 \mathrm{AU}$, challenging the scenario in which a $\mathrm{dC}$ star forms via mass transfer (Heber et al. 1993).

0908+226. This star (LP 369-15) is listed as a part of a common proper motion pair with LP 369-14 at a separation of $200^{\prime \prime}$ and position angle $277^{\circ}$, both stars moving at $0^{\prime} \cdot 11 \mathrm{yr}^{-1}$ along $120^{\circ}$ (Silvestri et al. 2005; Luyten 1997). This appears to be a spurious entry in the LDS catalog as there are no such objects within a few arcminutes of LP 369-15, and no entries in the revised NLTT or the LSPM catalogs (Lépine \& Shara 2005; Salim \& Gould 2003). Its proper motion is given as zero in both the USNO and NOMAD catalogs (Zacharias et al. 2005; Monet et al. 2003), and blinking the POSS I and II plate scans verifies the star moves little or not at all in 45 years. Also, the coordinates of LP 369-15 in SIMBAD and the Luyten catalogs are inaccurate by more than a dozen arcseconds (Table 2 lists its ACS imaged position). Although its proper motion was apparently misjudged by Luyten (1997), its inclusion in a list of white dwarfs appears to be correct; SDSS data reveal the star (J091143.09+222748.8) to be a DA+dMe spectroscopic composite, with proper motion $0^{\prime \prime} 005 \mathrm{yr}^{-1}$.

0933+025, 1033+464. Schultz et al. (1996) notes $\mathrm{H} \alpha$ emission in the composite spectra of these binaries, which must be attributed to an active red dwarf since the systems are spatially resolved and hence widely separated.

0937-095. The ACS field of view captured both components of the common proper motion binary LDS 3913, separated by $13^{\prime \prime} .54$ at position angle $354.8^{\circ}$ and comoving at $(99,-236)$ mas yr ${ }^{-1}$ (Salim \& Gould 2003; Luyten 1997). The primary, LDS 3913A (also LP 668-9, G161-56, NLTT 22302), is almost certainly a K-type star, while the secondary, LDS 3913B (also, 


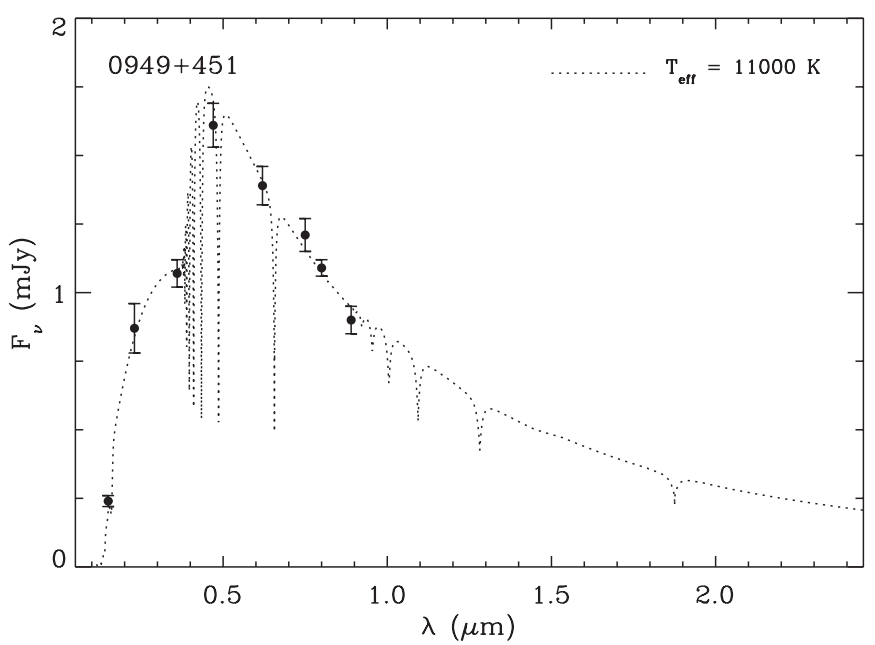

Figure 13. SED of HS 0949+4508 overplotted with an 11,000 K DA model. The photometric data are GALEX far- and near-ultraviolet, SDSS ugriz, and ACS (transformed) I.

LP 668-10, NLTT 22301), also appears to be a late $\mathrm{K}$ or early $\mathrm{M}$ dwarf. This conclusion is supported by $U B V$ (Silvestri et al. 2005; Salim \& Gould 2003; Mermilliod 1991), ACS $I$-band, and 2MASS $J H K_{s}$ (Skrutskie et al. 2006) photometry, which yield $V-K=2.9$ and $V-K=3.4-$ consistent with spectral types K5 and M0-for LDS 3193A and B, respectively. Two main-sequence stars of corresponding spectral type would lie at $d \approx 500 \mathrm{pc}$, and with tangential speeds of $v \approx 600 \mathrm{~km} \mathrm{~s}^{-1}$. Such high velocities suggest kinematic membership in the galactic thick disk or halo, and hence these cool stars are probably metal-poor subdwarfs. Assuming the pair lie 1 mag below the main sequence, their distance would be $d \approx 340 \mathrm{pc}$ with tangential speeds of $v \approx 400 \mathrm{~km} \mathrm{~s}^{-1}$, and $(U, V, W)=(-340,-210,-100) \mathrm{km} \mathrm{s}^{-1}$. Therefore, in all likelihood, this pair belongs to the thick disk or halo.

0949+451. This system is not well studied and Figure 13 plots GALEX, SDSS, and the ACS I-band photometry for the white dwarf, fitted with an 11,000 K DA white dwarf model. Although the ugriz photometry is somewhat uncertain due to the close visual $\mathrm{M}$ dwarf companions, the data are altogether well modeled. At a photometric distance of $65 \mathrm{pc}$, the double $\mathrm{M}$ dwarf has a projected separation of only $2.5 \mathrm{AU}$.

1015-173. This pair has an extreme photometric distance mismatch between its components. The system is not well studied, but GALEX and $U B V$ photometry (Kilkenny et al. 1997) support a relatively hot white dwarf. Still, the 25,000 K estimate found here produces a distance far too close to match the $\mathrm{M}$ dwarf distance estimate; even an equally luminous double white dwarf is insufficient to bridge the gap. Therefore, the white dwarf is likely to be intrinsically brighter via temperature or radius.

1036-204. This white dwarf has a peculiar SED described by very different temperatures in the ultraviolet-blue versus the optical red and near-infrared (Farihi 2009) The broad and deep optical $\mathrm{C}_{2}$ absorption bands are likely responsible for a significant increase in its near-infrared fluxes via the redistribution of emergent energy; there is no evidence for a companion in this system.

1051+516. Silvestri et al. (2006) derive $T_{\text {eff }}=29,600 \mathrm{~K}$, $\log g=8.1$ for this star based on the SDSS data, but these values are likely to be uncertain owing to contamination from the bright $\mathrm{M}$ dwarf companion The GALEX fluxes for the white dwarf favor the lower effective temperature estimate used here, which leads to better agreement in the component distances.

1106-211. There is no spectroscopic, photometric, nor kinematical evidence that this star is a white dwarf (McCook \& Sion 2008). As Hoard et al. (2007) note correctly, there is no star with high proper motion within several arcminutes of the coordinates given in Evans (1992). The object located at those coordinates has a proper motion of $0.03 \mathrm{yr}^{-1}$ (Zacharias et al. 2005), and optical through near-infrared colors consistent with a late $\mathrm{K}$ dwarf (e.g., $V-K=3.3$ ).

$1108+325$. Component identification in this system was not clear-cut. The deconvolved FWHMs are nearly identical, as are the instrumental magnitudes prior to color correction, although the former were certainly affected by close binarity. The northwest and southeast objects were identified as the cool companion and white dwarf, respectively. This choice fits the expected brightness of the white dwarf at $I$ band, based on its GALEX and SDSS $u g$ photometry, whereas if the roles are reversed, the photometric distances become more discrepant. Additionally, the best daophot PSF fits for the northwest and southeast source were found using $\mathrm{M}$ dwarf and white dwarf templates, respectively. The $J H K$ photometry for this system was taken from Farihi (2009) as the 2MASS data have low S/N at the two longer wavelengths.

$1133+358$. This rare DC+dM binary is unresolved in the ACS images, implying a projected separation under $1.3 \mathrm{AU}$ at its trigonometric parallax distance of $50 \mathrm{pc}$ (Dahn et al. 1988). The spectrum in Putney (1997) shows several Balmer emission lines from an active and optically dominant $\mathrm{M}$ dwarf. Without such emission potentially masking metal absorption in the white dwarf, one might expect the cool helium atmosphere star to be readily polluted by the wind from the secondary (Zuckerman et al. 2003). The star is detected in the GALEX near-ultraviolet bandpass, and combined with ground-based ultraviolet photometry, confirms the presence of a $6500 \mathrm{~K}$ white dwarf (Greenstein 1976). For this temperature, the parallax distance yields $M_{V}=13.37$ mag and a white dwarf mass of $0.41 M_{\odot}$, suggesting that the $\mathrm{M}$ dwarf companion is in a close orbit and ejected sufficient mass during the first ascent giant phase to prevent helium fusion.

1156+132. Unfortunately, inaccurate coordinates for this star in McCook \& Sion $(2008,1999)$ caused the white dwarf to fall outside of the $25^{\prime \prime} \times 25^{\prime \prime}$ field of view of the ACS HRC; hence no data were obtained for this star. Table 2 lists both the incorrect and correct positions for the star, and the error has been corrected in SIMBAD and the current online white dwarf catalog.

$1156+129$. This white dwarf is poorly studied, and the effective temperature estimate made here relies on the SDSS $u$-band and GALEX fluxes, but is still relatively uncertain.

1236-004. The white dwarf and $\mathrm{M}$ dwarf components of this binary were misidentified in Paper I. The ACS stellar profiles suggest that the western component is the M dwarf (with a larger FWHM), and the SDSS color composite finding chart reveals a clear change in the halo of the stellar image from blue in the east, to white-orange in the west. The newer analysis presented here should be considered a correction to Paper I.

$1247+550$. This cool white dwarf has published $J H K$ photometry (Bergeron et al. 2001) which differs significantly from the relatively low $\mathrm{S} / \mathrm{N}$ photometry of $2 \mathrm{MASS}$; these superior quality data suggest that the star does not have a near-infrared excess. 


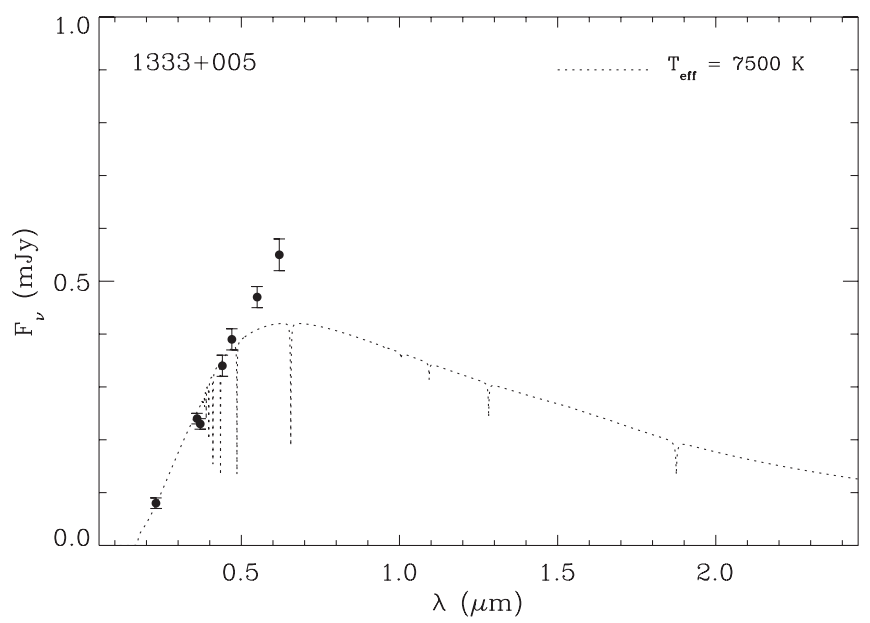

Figure 14. SED of LP 618-14 overplotted with an $7500 \mathrm{~K}$ DA model. The photometric data are GALEX near-ultraviolet, SDSS $u g r$, and optical $U B V$ (Farihi et al. 2005a). The photometry redward of $5000 \AA$ was ignored in the model fit due to the influence of the M dwarf; these longer wavelength fluxes are all above $1.0 \mathrm{mJy}$.

1333+487. Surprisingly, the HST ACS observations are the first published data to establish the spatially resolved, visual binary nature of GD 325, at angular separation 2".95 and position angle 72.1 (Paper I; Greenstein 1974). The USNO-B1 optical and 2MASS near-infrared catalog positions for the source are offset by $2^{\prime \prime} .3$ at $76^{\circ}$. Based on $U B V$ data (McCook \& Sion 1999), the ACS photometry of the spatially resolved white dwarf is too bright for a single star of 16,000 K (Castanheira et al. 2006). Furthermore, the FWHM of the white dwarf is wider than that of the spatially resolved M dwarf, a singular example among the 29 well-resolved pairs (GD 325 is a clear outlier in Figure 2). Assigning the photometric excess and wide PSF to a spatially unresolved tertiary produces self-consistent results and distance estimates of 46 and $51 \mathrm{pc}$ to the $\mathrm{M}$ dwarf components. However, the white dwarf has a trigonometric parallax distance of $34 \mathrm{pc}$ (Dahn et al. 1982; McCook \& Sion 1999), but it is possible that the astrometry was biased due to binarity; there is no mention of the visual companion in the literature.

$1333+005$. There is an extreme distance discrepancy between the components in this spatially unresolved binary, strongly suggesting that the white dwarf is a low-mass, helium core degenerate. GALEX near-ultraviolet and short wavelength ground-based photometry suggest that the white dwarf is warmer than previous spectroscopic estimates (Kilic et al. 2006), likely influenced by the $\mathrm{M}$ dwarf continuum flux. Figure 14 shows that a $7500 \mathrm{~K}$ DA model yields a good fit to the ultraviolet through blue photometry. At the photometric distance to the M dwarf, the white dwarf would have $\log g<7.0$ and a mass below $0.2 M_{\odot}$. Such an extreme solution indicates that the secondary is likely to be subluminous relative to the $\mathbf{M}$ dwarf templates used here. In any event, the white dwarf is likely to have a low mass and a commensurately large radius.

1339+346. The optical companion in the ACS images appears to be a background early K-type star; a result of chance alignment and not physical. The cool star imaged with ACS is part of a wide, common proper motion pair of $\mathrm{K}$ stars with $0.05 \mathrm{yr}^{-1}$ (Zacharias et al. 2005); the $\mathrm{K}$ star nearest to the white dwarf is $2^{\prime \prime} .5$ distant at $213^{\circ}$, while the second $\mathrm{K}$ star is offset by $21^{\prime \prime} .2$ at $298^{\circ}$. The two cool stars have ugrizJHK photometry indicating approximate spectral types of $\mathrm{K} 2$ and K5 at around 720 pc (Farihi 2009; Abazajian et al. 2009), while the white dwarf distance is near 100 pc (Liebert et al. 2005a). Examination of photographic plate scans taken more than 55 years ago reveals the white dwarf has moved relative to the nearest $\mathrm{K}$ star, confirming they are not physically associated (Farihi 2009).

1412-049. There are virtually no constraints on the properties of this white dwarf; Table 3 lists a best guess based on $G A L E X$ fluxes and constraints from the red dwarf spectral type estimate.

1419+576. The white dwarf does not have any published parameters, and the SDSS spectrum shows the $M$ dwarfs dominating the emergent flux beginning at $5000 \AA$. The GALEX, $u$-band, and ACS (transformed) I-band photometry support a rough effective temperature estimate of $25,000 \mathrm{~K}$. For a reasonable range of photometric distances, the visual double $\mathrm{M}$ dwarf has a projected separation less than $11 \mathrm{AU}$.

$1433+538$. This spatially unresolved binary is somewhat mysterious. Its spectrum contains no obvious emission lines expected in a close binary; the $\mathrm{H} \alpha$ region was flat during four temporally separate observations (Schultz et al. 1996), while the SDSS spectrum shows well-defined $\mathrm{H} \alpha$ absorption (Silvestri et al. 2006). Close inspection of the unbinned SDSS data reveal possible weak $\mathrm{H} \alpha$ and $\mathrm{Na} 8190 \AA$ emission features, but these may be due to noise. There are two published trigonometric parallaxes for this point source, both of which are smaller than the quoted measurement error (McCook \& Sion 1999; Dahn et al. 1988), and hence neither of these distances are used.

1434+289. A near-infrared spectrum of this white dwarf reveals no excess emission; the 2MASS data have low $\mathrm{S} / \mathrm{N}$ at $H K_{s}$ (Farihi 2009).

1436-216. This system is a spatially unresolved DA+dMe recently discovered by Zuckerman et al. (2003). There are few constraints on the white dwarf parameters, and those used here are a best guess based on optical $U B V$ and GALEX fluxes.

1458+171. Liebert et al. (2005a) derive parameters that indicate this as a low-mass, helium core white dwarf, consistent with a short-period binary.

1558+616. The parameters of this white dwarf are unconstrained in the literature (McCook \& Sion 2008). It has no GALEX data, but the SDSS $u-g$ color suggests an effective temperature near $15,000 \mathrm{~K}$, assuming little or no contamination in the $g$ band by the M dwarf.

$1603+125$. This star is not a white dwarf; the original designation (McCook \& Sion 1999) was likely intended to be $1603+175$ which has a corresponding source in the KUV catalog (P. Bergeron 2008, private communication); and has been changed accordingly in McCook \& Sion (2008). The source imaged by ACS is likely a K star based on its ugrizJHK photometry and small proper motion (Abazajian et al. 2009; Zacharias et al. 2005).

$1619+525$. This possible triple system is discussed in detail by Farihi (2009) and in Paper I. A bound triple is possible at a shared distance near $290 \mathrm{pc}$; the photometric distance to both spatially resolved $\mathrm{M}$ dwarf components. However, this is significantly further than the $100 \mathrm{pc}$ distance estimate to the white dwarf from Balmer line spectroscopy (Liebert et al. 2005a). A chance alignment is highly improbable, and no relative motion is seen between any of the three stars. While the physical association of these three stars remains tentative, the white dwarf parameters may be skewed due to spectroscopic contamination.

$1631+781$. This triple system is discussed in detail in Paper I. Here, owing to recently published white dwarf parameters (Trembley \& Bergeron 2007) and the newer ACS analysis 
which photometrically deconvolves the two M dwarfs, excellent agreement is now found between the component photometric distances. The $\mathrm{M}$ dwarfs are widely separated from the white dwarf yet show clear signs of activity via Balmer line emission variability in strength but not velocity (Sion et al. 1995). Hence, it is possible that the observed EUV and variable X-ray emission may originate from a pair of coronally active $M$ dwarfs, rather than the white dwarf as previously reported (Cooke et al. 1992).

$1658+440$. The apparent $2 \mathrm{MASS} K_{s}$-band excess of this massive, magnetic white dwarf is spurious; Spitzer IRAC data reveal no infrared excess (Farihi et al. 2008).

1717-345. This system has uncertain white dwarf parameters in the literature, as the optical spectrum is dominated by the red dwarf (Kawka et al. 2004; Reid et al. 1988). Additionally, there is a lack of GALEX or ground-based photoelectric ultraviolet or blue photometry which might provide better constraints.

$1833+644$. This is a likely triple system, although the candidate tertiary detected at $1^{\prime \prime} .8$ has yet to be confirmed with additional observations. The evidence in favor of a bound system is (1) the modest galactic latitude $b=+26^{\circ}$ at $\ell=94^{\circ}$ (normal to the direction toward the galactic center), (2) the angular separation, and (3) the very wide FWHM expected for a late $\mathrm{M}$ dwarf. The probability of a late $\mathrm{M}$ dwarf being found within a cylinder centered at the white dwarf, of radius $2^{\prime \prime}$ and length $100 \mathrm{pc}$ is $5 \times 10^{-6}$ (Cruz et al. 2007).

$1845+683,2211+372,2323+256,2349-283$. These are not binary suspects (Farihi 2009; Paper I).

2151-015. Paper I suggested the white dwarf PSF appeared too red for a single star, possibly indicating a third component. The position of the white dwarf in Figure 2 firmly discounts this possibility. The ACS observations confirm the suspicions of both Zuckerman et al. (2003) and Maxted \& Marsh (1999) that this system is not a close binary.

2237-365. This white dwarf-M dwarf system is correctly designated HE 2237-3630 but is not the same star as LHS 3841. This unfortunate co-identification was made in Friedrich et al. (2000) who took a spectrum of the DC+dM binary and who list the correct position for the white dwarf system. SuperCOSMOS images reveal a close visual white dwarf-M dwarf pair, solidified by GALEX and 2MASS photometry. Believing the two designations corresponded to the same source, the ACS observations targeted LHS 3841, a metal-poor halo star (Reid \& Gizis 2005), and hence no data were obtained for the white dwarf binary system. Table 2 lists the positions of both LHS 3841 and HE 2237-3630.

$2257+162$. The white dwarf is most likely a low-mass, helium core degenerate (Liebert et al. 2005a) and the system is almost certainly a short-period binary. Neither Liebert et al. (2005a) nor Trembley \& Bergeron (2007) report the detection of the red dwarf in their optical spectra.

2336-187. This appears to be a single white dwarf without any $K$-band excess; the 2MASS flux is $2 \sigma$ brighter than that measured independently by Farihi (2009). The resulting $I-K$ color is consistent with a $8100 \mathrm{~K} \mathrm{DA}$ white dwarf photosphere.

\section{REFERENCES}

Abazajian, K. N., et al. 2009, ApJS, 182, 543

Bakos, G. A., Sahu, K. C., \& Nemeth, P. 2002, ApJS, 141, 187

Bergeron, P., Leggett, S. K., \& Ruiz, M. T. 2001, ApJS, 133, 413

Bergeron, P., Saffer, R. A., \& Liebert, J. 1992, ApJ, 394, 228

Bergeron, P., Saumon, D., \& Wesemael, F. 1995a, ApJ, 443, 764

Bergeron, P., Wesemael, F., \& Beauchamp, A. 1995b, PASP, 107, 1047

Bochanski, J. J., West, A. A., Hawley, S. L., \& Covey, K. R. 2007, AJ, 133, 531
Bohlin, R. C. 2007, in ASP Conf. Ser. 364, The Future of Photometric, Spectrophotometric and Polarimetric Standardization, ed. C. Sterken (San Francisco, CA: ASP), 315

Burleigh, M. R., Clarke, F. J., \& Hodgkin, S. T. 2002, MNRAS, 331, L41

Castanheira, B. G., Kepler, S. O., Handler, G., \& Koester, D. 2006, A\&A, 450, 331

Cooke, B. A., et al. 1992, Nature, 335, 61

Copenhagen University Observatory 2006, The Carlsberg Meridian Catalog 14 (Strasbourg: CDS)

Cruz, K. L., Reid, I. N., Liebert, J., Kirkpatrick, J. D., \& Lowrance, P. J. 2003, AJ, 126,2421

Cruz, K. L., et al. 2007, AJ, 133, 439

Dahn, C. C., Liebert, J., Kron, R. G., Spinrad, H., \& Hintzen, P. M. 1977, ApJ, 216,757

Dahn, C. C., et al. 1982, AJ, 87, 419

Dahn, C. C., et al. 1988, AJ, 95, 237

Dahn, C. C., et al. 2002, AJ, 124, 1170

Dearborn, D. S. P., Liebert, J., Aaronson, M., Dahn, C. C., Harrington, R., Mould, J., \& Greenstein, J. L. 1986, ApJ, 300, 314

Debes, J. H. 2006, ApJ, 652, 636

de Kool, M., \& Ritter, H. 1993, A\&A, 267, 397

De Marco, O. 2009, PASP, 121, 316

DENIS Consortium 2005, The DENIS Database, 3rd Release (Strasbourg: CDS)

Dobbie, P. D., Burleigh, M. R., Levan, A. J., Barstow, M. A., Napiwotzki, R., Holberg, J. B., Hubeny, I., \& Howell, S. B. 2005, MNRAS, 357, 1049

Dobbie, P. D., Napiwotzki, R., Burleigh, M. R., Williams, K. A., Sharp, R., Barstow, M. A., Casewell, S. L., \& Hubeny, I. 2009, MNRAS, 395, 2248

Dreizler, S., \& Werner, K. 1996, A\&A, 314, 217

Eggen, O. J., \& Greenstein, J. L. 1965, ApJ, 142, 925

Evans, D. W. 1992, MNRAS, 255, 521

Farihi, J. 2004, PhD thesis, UCLA

Farihi, J. 2009, MNRAS, 398, 2091

Farihi, J., Becklin, E. E., \& Zuckerman, B. 2005a, ApJS, 161, 394

Farihi, J., \& Christopher, M. 2004, AJ, 128, 1868

Farihi, J., Hoard, D. W., \& Wachter, S. 2006, ApJ, 646, 480

Farihi, J., Jura, M., \& Zuckerman, B. 2009, ApJ, 694, 805

Farihi, J., Zuckerman, B., \& Becklin, E. E. 2005b, AJ, 130, 2237

Farihi, J., Zuckerman, B., \& Becklin, E. E. 2008, ApJ, 674, 431

Finley, D. S., Koester, D., \& Basri, G. 1997, ApJ, 488, 375

Fleming, T. A., Snowden, S. L., Pfefferman, E., Briel, U., \& Greiner, J. 1996, A\&A, 316, 147

Fontaine, G., Bergeron, P., Billères, M., \& Charpinet, S. 2003, ApJ, 591, 1184

Fontaine, G., Brassard, P., \& Bergeron, P. 2001, PASP, 113, 409

Ford, H. C., et al. 1998, Proc. SPIE, 3356, 234

Friedrich, S., Koester, D., Christlieb, N., Reimers, D., \& Wisotzki, L. 2000, A\&A, 363, 1040

Gianninas, A., Bergeron, P., \& Fontaine, G. 2006, AJ, 132, 831

Gizis, J. E., \& Reid, I. N. 1997, PASP, 109, 849

Green, P. J., Ali, B., \& Napiwotzki, R. 2000, ApJ, 540, 992

Green, R. F., Schmidt, M., \& Liebert, J. 1986, ApJS, 61, 305

Greenstein, J. L. 1974, AJ, 79, 964

Greenstein, J. L. 1976, ApJ, 207, L119

Greenstein, J. L. 1979, ApJ, 227, 224

Greenstein, J. L. 1984, ApJ, 276, 602

Heber, U., Bade, N., Jordan, S., \& Voges, W. 1993, A\&A, 267, L31

Hintzen, P. 1986, AJ, 92, 431

Hoard, D. W., Wachter, S., Sturch, L. K., Widhalm, A. M., Weiler, K. P., Pretorius, M. L., Wellhouse, J. W., \& Gibiansky, M. 2007, AJ, 134, 26

Hogan, E., Burleigh, M. R., \& Clarke, F. J. 2009, MNRAS, 396, 2074

Holberg, J. B., \& Bergeron, P. 2006, AJ, 132, 1221

Holman, M. J., \& Wiegiert, P. A. 1999, AJ, 117, 621

Hügelmeyer, S. D., Dreizler, S., Homeier, D., Krzesiński, J., Werner, K., Nitta, A., \& Kleinman, S. J. 2006, A\&A, 454, 617

Hutchings, J. B., Crampton, D., Cowley, A. P., Schmidtke, P. C., McGrath, T. K., \& Chu, Y. H. 1995, PASP, 107, 931

Jeans, J. H. 1924, MNRAS, 85, 2

Kawka, A., Vennes, S., \& Thorstensen, J. R. 2004, AJ, 127, 1702

Kilic, M., et al. 2006, AJ, 131, 582

Kilkenny, D., O’Donoghue, D., Koen, C., Stobie, R. S., \& Chen, A. 1997, MNRAS, 287, 867

Kirkpatrick, J. D., \& McCarthy, D. W. 1994, AJ, 107, 333

Koester, D. 2009, A\&A, 498, 517

Koester, D. 2010, Mem. Soc. Astron. Ital., in press (arXiv:0812.0482)

Koester, D., et al. 2001, A\&A, 378, 556

Lamontagne, R., Demers, S., Wesemael, F., Fontaine, G., \& Irwin, M. J. 2000, AJ, 119, 241 
Leggett, S. K. 1992, ApJS, 82, 351

Lépine, S., \& Shara, M. 2005, AJ, 129, 1483

Liebert, J., Bergeron, P., \& Holberg, J. B. 2003, AJ, 125, 348

Liebert, J., Bergeron, P., \& Holberg, J. B. 2005a, ApJS, 156, 47

Liebert, J., Schmidt, G. D., Lesser, M., Stepanian, J. A., Lipovetsky, V. A., Chaffe, F. H., Foltz, C. B., \& Bergeron, P. 1994, ApJ, 421, 733

Liebert, J., Schmidt, G. D., Sion, E. M., Starrfield, S. G., Green, R. F., \& Boroson, T. A. 1985, PASP, 97,158

Liebert, J., Young, P. A., Arnett, D., Holberg, J. B., \& Williams, K. A. 2005b, ApJ, 630, L69

Limoges, M. M., Bergeron, P., \& Dufour, P. 2009, ApJ, 696, 1461

Luyten, W. J. 1979, The LHS Catalog (Minneapolis, MN: Univ. Minnesota)

Luyten, W. J. 1997, The LDS Catalogue (Minneapolis, MN: Univ. Minnesota)

Martin, D. C., et al. 2005, ApJ, 619, L1

Maxted, P. F. L., \& Marsh, T. R. 1999, MNRAS, 307, 122

Maxted, P. F. L., Marsh, T. R., \& Moran, C. K. J. 2000, MNRAS, 319, 305

McCook, G. P., \& Sion, E. M. 1999, ApJS, 121, 1

McCook, G. P., \& Sion, E. M. 2008, Catalog of Spectroscopically Identified White Dwarfs (Strasbourg: CDS)

Mermilliod, J. C. 1991, Homogeneous Means in the UBV System (Strasbourg: CDS)

Monet, D., et al. 2003, AJ, 125, 984

Morales-Rueda, L., Marsh, T. R., Maxted, P. F. L., Nelemans, G., Karl, C., Napiwotzki, R., \& Moran, C. K. J. 2005, MNRAS, 359, 648

Mullally, F., Kilic, M., Reach, W. T., Kuchner, M. J., von Hippel, T., Burrows, A., \& Winget, D. E. 2007, ApJS, 171, 206

Nelemans, G., et al. 2005, A\&A, 440, 1087

Nordhaus, J., Spiegel, D. S., Ibgui, L., Goodman, J., \& Burrows, A. 2010, MNRAS, in press (arXiv:1002.2216)

O’Dwyer, I. J., Chu, Y. H., Gruendel, R. A., Guerrero, M. A., \& Webbink, R. F. 2003, AJ, 125, 2239

Oswalt, T. D., Foltz, C. B., \& Peterson, B. M. 1984, AJ, 89, 421

Paczynski, B. 1976, in Proc. IAU Symp. 73, Structure and Evolution of Close Binary Systems, ed. P. Eggleton, S. Mitton, \& J. Whelan (Dordrecht: D. Reidel), 75

Probst, R. 1983, ApJS, 53, 335

Putney, A. 1997, ApJS, 112, 527
Raymond, S. N., et al. 2003, AJ, 125, 2621

Rebassa-Mansergas, A., Gänsicke, B. T., Rodríguez-Gil, P., Schreiber, M. R., \& Koester, D. 2007, MNRAS, 382, 1377

Rebassa-Mansergas, A., et al. 2008, MNRAS, 390, 1635

Reid, I. N. 1996, AJ, 111, 2000

Reid, I. N., \& Gizis, J. E. 2005, PASP, 117, 676

Reid, I. N., Wegner, G., Wickramasinghe, D. T., \& Bessell, M. S. 1988, AJ, 96, 275

Saffer, R. A., Wade, R. A., Liebert, J., Green, R. F., Sion, E. M., Bechtold, J., Foss, D., \& Kidder, K. 1993, AJ, 105, 1945

Salim, S., \& Gould, A. 2003, ApJ, 582, 1011

Schreiber, M. R., et al. 2010, A\&A, 513, L7

Schultz, G., Zuckerman, B., \& Becklin, E. E. 1996, ApJ, 460, 402

Silvestri, N. M., Hawley, S. L., \& Oswalt, T. D. 2005, AJ, 129, 2428

Silvestri, N. M., et al. 2006, AJ, 131, 1674

Sion, E. M., Holberg, J. B., Barstow, M. A., \& Kidder, K. M. 1995, PASP, 107, 232

Sirianni, M., et al. 2005, PASP, 117, 1049

Skrutskie, M. F., et al. 2006, AJ, 131, 1163

Stepanian, J. A., Green, R. F., Foltz, C. B., Chaffee, F., Chavushyan, V. H., Lipovetsky, V. A., \& Erastova, L. K. 2001, AJ, 122, 3361

Tappert, C., Gänsicke, B. T., Zorotovic, M., Toledo, I., Southworth, J., Papadaki, C., \& Mennickent, R. E. 2009, A\&A, 504, 491

Tremblay, P. E., \& Bergeron, P. 2007, ApJ, 657, 1013

Vennes, S., Thejll, P. A., Galvan, R. G., \& Dupuis, J. 1997, ApJ, 480, 714

Wachter, S., Hoard, D. W., Hansen, K. H., Wilcox, R. E., Taylor, H. M., \& Finkelstein, S. L. 2003, ApJ, 586, 1356

Wagner, R. M., Sion, E. M., Liebert, J., Starrfield, S. G., \& Zotov, N. 1986, PASP, 98,552

Wegner, G., Africano, J. L., \& Boodrich, B. 1990, AJ, 99, 1907

Wegner, G., McMahan, R. K., \& Boley, F. I. 1987, AJ, 94, 1271

Wolff, B., Jordan, S., \& Koester, D. 1996, A\&A, 307, 149

Zacharias, N., Monet, D. G., Levine, S. E., Urban, S. E., Gaume, R., \& Wycoff, G. L. 2005, The NOMAD Catalog (Strasbourg: CDS)

Zuckerman, B., \& Becklin, E. E. 1992, ApJ, 386, 260

Zuckerman, B., Koester, D., Reid, I. N., \& Hünsch, M. 2003, ApJ, 596, 477 